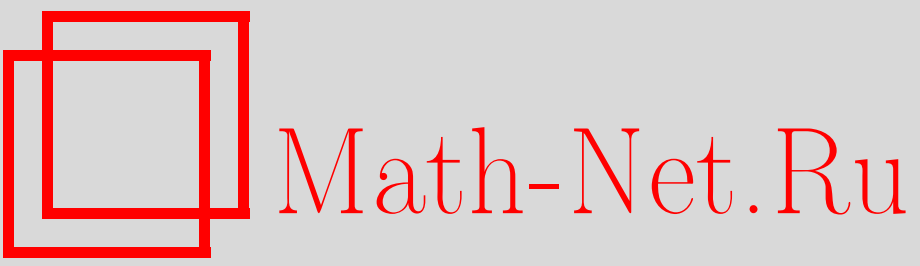

М. Э. Казарян, Мультиособенности, кобордизмы и исчислительная геометрия, УМН, 2003, том 58, выпуск 4, 29-88

DOI: https://doi.org/10.4213/rm642

Использование Общероссийского математического портала Math-Net.Ru подразумевает, что вы прочитали и согласны с пользовательским соглашением

http://www.mathnet.ru/rus/agreement

Параметры загрузки:

IP: 54.224 .60 .19

26 апреля 2023 г., 06:21:08 


\title{
МУЛЬТИОСОБЕННОСТИ, КОБОРДИЗМЫ И ИСЧИСЛИТЕЛЬНАЯ ГЕОМЕТРИЯ
}

\author{
М.Э. КАЗАРЯН
}

\begin{abstract}
В статье приводится универсальная формула для характеристических классов, двойственных циклам мультиособенностей голоморфных отображений, через так называемые остаточные многочлены. Теорема о существовании такой универсальной формулы обобщает на случай мультиособенностей теорему о существовании многочлена Тома. Приведен аналог этой формулы для случая лехандровых особенностей. Вычислены остаточные многочлены особенностей невысоких коразмерностей. Приложения представленной формулы дают, в частности, обобщения на случай $n>3$ классических результатов Плюккера и Сальмона об исчислении особенностей касания гладкой гиперповерхности в $\mathbb{C} P^{n}$ с проективными подпространствами.

Библиография: 56 названий.
\end{abstract}

\section{СОДЕРЖАНИЕ}

Введение

1. Многочлены Тома локальных особенностей . . . . . . . . . . . . . . . . . . . 36

2. Формулировка основной теоремы для мультиособенностей ... . . . . . 39

3. Уточненная формулировка и остаточные многочлены для мультиосо-

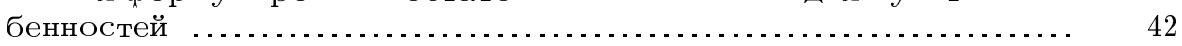

4. Пример: формула кратных особенностей ... . . . . . . . . . . . . . . . . 46

5. Определение остаточных многочленов $R_{\alpha} \ldots \ldots \ldots \ldots \ldots \ldots \ldots \ldots \ldots$

6. Обоснование из теории кобордизмов .............................. . . . . 58

7. Целочисленная формула и другие обобщения . . . . . . . . . . . . . . . . . . 66

8. Изолированные особенности гиперповерхностей и лежандровы характеристические классы ......................... 71

9. Вычисление лежандровых остаточных классов ............... 75

10. Приложения к исчислительной геометрии $\ldots \ldots \ldots \ldots \ldots \ldots \ldots \ldots$

11. Заключительные замечания ................................ . . . . . 85

Список литературы ......................................... 86

Работа выполнена при частичной поддержке грантов INTAS № 000259 и РФФИ № 01-0100739 . 


\section{Введение}

Теоремы глобальной теории особенностей связывают топологические инварианты многообразий, расслоений и прочих глобальных топологических объектов с геометрией особенностей тех или иных дифференциально-геометрических структур: отображений, сечений расслоений, взаимного расположения подмногообразий и других. Классическим примером служит теорема Хопфа, связьвающая эйлерову характеристику многообразия с особенностями заданного на нем векторного поля. К другим классическим результатам, известным еше с XIX века, относятся, например, теорема Римана-Гурвища, выражающая род римановой поверхности через порядки ветвлений, формулы Плюккера для количества точек перегиба и точек самопересечения плоской кривой и двойственной к ней, а также формулы Сальмона для количества плоскостей, имеющих заданньй тип касания с поверхностью общего положения в трехмерном пространстве.

За последующие полтора века появилось огромное количество новых результатов, которые можно отнести к глобальной теории особенностей, см. обзоры С. Л. Клеймана [29], [32] и книгу У. Фултона [16]. На всем протяжении развития теории топологические и геометрические методы сопутствовали алгебраическим. Топологические идеи давали пищу для интуиции и служили мотивировкой для формулировки новых теорем и гипотез, в то время как алгебраические методы давали их строгое обоснование и перенос на случай произвольного основного поля. С развитием теории пересечений и появлением мошных методов избыточных пересечений, позволяюших иметь дело с произвольными особыми схемами, влияние топологических идей стало заметно ослабевать. В результате в течение длительного времени эта область разрабатывалась исключительно в рамках алгебраической геометрии, совершенно вне связи с топологической подоплекой. Исключение топологических идей из доказательств и даже из мотивировок не пошло на пользу этим исследованиям. Мы надеемся убедить читателей в том, что топологические идеи далеко еще не исчерпаны.

Обычно задачи исчислительной геометрии формулируются следуюшим образом. Имеется пространство параметров (модулей) геометрических объектов, и требуется определить класс когомологий, двойственный циклу на этом пространстве, определяемому теми или иньми условиями вырождений. В большом количестве случаев циклы вырождений можно интерпретировать как циклы особенностей (или мультиособенностей) заданного типа для некоторого гладкого отображения $f: M \rightarrow N$.

Например, пусть задано гладкое проективное многообразие $V \subset \mathbb{C} P^{n}$ и мы изучаем особенности его пересечения со всевозможньми $k$-мерными подпространствами. Тогда в качестве $N$ можно взять грассманиан $G_{k+1, n+1}$ всех $k$-мерных проективных подпространств в $\mathbb{C} P^{n}$, в качестве $M$ - многообразие инцидентности, образованное парами вида (точка многообразия $V$, проходящее через эту точку $k$-мерное подпространство), а в качестве $f$ - естественную проекцию.

Если построенное отображение $f$ удовлетворяет некоторому условию общности положения, то оно имеет лишш стандартные вырождения, локальное строение которых хорошо изучено в теории особенностей. Естественно ожидать, что классы когомологий, двойственные циклам этих вырождений, имеют универсальное выражение в терминах глобальных топологических инвариантов, связанных с многообразиями $M, N$ и отображением $f$, и не зависят от конкретных геометрических задач, к которым по- 
лученные формулы применяются. Сформулированный принцип лежит в основе всех результатов этой статьи. Он дает единьй подход к большому ряду задач исчислительной геометрии, не имеюших, на первый взгляд, ничего общего. Кроме того, его применение существенно облегчает задачу нахождения универсальных формул и, тем самым, решение поставленных исчислительных задач.

Изучая циклы локальных особенностей гладкого отображения $f: M \rightarrow N$ вещественных многообразий, Р. Том предположил, что класс $\mathbb{Z}_{2}$-когомологий, двойственньй циклу $\Sigma \subset M$ произвольной локальной особенности, должен выражаться как универсальный многочлен (называемый теперь многочленом Тома) от классов Штифеля-Уитни $\omega_{i}(M), f^{*}\left(\omega_{j}(N)\right)$ отображаемых многообразий [51], [19]. Вычислению многочленов Тома для различных конкретных типов особенностей было посвящено большое количество работ 70-80-х годов (см. [43], [44], [49], [13], [17], [1], [2], а также обзор [6]). Обычно для нахождения этих многочленов использовалось подходящее разрешение $\widetilde{\Sigma} \rightarrow \Sigma$ цикла особенностей. Класс, двойственньй гладкому многообразию $\widetilde{\Sigma}$ в объемлюшем пространстве разрешения, вычислить бьвает значительно проще. Для нахождения искомого выражения для класса, двойственного $\Sigma$, применяется гомоморфизм Гизина проекции пространства разрешения на $M$.

Приведенньй метод одинаково успешно может быть применим в комплексной ситуации голоморфных отображений и их особенностей. В этом случае класс, двойственный циклу особенностей, является целочисленным и выражается как универсальньй многочлен от классов Чженя $c_{i}(M), f^{*}\left(c_{j}(N)\right)$. Одним из следствий вычислений является тот факт, что для большого класса особенностей многочлен Тома, в действительности, выражается не через сами классы Чженя многообразий, а через их определенные комбинации, а именно через относительные классы Чженя $c_{i}(f)=c_{i}\left(f^{*} T N-T M\right)$, определенные формальным равенством

$$
1+c_{1}(f)+c_{2}(f)+\cdots=\frac{1+f^{*} c_{1}(N)+f^{*} c_{2}(N)+\cdots}{1+c_{1}(M)+c_{2}(M)+\cdots}
$$

(в вешественном случае, соответственно, через относительные классы Штифеля-Уитни $\left.\omega_{i}(f)=\omega_{i}\left(f^{*} T N-T M\right)\right)$.

С ростом коразмерности особенности находить подходящее разрешение становится все труднее, и метод разрешения наталкивается на серьезные технические трудности. Сравнительно недавно Р. Римани [46], [47] предложил другой, косвенньй, метод вычисления многочленов Тома, которьй использует теорему об их сушествовании. Идея метода очень проста. Для определения многочлена достаточно вычислить его коэффициенты. Для вычисления коэффициентов достаточно рассмотреть ряд примеров отображений, в которых как классы Чженя, так и классы, двойственные циклам особенностей, можно вычислить явно. Всякий такой пример дает линейные соотношения на коэффициенты многочлена Тома, и при удачном выборе примеров эти соотношения определяют многочлен полностью. Метод Римани оказался чрезвычайно эффективным и позволил получить многочлены Тома практически для всех расклассифищированных особенностей.

Несомненно, факт существования многочлена Тома является универсальньм и справедлив в абстрактной ситуации алгебраических многообразий для произвольного алгебраически замкнутого основного поля и для колец Чжоу вместо когомологий. 
Тем не менее, доказательство существования многочлена Тома для комплексных отображений, которое использовало бы лишш алгебраические методы теории пересечений, до сих пор не опубликовано.

Одно из возможных топологических доказательств сушествования многочлена Тома приведено в статье [24], см. также п. 6. Это доказательство состоит в построении так назьваемого классифицирующего пространства особенностей, конечномерные аппроксимации которого являются алгебраическими многообразиями, имеющими топологию грассманиана. В качестве такой аппроксимации можно взять пространство $K$-струй ростков $m$-мерных подмногообразий в начале координат аффинного пространства $\mathbb{C}^{n}$, где $n \gg m \gg 0$. Классифицируюшее пространство имеет богатую геометрию. На нем имеется разбиение, страты которого находятся во взаимно однозначном соответствии с классами эквивалентности особенностей отображений. Страт, отвечающий данной особенности $\alpha$, состоит из $K$-струй подмногообразий, проекция которых на фиксированное координатное подпространство $\mathbb{C}^{m+\ell} \subset \mathbb{C}^{n}$ имеет в начале координат особенность $\alpha$. Далее мы определяем многочлен Тома особенности $\alpha$ как класс когомологий, двойственный замыканию соответствующего страта в классифицируюшем пространстве и выраженный через мультипликативные образуюшие когомологий грассманиана. Для всякого голоморфного отображения $f: M \rightarrow N$ имеется классифицирующее отображение $\varkappa$ из $M$ в классифицирующее пространство. Это отображение индуцирует как классы когомологий, двойственные циклам особенностей, так и характеристические классы Чженя. Существование классифищирующего отображения доказьвает универсальность многочленов Тома.

Несмотря на то что существование многочленов Тома известно в течение уже почти полувека, до недавнего времени не было известно аналогичного априорного выражения для классов, двойственных циклам мультиособенностей. Получению такого выражения и посвящена данная статья.

Теория мультиособенностей имеет богатую историю. К числу ранних результатов можно отнести упомянутые вьше теоремы Плюккера и Сальмона. Для случая общих отображений одним из первых результатов является формула для класса цикла двойных точек

$$
m_{2}=f^{*} f_{*} 1-c_{\ell}(f), \quad \ell=\operatorname{dim} N-\operatorname{dim} M .
$$

В разной степени общности (вначале - для гладких отображений при помощи топологических соображений, затем - для морфизмов произвольных особых схем с использованием формул избыточного пересечения) эту формулу получили Тодд, Уитни, Ронга, Лаксов, Хольм, Джонсон, Фултон (см. исторические замечания в [16], [30]).

Следующий результат - формула Герберта-Ронга для циклов кратных точек иммерсий,

$$
m_{r}=f^{*} n_{r-1}-c_{\ell}(f) m_{r-1} .
$$

Эта формула также была вначале получена из топологических соображений и лишш затем передоказана при помоши формул избыточного пересечения.

Для отображений, допускаюших особенности, общие формулы впервые получил Клейман [30], [31]. Как заметил Кащ [21], формулу Клеймана для кратных точек можно записать в виде

$$
m_{r}=f^{*} n_{r-1}+\sum_{i=1}^{r-1} p_{i}(c(f)) m_{r-i}
$$


где $p_{i}$ - универсальные многочлены от относительных классов Чженя $c_{i}(f)$, которые не зависят от $r$ и вычисляются по определенному алгоритму. Для применимости метода, при помощи которого эта формула была получена, необходимо предположить, что отображение $f$ не имеет особенностей коранга, большего 1 (или что этими особенностями можно пренебречь по соображениям размерностей), см. [33]. Сравнительно простая замкнутая формула для этих многочленов получена в [26].

Римани показал [47], что наличие особенностей коранга 2 приводит к нарушению равенства (1) в первом же случае, когда это возможно по соображениям размерностей (при $\operatorname{dim} N-\operatorname{dim} M=1, r=6)$. Однако, как заметил Римани, равенство (1) остается справедливым для общих отображений при $r \leqslant 7$, если несколько подправить многочлены $p_{6}, p_{7}$. Поправочные слагаемые имеют носитель на многообразии $\Sigma^{2}$ особенностей коранга, большего или равного 2, и потому они обрашаются в нуль для всякого отображения коранга 1. Одно из следствий теории мультиособенностей, изложенной в этой статье, заключается в том, что для подходящего выбора многочленов $p_{i}$ формула (1) остается справедливой для произвольных общих отображений, более того, многочлены $p_{i}$ определяются этим условием однозначно.

Результаты Клеймана были перенесены на случай произвольных мультиособенностей отображений коранга 1 в работе Колли [14]. Приведенньй в этой работе алгоритм дает решение задачи нахождения формулы для произвольной мультиособенности, но получаемые в результате формулы имеют весьма громоздкий вид. В более удобном замкнутом виде эти формулы приведены в [26]. Как и формулы Клеймана, эти формулы применимы только для отображений коранга 1, однако даже в этом случае они нашли важные применения в исчислительной геометрии [15].

В наиболее общем виде формула для мультиособенностей, применимая к общим голоморфньм отображениям без ограничений на сложность встречающихся особенностей (при вьполнении условия общности отображения), получена автором. Для формулировки этой формулы уточним понятие цикла мультиособенностей.

Мультиособенности нумеруются наборами локальных особенностей $\underline{\alpha}=\left(\alpha_{1}, \ldots\right.$, $\left.\alpha_{r}\right)$. С каждым типом мультиособенностей $\underline{\alpha}$ можно связать соответствуюший ему цикл в многообразии $M$ области определения отображения. Этот цикл параметризует такие точки $x_{1} \in M$, что существуют дополнительные попарно различные точки $x_{2}, \ldots, x_{r} \in M$, имеющие тот же образ $f\left(x_{i}\right)=f\left(x_{1}\right)$ и такие, что $f$ имеет особенность $\alpha_{i}$ в $x_{i}$ при $i=1, \ldots, r$. Обозначим через $\bar{m}_{\underline{\alpha}} \in H^{*}(M), \bar{n}_{\underline{\alpha}} \in H^{*}(N)$ классы когомологий, двойственные замыканию этого цикла и его (приведенного) образа соответственно. Этот цикл отображается на свой образ с кратностью $k$, равной числу вхождений особенности $\alpha_{1}$ в набор $\underline{\alpha}$. Отсюда вытекает очевидное соотношение

$$
\bar{n}_{\underline{\alpha}}=k f_{*} \bar{m}_{\underline{\alpha}}
$$

где $f_{*}$ - гомоморфизм прямого образа, или гомоморфизм Гизина, который определен, если отображение $f$ собственно. Если все классы $\alpha_{i}$ набора являются классами иммерсии (обозначаемыми нами через $A_{0}$ ), то соответствующие классы

$$
\left.\bar{m}_{r}=\bar{m}_{(\underbrace{A_{0}, \ldots, A_{0}}_{r})}, \quad \bar{n}_{r}=\bar{n}_{(\underbrace{A_{0}, \ldots, A_{0}}_{r}}\right)
$$


называются классами $r$-кратных точек в прообразе $M$ и образе $N$ соответственно.

Классы $\bar{m}_{\underline{\alpha}}, \bar{n}_{\underline{\alpha}}$ часто бьвает удобно рассматривать с их естественными кратностями, и мы полагаем

$$
m_{\underline{\alpha}}=\# \operatorname{Aut}\left(\alpha_{2}, \ldots, \alpha_{r}\right) \bar{m}_{\underline{\alpha}}, \quad n_{\underline{\alpha}}=f_{*} m_{\underline{\alpha}}=\# \operatorname{Aut}\left(\alpha_{1}, \ldots, \alpha_{r}\right) \bar{n}_{\underline{\alpha}},
$$

где \# Aut - число элементов в группе симметрий соответствуюшего мультииндекса.

Основной результат теории мультиособенностей (теорема 2.2) заключается в том, что для всякого типа мультиособенностей $\underline{\alpha}$ класс $m_{\underline{\alpha}}$ задается универсальным полиномиальным выражением, в котором участвуют относительные классы Чженя $c_{i}(f)=$ $c_{i}\left(f^{*} T N-T M\right)$, а также классы Ландвебера-Новикова, получаемые из всевозможных мономов от относительных классов Чженя применением гомоморфизма $f^{*} f_{*}$.

Более того, в уточненной формулировке теоремы 3.2 утверждается, что это полиномиальное выражение для класса $m_{\underline{\alpha}}$ имеет довольно специальньй вид. Для всякого набора особенностей $\underline{\alpha}=\left(\alpha_{1}, \ldots, \alpha_{r}\right)$ сушествует универсальный многочлен $R_{\underline{\alpha}}$ от классов $c_{i}=c_{i}(f)$, назьваемьй нами остаточнылм многочленом мультиособенности $\underline{\alpha}$, такой, что класс мультиособенности $m_{\underline{\alpha}}$ выражается следуюшим образом через остаточные многочлены всевозможных поднаборов данного набора $\underline{\alpha}$ :

$$
m_{\underline{\alpha}}=\sum_{J_{1} \sqcup \cdots \sqcup J_{k}=\{1, \ldots, r\}} R_{\underline{\alpha}_{J_{1}}} f^{*} f_{*} R_{\underline{\alpha}_{J_{2}}} \cdots f^{*} f_{*} R_{\underline{\alpha}_{J_{k}}},
$$

где суммирование производится по всевозможным разбиениям множества $\{1, \ldots, r\}$ в несвязное объединение непустых неупорядоченных подмножеств $\{1, \ldots, r\}=J_{1} \sqcup$ $\cdots \sqcup J_{k}, k \geqslant 1$, и где подмножество, содержащее элемент $1 \in\{1, \ldots, r\}$, обозначается через $J_{1}$.

Для вычисления остаточных многочленов можно применить метод Римани использования симметрий. Дополнительные соображения, позволяюшие приспособить этот метод для исследования мультиособенностей, обсуждаются в п. 5. Полные таблищы всех вычисленных остаточных многочленов занимают большое количество страниц многострочных формул, их можно найти в [28]. Для мультиособенностей невысоких коразмерностей (и для $\ell=0,1$ ) эти многочлены приведены в таблицах п. 5.

Приведенное в п. 6 топологическое доказательство теоремы 2.2 основано на глубокой связи теории мультиособенностей отображений с теорией комплексных кобордизмов и когомологических операций. Эта связь использовалась, по существу, еше в классической конструкции Понтрягина, сводящей вычисление гомотопических групп сфер к классификашии оснащенных кобордизмов. В.И. Арнольд сообшил мне, что он обсуждал около 1967 года с В.А. Рохлиным возможность обобшения конструкции Понтрягина на случай произвольных отображений с особенностями. Позже он использовал эту связь при вычислении групп лагранжевых и лежандровых кобордизмов [3] и гомологий пространств функций без сложных особенностей [4]. Идеи Арнольда развивались затем В. А. Васильевым [54] и автором. В школе Рохлина аналогичные идеи независимо развивал А. Сюч. В наиболее формализованном виде теория Сюча изложена в его совместной с Р. Римани работе [48].

Главное наблюдение, на котором основаны результаты настоящей статьи, заключается в том, что искомое классифицируюшее пространство для мультиособенностей, построению которого, в частности, посвяшена статья [48], совпадает по существу 
с давно построенным Р. Томом классифицирующим пространством кобордизмов [51] (конструкция которого также обобщает конструкцию Понтрягина). Для случая голоморфных отображений и комплексных кобордизмов это пространство является итерированным пространством петель $\Omega^{2 m} M U(m+\ell), m \rightarrow \infty$, где $M U(m)$ - пространство Тома классифицирующего векторного расслоения ранга $m$ над комплексньм грассманианом $B U(m)=G_{m, \infty}$. Это наблюдение значительно упрощает исследование топологии этого пространства. Классифицирующее пространство мультиособенностей разбито на страты, отвечающие различным мультиособенностям, аналогично тому, как классифицируюшее пространство локальных особенностей разбито на страты, отвечаюшие различным классам локальных особенностей. Отсюда следует, что характеристический класс всякой мультиособенности задается классом когомологий классифицирующего пространства. Таким образом, для получения универсальной формулы для классов мультиособенностей необходимо описать кольцо когомологий пространства $\Omega^{2 m} M U(m+\ell)$.

Задача когомологического исследования груп кобордизмов является не новой. В статье В.М. Бухштабера [10] приведено решение этой задачи при помощи явной формулы для характера Чженя-Дольда. Согласно этой формуле, класс кобордизма голоморфного отображения $f: M \rightarrow N$ определяется однозначно с точностью до кручения набором когомологических классов на $N$, задаваемых операциями Ландвебера-Новикова со значениями в когомологиях. Таким образом, из описанной вьше связи мультиособенностей и кобордизмов вытекает, что значения когомологических классов Ландвебера-Новикова однозначно определяют класс когомологий всякой мультиособенности. К аналогичньм вьводам приводят и прямые вычисления кольца когомологий пространства $\Omega^{2 m} M U(m+\ell)$, проведенные в п. 6. Мы показываем, что, по крайней мере при $\ell>0$ и в случае рациональных коэффициентов, это кольцо свободно мультипликативно порождено классами Ландвебера-Новикова. Эти вычисления и приводят к формулировке теоремы 2.2 .

В п. 7 обсуждаются также различные подходы к доказательству теорем 2.2 и 3.2 при помощи методов теории пересечений. Мы уточняем определение схемы мультиособенностей, приводим точную формулировку условия обшности отображения и даем некоторые гипотетические обобщения теоремы 3.2. К настоящему времени довести эти соображения до строгости и получить доказательства всех приведенных утверждений методами теории пересечений, а также привести явную формулу для остаточных многочленов удалось только для частного случая отображений, коранг которых (т.е. размерность ядра производной) не превосходит 1 ни в одной точке, [26].

В п. 8 формулируется универсальная формула для мультиособенностей гиперповерхностей и для мультиособенностей лежандровых отображений. Эта формула аналогична (2) за исключением того, что остаточные многочлены $R_{\underline{\alpha}}$ в этом случае - универсальные лежандровы характеристические классы. Непосредственные применения этой формулы дают сотни новых результатов об исчислении особенностей дивизоров в обших линейных системах на неособых проективных многообразиях. В задаче исчисления особенностей кривых на поверхностях наши вычисления согласуются с известными результатами П. Ле Барца [38], С. Дж. Колли [14], [15], И. Вайнзенкера [53], Л. Гёттше [18], Л. Капорасо и Дж. Харриса [12], С. Л. Клеймана и Р. Пино [34], [35], 
а также многих других авторов, внесших вклад в решение этих задач. В частности, наша формула дает обоснование гипотезы Гёттше о числе плоских нодальных кривых, а также различных ее обобщений и вариаций, принадлежащих Клейману и Пино.

Другое приложение полученной формулы - исчисление особенностей касания гиперповерхностей в $\mathbb{C} P^{n}$ с проективньми подпространствами. Эти результаты обобщают на случай $n>3$ классические формулы Плюккера $(n=2)$ и Сальмона $(n=3)$. Простейший вопрос, ответ на которьй до сих пор, по-видимому, не был известен, это вопрос о числе гиперплоскостей в $\mathbb{C} P^{4}$, касающихся в 4 точках заданной общей гиперповерхности степени $d$. Согласно нашим выгислениям, при $d=3$ это число равно 495. Частично полученные результаты представлены в таблищах п. 10. Полный список имеюшихся соотношений занимает десятки страниц и имеется в [28].

Мы признаем, что приведенные в этой статье топологические доказательства общих теорем о характеристических классах особенностей довольно схематичны и неполны. Вся необходимая техника для преодоления возникающих трудностей имеется, однако продвижение в этом направлении не кажется столь уж обязательным: в окончательном варианте теория мультиособенностей должна использовать все же язык алгебраической геометрии, а излагаемые топологические аргументы следует рассматривать, скорее, как мотивировку. Насколько мне известно, даже для случая локальных особенностей доказательство существования многочленов Тома не переложено на язык алгебраической геометрии. Тем не менее, многочисленные топологические и численные подтверждения не оставляют сомнения, что приведенные результаты справедливы также в абстрактной алгебраической постановке для произвольного алгебраически замкнутого основного поля и для колец Чжоу вместо когомологий. Сальмон получил свои формулы еще в середине XIX века, однако доказательство, которое удовлетворило бы современных алгебраистов, появилось сравнительно недавно [50]. Хочется надеяться, что временной разрьв между формулируемыми здесь результатами и их обоснованием на язьке теории пересечений будет менњше, чем для упомянутых вьше исчислительных результатов Сальмона.

Создание теории, представленной в работе, было бы невозможно без сотрудничества с математиками самых различных специальностей - теории особенностей, алгебраической геометрии, топологии. Я особенно благодарен В.И. Арнольду, В.М. Бухштаберу, В.А. Васильеву, Вал. С. Куликову, С.Л. Клейману, Д. Монду, P. Пино, Д. ван Стратену, общение с которьми чрезвыгайно помогло мне в работе.

\section{1. Многочлены Тома локальных особенностей}

Мы рассматриваем особенности голоморфного отображения $f: M \rightarrow N$ гладких комплексных многообразий фиксированной относительной размерности

$$
\ell=\operatorname{dim} N-\operatorname{dim} M .
$$

Одним из самых важных инвариантов ростка $f:\left(\mathbb{C}^{m}, 0\right) \rightarrow\left(\mathbb{C}^{n}, 0\right)$ является его локальная алгебра

$$
Q_{f}=\mathscr{O}_{\mathbb{C}^{m}, 0} / f^{*} \mathfrak{m}_{\mathbb{C}^{n}, 0}
$$

факторалгебра кольца $\mathscr{O}_{\mathbb{C}^{m}, 0}$ ростков функций в начале координат многообразия области определения по идеалу, порожденному компонентами $f_{i}, i=1, \ldots, n$, отображения $f$. 
Напомним [6], что два ростка голоморфных отображений $f_{i}:\left(\mathbb{C}^{m_{i}}, 0\right) \rightarrow\left(\mathbb{C}^{m_{i}+\ell}, 0\right)$ называются стабильно $\mathscr{K}$-әквивалентными, если их локальные алгебры изоморфны. Классы стабильной $\mathscr{K}$-эквивалентности задают разбиение пространства $K$-струй отображений. Особенности этого разбиения не зависят, с точностью до диффеоморфизма и умножения на аффинное пространство подходящей размерности, ни от представителя класса стабильной $\mathscr{K}$-эквивалентности, ни от $K$, если $K$ достаточно велико, но существенно зависят от $\ell$. Таким образом, для различных $\ell$ имеются независимые $\mathscr{K}$-классификации ростков отображений.

Корангом ростка $f:\left(\mathbb{C}^{m}, 0\right) \rightarrow\left(\mathbb{C}^{m+\ell}, 0\right)$ называется размерность ядра производной в начале координат. Коранг равен также минимальному числу образуюших локальной алгебры, или минимальному значению $c$, для которого данный класс $\mathscr{K}$-эквивалентности может быть реализован ростком отображения $\left(\mathbb{C}^{c}, 0\right) \rightarrow\left(\mathbb{C}^{c+\ell}, 0\right)$.

Для особенностей малой коразмерности классификация дискретна, но в большой коразмерности (например, при $\ell=0$ - начиная с коразмерности 9) имеются непрерывные семейства неэквивалентных алгебр, поэтому понятие класса особенности следует уточнить.

1.1. ОПРеДЕлЕнИЕ. Стабильным классом К -особенностей называется семейство $\alpha$ ростков отображений $\left(\mathbb{C}^{m}, 0\right) \rightarrow\left(\mathbb{C}^{m+\ell}, 0\right)$, заданных при всех $m=1,2, \ldots$, которое инвариантно относительно стабильной $\mathscr{K}$-эквивалентности (т.е. два стабильно эквивалентных ростка принадлежат или не принадлежат $\alpha$ одновременно) и которое, кроме того, удовлетворяет следуюшим условиям.

(i) Коранг $c$ всех ростков данного класса одинаков. Таким образом, для описания класса $\alpha$ достаточно задать его представителей $\left(\mathbb{C}^{m}, 0\right) \rightarrow\left(\mathbb{C}^{m+\ell}\right)$ для $m=c$.

(ii) Класс $\alpha$ является конечно определенньм, т.е. вопрос о принадлежности данного ростка классу $\alpha$ должен решаться по его $K$-струе, где $K$ зависит от класса $\alpha$ и не зависит от его конкретного представителя. Мы вовсе не требуем, чтобы каждое отображение класса $\alpha$ было конечноопределенньм, однако можно показать, что для достаточно больших $K$ множество ростков, для которых $K$-струя достаточна, образует плотное подпространство в классе $\alpha$.

(iii) $K$-струи ростков отображений класса $\alpha$ должны образовьвать полуалгебраическое гладкое (возможно, незамкнутое) подмногообразие в пространстве $K$-струй ростков отображений $\left(\mathbb{C}^{m}, 0\right) \rightarrow\left(\mathbb{C}^{m+\ell}\right)$, если $K$ достаточно велико. (В этом случае коразмерность $\operatorname{codim} \alpha$ этого многообразия не зависит от $K, m$ и называется коразмерностью особенности $\alpha$.)

Примерами классов, состояших больше чем из одной орбиты $\mathscr{K}$-эквивалентности, служат классы Тома-Бордмана, см. [6].

Пусть $f: M \rightarrow N$ - голоморфное отображение неособых комплексных многообразий. Точки многообразия $M$ области определения классифицируются в соответствии с локальными особенностям отображения $f$. Для заданного класса $\mathscr{K}$-особенностей $\alpha$ обозначим через $M(\alpha) \subset M$ замыкание множества точек, в которых $f$ имеет особенность $\alpha$. Хорошо известен следующий обший принцип, автором которого является Р. Том.

1.2. Теорема [52], [19]. Для всякого общего отображсения класс когомологий, двойственный по Пуанкаре циклу $M(\alpha)$, может быть выражен как 
универсальный многочлен (так называемый многочлен Тома) от переменных $c_{1}, c_{2}, \ldots$, вычисленный на относительных классах Чжсеня отображения $c_{i}(f)=c_{i}\left(f^{*} T N-T M\right)$.

Одно из возможных доказательств этой теоремы приведено в п. 6. Условие общности положения состоит в том, что струйное расширение отображения $f$ трансверсально замыканию цикла особенностей $\alpha$ в соответствующем расслоении струй. В другом варианте, можно предположить, что особое подмногообразие $M(\alpha) \subset M$ имеет ожидаемую коразмерность $\operatorname{codim} \alpha$. В этом случае неприводимые компоненты $M(\alpha)$ необходимо брать с кратностями, предписанными структурой схемы на этих компонентах. В обшем случае мы можем гарантировать только, что характеристический класс, заданньй многочленом Тома, может быть представлен замкнутым циклом с носителем на $M(\alpha)$. Подобное замечание справедливо и по отношению к другим циклам вырождений, рассматриваемым в этой статье.

1.3. ПримеР. Обозначим через $\Sigma^{r} \subset M$ цикл точек, в которых размерность ядра производной $f_{*}$ отображения не менее $r$. Классы, двойственные этим циклам, называются классами Портеуса-Тома. Исторически они были одними из первых вычисленных многочленов Тома. Выражение для этих классов имеет вид определителя:

$$
\left[\Sigma^{r}\right]=\operatorname{det}\left|c_{r+\ell+j-i}(f)\right|_{i, j=1, \ldots, r} .
$$

Стандартное доказательство этого равенства использует разрешение особенностей цикла $\Sigma^{r}$. А именно, рассмотрим многообразие $\widetilde{\Sigma}^{r}$, образованное парами вида (точка $x \in M, r$-мерная плоскость в $\left.\operatorname{ker} f_{*}(x) \subset T_{x} M\right)$. Естественная проекция $p: \widetilde{\Sigma}^{r} \rightarrow \Sigma^{r}$ является взаимно однозначной над открытой частью цикла $\Sigma^{r}$, в которой ядро производной в точности $r$-мерно. Следовательно, класс Портеуса-Тома вычисляется при помоши гомоморфизма Гизина

$$
\left[\Sigma^{r}\right]=p_{*}\left[\widetilde{\Sigma}^{r}\right]
$$

Гомоморфизм Гизина $p_{*}: H^{*}\left(\widetilde{\Sigma}^{r}\right) \rightarrow H^{*}(M)$ действует в “неестественную” сторону и определяется как композиция изоморфизма двойственности Пуанкаре на $\widetilde{\Sigma}^{r}$, обычного гомоморфизма $p_{*}$ в гомологиях и изоморфизма двойственности Пуанкаре на $M$. Этот гомоморфизм сдвигает градуировку на разницу (вещественных) размерностей многообразий; корректность его определения вытекает из собственности отображения $p$ (многообразия $M, \widetilde{\Sigma}^{r}$ при этом не обязаны быть компактньми). Для вычисления гомоморфизма Гизина разложим проекцию $p$ в композицию $p=\pi \circ i$, где $i$ - вложение многообразия $\widetilde{\Sigma}^{r}$ в пространство грассманова расслоения $G_{r}(T M)$, ассоциированного с векторным расслоением $T M$, и $\pi: G_{r}(T M) \rightarrow M$-естественная проекция на базу расслоения. Обозначим через $\tau$ тавтологическое подрасслоение ранга $r$ в $\pi^{*} T M$ и через $Q$ соответствующее факторрасслоение ранга $m-r$. Производная отображения $f$ задает естественньй морфизм расслоений $\tau \rightarrow f^{*} T N$. По определению, многообразие $\widetilde{\Sigma}^{r}$ определяется как множество нулей этого морфизма, т.е. как множество нулей сечения расслоения $\operatorname{Hom}\left(\tau, f^{*} T N\right)$. При вьполнении условия обшности положения это сечение трансверсально нулевому. В частности, подмногообразие $\widetilde{\Sigma}^{r} \subset G_{r}(T M)$ гладко и его двойственньй класс когомологий задается как старший класс Чженя этого расслоения,

$$
i_{*}\left[\widetilde{\Sigma}^{r}\right]=c_{r n}\left(\operatorname{Hom}\left(\tau, f^{*} T N\right)\right)=\operatorname{det}\left|c_{n-j+k}\left(f^{*} T N-\tau\right)\right|_{j, k=1, \ldots, r} .
$$


Последнее равенство легко вытекает из принципа расщепления и может рассматриваться как упражнение из теории характеристических классов. Далее, представив $c_{k}\left(f^{*} T N-\tau\right)=c_{k}\left(f^{*} T N-T M+Q\right)=\sum_{j} c_{j}(Q) c_{k-j}(f)$ и подставив полученные выражения в определитель, мы получаем

$$
i_{*}\left[\widetilde{\Sigma}^{r}\right]=c_{m-r}^{r}(Q) \operatorname{det}\left|c_{n-j+k-m+r}(f)\right|_{j, k=1, \ldots, r}+\cdots,
$$

где многоточие обозначает члены строго меньших степеней относительно классов $c_{k}(Q)$. Гомоморфизм $\pi_{*}$ обращается в нуль на этих классах по соображениям размерностей и переводит $c_{m-r}^{r}(Q)$ в $1 \in H^{0}(M)$ (последнее утверждение является проявлением того факта, что через $r$ общих точек в векторном пространстве проходит единственное $r$-мерное векторное подпространство). Следовательно, мы получаем, окончательно,

$$
\left[\Sigma^{r}\right]=\pi_{*} i_{*}\left[\widetilde{\Sigma}^{r}\right]=\operatorname{det}\left|c_{n-j+k-m+r}(f)\right|_{j, k=1, \ldots, r} .
$$

Особенности Портеуса-Тома определяются по 1-струе отображения. Описанный вьше метод разрешения особенностей может применяться и для нахождения многочленов Тома классов особенностей, определяемых в терминах высших струй. Именно этим методом были впервые вычислены многочлены Тома для многих особенностей, см. обзор [6]. Тем не менее, с ростом коразмерности особенности находить подходящее разрешение становится все труднее и этот метод наталкивается на технические трудности. Сравнительно недавно Римани предложил альтернативньй косвенньй метод для нахождения многочленов Тома, который использует теорему об их существовании. Метод Римани оказался более эффективным и позволил вычислить многочлены Тома практически всех расклассифищированных особенностей. Вариант этого метода, приспособленный для изучения мультиособенностей, изложен в п. 5 .

В этой статье мы обобщаем теорему 1.2 и приводим подобное априорное описание классов когомологий, двойственных циклам мультиособенностей.

\section{2. Формулировка основной теоремы для мультиособенностей}

Мультиособенностью назьвается произвольньй конечный упорядоченньй набор локальных особенностей. С каждым классом мультиособенностей $\underline{\alpha}=\left(\alpha_{1}, \ldots, \alpha_{r}\right)$ можно связать соответствуюший иикл мультиособенностей $M(\underline{\alpha})$ заданного отображения $f: M \rightarrow N$ относительной размерности $\ell=\operatorname{dim} N-\operatorname{dim} M$. Он определяется как (особое) подмногообразие в декартовом произведении $M^{r}=M \times \cdots \times M$, задаваемое как замькание множества наборов попарно различных точек $\left(x_{1}, \ldots, x_{r}\right) \in$ $M^{r}, x_{i} \neq x_{j}$ при $i \neq j$, таких, что $f\left(x_{1}\right)=\cdots=f\left(x_{r}\right)$ и $f$ имеет особенность заданного типа $\alpha_{i}$ в $x_{i}$ при $i=1, \ldots, r$. Приведенное определение применимо, вообще говоря, только для отображений, удовлетворяющих условию обшности (это условие, а также более общее определение многообразия $M(\underline{\alpha})$ обсуждаются в п. 7). При вьполнении этого условия $M(\underline{\alpha})$ имеет ожидаемую размерность, равную $\operatorname{dim} M-\sum \operatorname{codim} \alpha_{i}-$ $(r-1) \ell$, где $\operatorname{codim} \alpha_{i}$ - ожидаемая коразмерность цикла локальной особенности $\alpha_{i}$ в многообразии $M$ области определения отображения, равная истинной коразмерности многообразия этой особенности в пространстве струй отображений.

Рассмотрим естественные проекции $p_{M}: M(\underline{\alpha}) \rightarrow M$ и $p_{N}: M(\underline{\alpha}) \rightarrow N$, задаваемые проекцией на первьй сомножитель в декартовом произведении $M^{r}$ и отображением 
$f$ соответственно. Если $f$ собственно, то образы этих проекций - замкнутые особые подмногообразия в $M$ и $N$. Теория характеристических классов мультиособенностей описьвает классы когомологий $\bar{m}_{\underline{\alpha}} \in H^{*}(M), \bar{n}_{\underline{\alpha}} \in H^{*}(N)$, двойственные образам $p_{M}(M(\underline{\alpha})) \subset M, p_{N}(M(\underline{\alpha})) \subset N$ соответственно (рассматриваемым как особые приведенные подмногообразия).

Мы часто рассматриваем классы $\bar{m}_{\underline{\alpha}}, \bar{n}_{\underline{\alpha}}$ с их естественньми кратностями, а именно положим

$$
m_{\underline{\alpha}}=\# \operatorname{Aut}\left(\alpha_{2}, \ldots, \alpha_{r}\right) \bar{m}_{\underline{\alpha}}, \quad n_{\underline{\alpha}}=\# \operatorname{Aut}\left(\alpha_{1}, \ldots, \alpha_{r}\right) \bar{n}_{\underline{\alpha}},
$$

где \# $\operatorname{Aut}\left(\alpha_{1}, \ldots, \alpha_{r}\right)=\# \operatorname{Aut}(\underline{\alpha})$ - количество перестановок $\sigma \in S(r)$ таких, что $\alpha_{\sigma(i)}=\alpha_{i}$ при $i=1, \ldots, r$. Если $\underline{\alpha}$ содержит $k_{j}$ особенностей одинакового типа $\beta_{j}$, $j=1,2, \ldots$, с попарно различными $\beta_{j}$, то \# Aut $(\underline{\alpha})=k_{1} ! k_{2} ! \ldots$. Эти кратности равны также степеням отображения цикла мультиособенностей $M(\underline{\alpha})$ на свой образ под действием проекций $p_{M}$ и $p_{N}$ соответственно.

Отметим, что отображение $f$ индуцирует отображение $p_{M}(M(\underline{\alpha})) \rightarrow p_{N}(M(\underline{\alpha}))$. Степень этого отображения равна числу особенностей $\alpha_{1}$ в наборе $\underline{\alpha}=\left(\alpha_{1}, \ldots, \alpha_{r}\right)$. Следовательно, имеет место очевидное соотношение

$$
n_{\underline{\alpha}}=f_{*} m_{\underline{\alpha}} \in H^{*}(N),
$$

где $f_{*}$ - гомоморфизм Гизина (которьй корректно определен, если отображение $f$ собственно).

Приведенная ниже теорема 2.2 выражает классы $\bar{m}_{\underline{\alpha}}, \bar{n}_{\underline{\alpha}}$ через некоторые характеристические классы, связанные с отображением $f$. Эти классы включают в себя, в частности, классы Чженя самих многообразий $c_{i}(T M) \in H^{*}(M)$ и $c_{i}(T N) \in$ $H^{*}(N)$. Больше классов можно получить из этих применением гомоморфизмов обратного и прямого образа $f^{*}: H^{*}(N) \rightarrow H^{*}(M)$ и $f_{*}: H^{*}(N) \rightarrow H^{*}(M)$. В действительности, окончательные выражения включают только определенные комбинации этих классов, задаваемые следующим образом.

На многообразии $M$ области определения заданы относительные классы Чжсеня $c_{i}(f)=c_{i}\left(f^{*} T N-T M\right)$ отображения $f$. Со всяким мономом $c^{I}=c_{1}^{i_{1}} c_{2}^{i_{2}} \cdots$ от образующих $c_{1}, c_{2}, \ldots$ мы связываем независимую образующую $s_{I}$. Каждая такая переменная определяет на многообразии $N$ области значений следующий класс когомологий (называемый классом Ландвебера-Новикова):

$$
s_{I}(f)=f_{*}\left(c_{1}(f)^{i_{1}} c_{2}(f)^{i_{2}} \ldots\right) \in H^{2 \operatorname{deg} s_{I}}(N),
$$

$\operatorname{deg} s_{I}=\ell+\operatorname{deg} c^{I}=\ell+i_{1}+2 i_{2}+\cdots$, где $\ell=\operatorname{dim} N-\operatorname{dim} M$.

2.1. ЗАмЕчАНИЕ. В действительности, в исходном определении классы Ландвебера-Новикова принимают значения в комплексных кобордизмах [37], [42]; здесь же мы рассматриваем только их аугменташию в обычных когомологиях.

Мы рассматриваем $H^{*}(M)$ как $H^{*}(N)$-модуль посредством действия, задаваемого гомоморфизмом $f^{*}$. Имея это в виду, мы часто используем одно и то же обозначение для класса $\lambda$ из $H^{*}(N)$ и для его обратного образа $f^{*} \lambda$ из $H^{*}(M)$. В частности, мы сохраняем обозначение $s_{I}(f)$ для соответствуюших классов Ландвебера-Новикова 
$f^{*} s_{I}(f)=f^{*} f_{*}\left(c^{I}(f)\right) \in H^{*}(M)$ на многообразии области определения отображения.

Более формально, рассмотрим кольцо $\mathscr{C}=H^{*}(B U)=\mathbb{Z}\left[c_{1}, c_{2}, \ldots\right]$ многочленов от абстрактных классов Чженя и определим новое кольцо

$$
\mathscr{S} \mathscr{C}=\operatorname{Sym} \mathscr{C}=1 \oplus \mathscr{C} \oplus \operatorname{Sym}^{2} \mathscr{C} \oplus \cdots
$$

многочленов от абстрактных классов Ландвебера-Новикова. Естественное линейное отображение $\mathscr{C} \rightarrow \mathscr{S} \mathscr{C}$, переводящее многочлен от классов Чженя в соответствующую линейную комбинацию классов Ландвебера-Новикова, продолжается до гомоморфизма $\mathscr{S} \mathscr{C}$-модулей

$$
s: \mathscr{C} \otimes \mathscr{S} \mathscr{C} \rightarrow \mathscr{S} \mathscr{C}
$$

Кольца $\mathscr{C}, \mathscr{S} \mathscr{C}, \mathscr{C} \otimes \mathscr{S} \mathscr{C}$ градуированы соглашением $\operatorname{deg} c_{i}=i$ и условием того, что $s$ повьшает градуировку на $\ell$.

Для всякого собственного отображения $f: M \rightarrow N$ мы определяем характеристические гомоморфизмы

$$
\mathscr{C} \rightarrow H^{*}(M), \quad \mathscr{S} \mathscr{C} \rightarrow H^{*}(N), \quad \mathscr{C} \otimes \mathscr{S} \mathscr{C} \rightarrow H^{*}(M)
$$

вычисляюшие соответствуюшие характеристические классы, ассоциированные с данным отображением $f$. Эти характеристические гомоморфизмы образуют коммутативную диаграмму

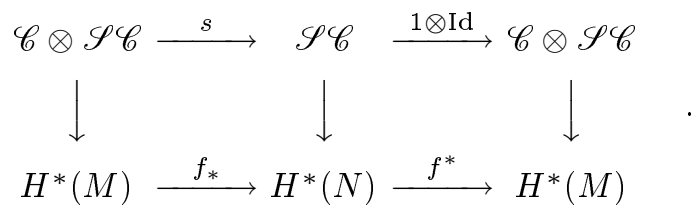

2.2. ТЕОРемА. Для каждого $\ell>0$ и всякого набора $\underline{\alpha}=\left(\alpha_{1}, \ldots, \alpha_{r}\right)$ локальных классов особенностей существует универсальный многочлен $\bar{m}_{\alpha} \in \mathscr{C} \otimes \mathscr{S} \mathscr{C}$ с рациональными коэффициентами от классов Чженя и Ландвебера-Новикова, $c_{i}, s_{I}$, такой, что для всякого достаточно общего собственного голоморфного отображсения $f: M \rightarrow N$ относительной размерности $\ell=\operatorname{dim} N-\operatorname{dim} M$ класс когомологий, двойственный соответствующему циклу мультиособенностей $p_{M}(M(\underline{\alpha})) \subset M$, равен характеристическому классу $\bar{m}_{\underline{\alpha}}$, вычисленному на заданном отображении $f$.

2.3. СлЕДСТВИЕ. В условиях теоремы класс когомологий $\bar{n}_{\underline{\alpha}}$, двойственный ииклу мультиособенностей $p_{N}(M(\underline{\alpha})) \subset N$ в многообразии области значений отображения, задается как универсальный многочлен с рачиональньми коэффичиентами от классов Ландвебера-Новикова отображсения.

Различные уточненные формулировки, учитывающие, в частности, целочисленные классы, сформулированы в виде гипотез в п 7. В частности, соответствующие многочлены для классов $m_{\underline{\alpha}}=\# \operatorname{Aut}\left(\underline{\alpha}^{\prime}\right) \bar{m}_{\underline{\alpha}}$, где $\underline{\alpha}^{\prime}=\left(\alpha_{2}, \ldots, \alpha_{r}\right)$, и $n_{\underline{\alpha}}=\# \operatorname{Aut}(\underline{\alpha}) \bar{n}_{\underline{\alpha}}=$ $f_{*} m_{\underline{\alpha}}$ имеют, по-видимому, целые коэффициенты, и соответствуюшее равенство выполняется, по-видимому, в целочисленных когомологиях. 
Имеется также аналог этой теоремы для отображений неположительной относительной размерности $\ell=\operatorname{dim} N-\operatorname{dim} M \leqslant 0$ [27]. Случай $\ell=0$ обсуждается в п. 5.

Набросок доказательства теоремы, основанного на топологических методах теории кобордизмов, приведен в п. 6. Было бы интересно получить доказательство методами алгебраической геометрии и теории пересечений. В настоящее время такое доказательство получено только для случая отображений, у которых отсутствуют особенности коранга, большего 1 (или у которых такими особенностями можно пренебречь по соображениям коразмерности), см. [26].

\section{3. Уточненная формулировка и остаточные многочлены для мультиособенностей}

Из существования многочленов $m_{\underline{\alpha}}$ и $n_{\underline{\alpha}}$ вытекает, что они должны иметь довольно специальный вид. Рассмотрим $m_{\underline{\alpha}}$ как многочлен от классов Ландвебера-Новикова $s_{I}$, коэффищиенты которого лежат в кольце многочленов от классов Чженя $c_{i}$.

3.1. ОПРЕДЕЛЕНИЕ. Остаточныл многочленом $R_{\underline{\alpha}}$ мультиособенности $\underline{\alpha}=$ $\left(\alpha_{1}, \ldots, \alpha_{r}\right)$ называется многочлен от классов $c_{i}$, равный свободному члену в многочлене $m_{\underline{\alpha}}$ по отношению к переменньм $s_{I}$. Иными словами, $R_{\underline{\alpha}}$ получается из $m_{\underline{\alpha}}$, если в последнем приравнять нулю все образующие $s_{I}$.

Степень $R_{\underline{\alpha}}$ равна коразмерности соответствуюшего цикла мультиособенности в $M$, т.е. $\operatorname{deg} R_{\underline{\alpha}}=\sum \operatorname{codim} \alpha_{i}+(r-1) \ell$ (относительно градуировки, в которой $\operatorname{deg} c_{i}=i$; коразмерность $\operatorname{codim} \alpha_{i}$ вычисляется в пространстве струй отображений $\left.\left(\mathbb{C}^{m}, 0\right) \rightarrow\left(\mathbb{C}^{m+\ell}\right)\right)$.

Обозначим через $S_{\underline{\alpha}}=s\left(R_{\underline{\alpha}}\right) \in \mathscr{S} \mathscr{C}$ соответствуюшую многочлену $R_{\underline{\alpha}}$ линейную комбинацию классов Ландвебера-Новикова. Если $f: M \rightarrow N$-голоморфное собственное отображение, то, как объяснено вьше, многочлены $R_{\alpha}$ и $S_{\underline{\alpha}}$ определяют соответствующие характеристические классы когомологий на $M$ и $N$ соответственно.

3.2. ТЕОРемА. Многочлены $m_{\underline{\alpha}}, n_{\underline{\alpha}}$ однозначно определяются остаточными многочленами $R_{\underline{\alpha}_{J}}=R_{\alpha_{j_{1}}, \ldots, \alpha_{j_{k}}}$ для всевозможниьх подмножеств $J=$ $\left\{j_{1}, \ldots, j_{k}\right\} \subset\{1, \ldots, r\}$. Более явно, имеют место следующие соотношения:

$$
\begin{aligned}
& m_{\underline{\alpha}}=\sum_{J_{1} \sqcup \cdots \sqcup J_{k}=\{1, \ldots, r\}} R_{\underline{\alpha}_{J_{1}}} S_{\underline{\alpha}_{J_{2}}} \cdots S_{\underline{\alpha}_{J_{k}}}, \\
& n_{\underline{\alpha}}=s\left(m_{\underline{\alpha}}\right)=\sum_{J_{1} \sqcup \ldots \sqcup J_{k}=\{1, \ldots, r\}} S_{\underline{\alpha}_{J_{1}}} \cdots S_{\underline{\alpha}_{J_{k}}} .
\end{aligned}
$$

Суммирование производится по всевозможным разбиениям множества $\{1, \ldots, r\}$ в несвязное облединение непустых неупорядоченных подмнохсеств $\{1, \ldots, r\}=J_{1} \sqcup \cdots \sqcup J_{k}, \quad k \geqslant 1$. Подмножество, содерэащее элемент $1 \in\{1, \ldots, r\}$, обозначается через $J_{1}$.

Кроме того, остаточный многочлен $R_{\alpha}$ не зависит от порядка локальных особенностей $\alpha_{i}$, входящих в набор $\underline{\alpha}=\left(\alpha_{1}, \ldots, \alpha_{r}\right)$.

Например, если набор $\underline{\alpha}=\{\alpha\}$ содержит единственный класс особенностей $(r=1)$, то $R_{\alpha}=m_{\alpha}-$ соответствующий многочлен Тома. 
Комбинируя члены в правой части равенств, мы получаем следующие рекуррентные соотношения, эквивалентные равенствам (3)-(4) теоремы:

$$
\begin{aligned}
& m_{\underline{\alpha}}=R_{\underline{\alpha}}+\sum_{1 \in J \subsetneq\{1, \ldots, r\}} R_{\underline{\alpha}_{J}} n_{\underline{\alpha}}, \\
& n_{\underline{\alpha}}=S_{\underline{\alpha}}+\sum_{1 \in J \subsetneq\{1, \ldots, r\}} S_{\underline{\alpha}_{J}} n_{\underline{\alpha}},
\end{aligned}
$$

где суммирование производится по всевозможньм собственньм подмножествам $J \subsetneq$ $\{1, \ldots, r\}$, содержащим элемент 1, и $\bar{J}=\{1, \ldots, r\} \backslash J$.

Например,

$$
\begin{aligned}
m_{\alpha} & =R_{\alpha}, \\
n_{\alpha}=s\left(m_{\alpha}\right) & =S_{\alpha}, \\
m_{\alpha, \beta} & =R_{\alpha, \beta}+R_{\alpha} S_{\beta}, \\
n_{\alpha, \beta}=s\left(m_{\alpha, \beta}\right) & =S_{\alpha, \beta}+S_{\alpha} S_{\beta}, \\
m_{\alpha, \beta, \gamma} & =R_{\alpha, \beta, \gamma}+R_{\alpha, \gamma} S_{\beta}+R_{\alpha, \beta} S_{\gamma}+R_{\alpha}\left(S_{\beta, \gamma}+S_{\beta} S_{\gamma}\right), \\
n_{\alpha, \beta, \gamma}=s\left(m_{\alpha, \beta, \gamma}\right) & =S_{\alpha, \beta, \gamma}+S_{\alpha, \gamma} S_{\beta}+S_{\alpha, \beta} S_{\gamma}+S_{\alpha} S_{\beta, \gamma}+S_{\alpha} S_{\beta} S_{\gamma} \\
& \text { и т. д. }
\end{aligned}
$$

3.3. ПримеР. Обозначим через $A_{r}$ класс особенностей, локальная алгебра которых изоморфна алгебре $\mathbb{C}[x] /\left\{x^{r+1}\right\}$ (т.е. класс особенностей, имеющих тип Бордмана $\Sigma^{1, \ldots, 1}, r$ вхождений). Тогда при $\ell=1$ мы имеем

$$
R_{A_{0}}=\bar{m}_{A_{0}}=1, \quad R_{A_{1}}=\bar{m}_{A_{1}}=c_{2}, \quad R_{A_{0}, A_{1}}=-2 c_{1} c_{2}-2 c_{3} .
$$

Классы когомологий, двойственные различньм циклам мультиособенностей, составленных из локальных особенностей $A_{0}, A_{1}$, следуюшие:

$$
\begin{aligned}
\bar{m}_{A_{0}, A_{1}} & =f^{*} f_{*}\left(c_{2}\right)-2 c_{1} c_{2}-2 c_{3}=s_{0,1}-2 c_{1} c_{2}-2 c_{3}, \\
\bar{m}_{A_{1}, A_{0}} & =c_{2} f^{*} f_{*}(1)-2 c_{1} c_{2}-2 c_{3}=c_{2} s_{0}-2 c_{1} c_{2}-2 c_{3}, \\
\bar{n}_{A_{0}, A_{1}} & =f_{*} \bar{m}_{A_{0}, A_{1}}=f_{*} \bar{m}_{A_{1}, A_{0}} \\
& =f_{*}(1) f_{*}\left(c_{2}\right)-2 f_{*}\left(c_{1} c_{2}+c_{3}\right) \\
& =s_{0} s_{0,1}-2 s_{1,1}-2 s_{0,0,1} .
\end{aligned}
$$

Обратим внимание, что соотношение (3) или (5) позволяет выразить $R_{\underline{\alpha}}$ по индукции через классы $m_{\underline{\alpha}}$ и $n_{\underline{\alpha}}=s\left(m_{\underline{\alpha}}\right)$ (доказательство см. ниже):

$$
\begin{aligned}
R_{\alpha}= & m_{\alpha}, \\
R_{\alpha, \beta}= & m_{\alpha, \beta}-m_{\alpha} n_{\beta}, \\
R_{\alpha, \beta, \gamma}= & m_{\alpha, \beta, \gamma}+2 m_{\alpha} n_{\beta} n_{\gamma}-m_{\alpha} n_{\beta, \gamma}-m_{\alpha, \beta} n_{\gamma}-m_{\alpha, \gamma} n_{\beta}, \\
& \cdots \\
R_{\underline{\alpha}}= & \sum_{J_{1} \sqcup \cdots \sqcup J_{k}=\{1, \ldots, r\}}(-1)^{k-1}(k-1) ! m_{\underline{\alpha}_{J_{1}}} n_{\underline{\alpha}_{J_{2}}} \cdots n_{\underline{\alpha}_{J_{k}}} .
\end{aligned}
$$


В этой форме теорема 3.2 утверждает, что для всякого собственного отображения $f: M \rightarrow N$ класс когомологий в $M$, заданньй комбинацией правой части равенства (6) от классов мультиособенностей $m_{\underline{\alpha}}=\# \operatorname{Aut}\left(\alpha_{2}, \ldots, \alpha_{r}\right)\left[p_{M}(M(\underline{\alpha}))\right]$ и $n_{\underline{\alpha}}=f^{*} f_{*} m_{\underline{\alpha}}$, выражается как универсальный многочлен от относительных классов Чженя $c_{i}(f)$.

Формула обрашения (8) является следствием следующего общего комбинаторного тождества. Рассмотрим семейство независимых коммутирующих переменных $Q_{J}$, занумерованных всевозможными непустыми подмножествами $J=\left\{j_{1}, \ldots, j_{|J|}\right\}$ заданного конечного множества индексов. Положим $Q_{J}^{(0)}=Q_{J}$ и

$$
Q_{J}^{(d)}=\sum_{J_{1} \sqcup \cdots \sqcup J_{k}=J} d^{k-1} Q_{J_{1}} \cdots Q_{J_{k}}, \quad d \in \mathbb{Z} .
$$

3.4. ЛеммА. При всех $d$ выполняется следующее тождество:

$$
Q_{J}^{(d)}=\sum_{J_{1} \sqcup \cdots \sqcup J_{k}=J}(d-1) \cdots(d-k+1) Q_{J_{1}}^{(1)} \cdots Q_{J_{k}}^{(1)} .
$$

В частности, при $d=0$ мы получаем формулу обрашения

$$
Q_{J}=\sum_{J_{1} \sqcup \cdots \sqcup J_{k}=J}(-1)^{k-1}(k-1) ! Q_{J_{1}}^{(1)} \cdots Q_{J_{k}}^{(1)} .
$$

ДокАЗАТЕЛЬСТво. Положим

$$
Q_{J}^{(d)}(k)=\sum_{J_{1} \sqcup \cdots \sqcup J_{k}=J} Q_{J_{1}}^{(d)} \cdots Q_{J_{k}}^{(d)}, \quad Q_{J}(k)=Q_{J}^{(0)}(k), \quad 1 \leqslant k \leqslant|J|,
$$

где суммирование проводится по всем разбиениям $J=J_{1} \sqcup \cdots \sqcup J_{k}$, в которых число $k$ подмножеств фиксировано. В частности,

$$
Q_{J}^{(1)}=\sum_{k=1}^{|J|} Q_{J}(k) .
$$

Тогда, раскрьв скобки и сгруппировав члены, мы получаем

$$
Q_{J}^{(1)}(k)=\sum_{J_{1} \sqcup \cdots \sqcup J_{l}=J} S(l, k) Q_{J_{1}} \cdots Q_{J_{l}}=\sum_{l=k}^{|J|} S(l, k) Q_{J}(k),
$$

где $S(l, k)$ - число Cтирлинга второго рода, т.е. число разбиений $l$-элементного множества в несвязное объединение $k$ непустых неупорядоченных подмножеств. Используя известное тождество (см. [11]) $\sum_{k=1}^{l}(d-1) \cdots(d-k+1) S(l, k)=d^{p-1}$, мы получаем окончательно

$$
\begin{aligned}
& \sum_{J_{1} \sqcup \cdots \sqcup J_{k}=J}(d-1) \cdots(d-k+1) Q_{J_{1}}^{(1)} \cdots Q_{J_{k}}^{(1)} \\
= & \sum_{k=1}^{|J|}(d-1) \cdots(d-k+1) Q_{J}^{(1)}(k) \\
= & \sum_{1 \leqslant k \leqslant l \leqslant|J|}(d-1) \cdots(d-k+1) S(l, k) Q_{J}(l) \\
= & \sum_{l=1}^{|J|} d^{l-1} Q_{J}(l)=Q_{J}^{(d)} .
\end{aligned}
$$


Лемма доказана.

Формула обрашения (8) следует из равенства (9), примененного к классам

$$
Q_{J}=\left\{\begin{array}{ll}
R_{\underline{\alpha}_{J}}, & 1 \in J, \\
S_{\underline{\alpha}_{J}}, & 1 \notin J,
\end{array} \quad Q_{J}^{(1)}=\left\{\begin{array}{ll}
m_{\underline{\alpha}_{J}}, & 1 \in J, \\
n_{\underline{\alpha}_{J}}, & 1 \notin J,
\end{array} \quad J \subset\{1, \ldots, r\},\right.\right.
$$

где $\underline{\alpha}=\left(\alpha_{1}, \ldots, \alpha_{r}\right)$ - фиксированный набор моноособенностей.

ДоКАЗАТЕЛЬСТВо ТЕОРЕМЫ 3.2. Поменяем временно определение остаточных многочленов $R_{\alpha}$. А именно, мы определим их индуктивно так, чтобы выполнялось равенство (3). Согласно лемме 3.4 , эквивалентным образом можно определить многочлены $R_{\underline{\alpha}}$ явной формулой (8). По теореме 2.2 правая часть равенства (8) является комбинацией многочленов от классов Чженя и классов Ландвебера-Новикова. Мы должны показать, что в этой комбинации все члены, содержашие классы $s_{I}$, сокрашаются.

Поскольку многочлены универсальны, соотношение (8) применимо для произвольных отображений. Мы будем применять его для последовательности отображений $f^{(d)}: M^{(d)} \rightarrow N, d=1,2, \ldots$, определенной следуюшим образом. Предположим, что исходное отображение $f: M \rightarrow N$ включается в семейство отображений из $M$ в $N$ такое, что для общих значений параметра семейства его члены находятся в общем положении по отношению друг к другу. Это предположение не является ограничительньм. Действительно, имеется достаточно большой запас примеров отображений, удовлетворяюших этому условию, так что всякое соотношение, полученное в этих предположениях, выполняется и в обшем случае. Возьмем в качестве $M^{(d)}=M_{1} \sqcup \cdots \sqcup M_{d}$, $M_{i} \cong M$, - несвязное объединение $d$ копий многообразия $M$, а в качестве ограничения $f_{i}: M_{i} \rightarrow N$ отображения $f^{(d)}$ на $i$-ю компоненту пространства $M^{(d)}$ - маленькое общее шевеление отображения $f$. Мы хотим применить равенство (8) к ограничениям классов мультиособенностей отображения $f^{(d)}$ на первую компоненту $M_{1} \cong M$ пространства $M^{(d)}$.

Поскольку каждое из отображений $f_{i}$ гомотопно исходному $f$, классы $n_{\underline{\alpha}}=$ $n_{\underline{\alpha}}\left(f_{i}\right)=n_{\underline{\alpha}}(f)$ не зависят от $i$. Рассмотрим на $N$ цикл мультиособенности $\underline{\alpha}=$ $\left(\alpha_{1}, \ldots, \alpha_{r}\right)$ отображения $f^{(d)}$. Для общей точки этого цикла $r$ ее прообразов могут быть распределены произвольным образом между компонентами пространства $M^{(d)}$. Поэтому для класса $\left.m_{\underline{\alpha}}^{(d)}\right|_{M_{1}}$ мы имеем очевидное соотношение

$$
\left.m_{\underline{\alpha}}^{(d)}\right|_{M_{1}}=\sum_{J_{1} \sqcup \ldots \sqcup J_{k}=\{1, \ldots, r\}}(d-1) \cdots(d-k+1) m_{\underline{\alpha}_{J_{1}}} n_{\underline{\alpha}_{J_{2}}} \cdots n_{\underline{\alpha}_{J_{k}}},
$$

где коэфффишиент $(d-1) \cdots(d-k+1)$ - количество способов выбрать $k-1$ различных упорядоченных элементов из заданного $(d-1)$-элементного множества $\left\{M_{2}, \ldots, M_{d}\right\}$ компонент пространства $M^{(d)}$, отличных от первой. Используя лемму 3.4 , это выражение можно переписать в виде

$$
\left.m_{\underline{\alpha}}^{(d)}\right|_{M_{1}}=\sum_{J_{1} \sqcup \ldots \sqcup J_{k}=\{1, \ldots, r\}} d^{k-1} R_{\underline{\alpha}_{J_{1}}} S_{\underline{\alpha}_{J_{2}}} \cdots S_{\underline{\alpha}_{J_{k}}} .
$$


Теперь вычислим классы Чженя-Ландвебера-Новикова отображения $f^{(d)}$. Ясно, что ограничение относительных классов Чженя на каждую компоненту пространства $M^{(d)}$ совпадает с соответствуюшими относительными классами Чженя отображения $f,\left.c_{k}\left(f^{(d)}\right)\right|_{M_{i}}=c_{k}(f)$. Следовательно, $s_{I}\left(f^{(d)}\right)=d s_{I}(f)$, т.е. переход от $f$ к $f^{(d)}$ действует на многочленах $m_{\underline{\alpha}}, n_{\underline{\alpha}}$ как однородное растяжение переменных $s_{I}$ с коэфофициентом $d$. Следовательно, если рассматривать $m_{\underline{\alpha}}$ как многочлен от $s_{I}$, то его свободный член совпадает со слагаемьм степени 0 по $d$ в выражении для $\left.m_{\underline{\alpha}}^{(d)}\right|_{M_{1}}$. Равенство (10) показывает, что это слагаемое равно в точности $R_{\underline{\alpha}}$. Это завершает доказательство равенства (3).

Равенство (4) вытекает из (3) применением гомоморфизма $f_{*}$ к обеим частям. Тот факт, что $R_{\alpha_{1}}, \ldots, \alpha_{r}$ не зависит от порядка вхождений $\alpha_{2}, \ldots, \alpha_{r}$, вытекает из симметричности явной формулы (8) (и очевидного замечания, что классы $n_{\underline{\alpha}}$ не зависят от порядка особенностей $\alpha_{i}$ ). Применив $f_{*}$ к обеим частям равенства $(\overline{8)}$, можно получить аналогичное выражение для $S_{\underline{\alpha}}$ :

$$
S_{\underline{\alpha}}=\sum_{J_{1} \sqcup \cdots \sqcup J_{k}=\{1, \ldots, r\}}(-1)^{k-1}(k-1) ! n_{\underline{\alpha}_{J_{1}}} \cdots n_{\underline{\alpha}_{J_{k}}} .
$$

Из этого равенства следует, что классы $S_{\underline{\alpha}}$ не зависят от порядка особенностей $\alpha_{i}$, а поскольку $R_{\underline{\alpha}}$ однозначно определяются по $S_{\underline{\alpha}}$, мы получаем, что и $R_{\underline{\alpha}}$ не зависят от порядка элементов во всем наборе $\underline{\alpha}=\left(\alpha_{1}, \ldots, \alpha_{r}\right)$. Теорема доказана.

3.5. ЗАмечАниЕ. Имеется вариант рассуждений, в котором не приходится шевелить отображение $f: M \rightarrow N$. А именно, рассмотрим отображение $f^{(d)}: M^{(d)} \rightarrow$ $N^{(d)}$, в котором в качестве $M^{(d)}$ берется $d$ экземпляров многообразий вида $M \times N^{d-1}$, в качестве $N^{(d)}=N^{d}$ - декартово произведение, а в качестве $f^{(d)}$ - отображение, которое на $i$-й компоненте $M \times N^{d-1}=N^{i-1} \times M \times N^{d-i}$ имеет вид $\mathrm{id}^{i-1} \times f \times$ $\mathrm{id}^{d-i}$. Мы построили отображение многообразий той же относительной размерности $\ell=\operatorname{dim} N^{(d)}-\operatorname{dim} M^{(d)}=\operatorname{dim} N-\operatorname{dim} M$. Кроме того, условие обшности положения для построенного отображения выполняется, если оно выполняется для исходного отображения $f$. Классы Чженя-Ландвебера-Новикова и классы мультиособенностей для отображения $f^{(d)}$ выражаются через соответствуюшие классы исходного отображения, что приводит к определенным соотношениям между ними. При вложении $\mathrm{id} \times f \times \cdots \times f: M \rightarrow M^{(d)}$, задаваемом мультиграфиком отображения $f$, эти соотношения сводятся к соотношениям, аналогичным (10), и те же рассуждения, что и вьше, приводят к формулам теоремы 3.2 .

\section{4. Пример: формула кратных особенностей}

Предположим, что тип мультиособенности $\underline{\alpha}=\alpha^{r}=(\alpha, \ldots, \alpha)$ образован $r$ одинаковыми локальными особенностями $\alpha$. Тогда, по теореме 3.2 , соответствуюшие клас-

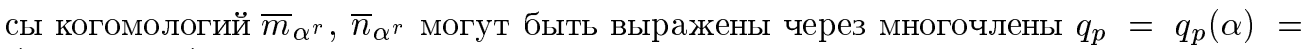
$\frac{1}{p !} R_{\alpha p+1}=\frac{1}{p !} R_{\alpha, \ldots, \alpha}(p+1$ вхождений) степени $(\ell+1) \operatorname{codim} \alpha+\ell p$. Например, если $\ell=1$ и $\alpha=\dot{A}_{0}$ (класс неособых ростков), то эти многочлены равны (см. п. 5)

$$
q_{0}=1, \quad q_{1}=-c_{1}, \quad q_{2}=c_{1}^{2}+c_{2}, \quad q_{3}=-c_{1}^{3}-3 c_{1} c_{2}-2 c_{3}, \ldots
$$


В случае $\ell=0, \alpha=A_{1}$ (особенность складки) этими многочленами являются (см. п. 5)

$$
q_{0}=c_{1}, \quad q_{1}=-2\left(2 c_{1}^{2}+c_{2}\right), \quad q_{2}=4\left(5 c_{1}^{3}+7 c_{1} c_{2}+3 c_{3}\right), \ldots
$$

Положим $v_{i}=v_{i}(\alpha)=\frac{1}{i} f_{*}\left(q_{i-1}\right)$ и определим формальные степенные ряды

$$
\begin{aligned}
m(t) & =\bar{m}_{\alpha}+\bar{m}_{\alpha^{2}} t+\bar{m}_{\alpha^{3}} t^{2}+\cdots \in \mathscr{C} \otimes \mathscr{S} \mathscr{C} \otimes \mathbb{Q}[[t]], \\
q(t) & =q_{0}+q_{1} t+q_{2} t^{2}+\cdots \in \mathscr{C} \otimes \mathbb{Q}[[t]], \\
n(t) & =1+\bar{n}_{\alpha} t+\bar{n}_{\alpha^{2}} t^{2}+\cdots \in \mathscr{S} \mathscr{C} \otimes \mathbb{Q}[[t]], \\
v(t) & =v_{1} t+v_{2} t^{2}+v_{3} t^{3}+\cdots \in \mathscr{S} \mathscr{C} \otimes \mathbb{Q}[[t]] ;
\end{aligned}
$$

тогда соотношения (4) и (6) принимают вид следующих двух эквивалентных друг другу равенств

$$
n(t)=\exp (v(t)) \quad \text { и } n^{\prime}(t)=v^{\prime}(t) n(t) \quad\left({ }^{\prime}=d / d t\right)
$$

T.e.

$$
\bar{n}_{\alpha}=v_{1}, \quad 2 \bar{n}_{\alpha^{2}}=2 v_{2}+v_{1}^{2}, \quad 6 \bar{n}_{\alpha^{3}}=6 v_{3}+6 v_{1} v_{2}+v_{1}^{3}, \ldots
$$

(эти полиномиальные выражения для $\bar{n}_{\alpha i}$ через $v_{i}$ известны, с точностью до перенормировки, как многочлены Белла). Действительно, каждое слагаемое в правой части равенства (6) имеет вид $S_{\underline{\alpha}_{J}} n_{\underline{\alpha}_{J}}=k ! v_{k}(n-k) ! \bar{n}_{\alpha^{r-k}}$, где $k$ - количество элементов в множестве $J \subset\{1, \ldots, r\}$. Количество аналогичных слагаемых с фиксированным $k$ равно $C_{r-1}^{k-1}$. Отсюда

$$
n_{\alpha^{r}}=r ! \bar{n}_{\alpha^{r}}=\sum_{k=1}^{r} C_{r-1}^{k-1} k !(r-k) ! v_{k} \bar{n}_{\alpha^{r-k}}, \quad \text { или } \quad r \bar{n}_{\alpha^{r}}=\sum_{k=1}^{r} k v_{k} \bar{n}_{\alpha^{r-k}} .
$$

В терминах производяших функций последнее соотношение записьвается в виде $\left(\bar{n}_{\alpha}+2 \bar{n}_{\alpha^{2}} t+\cdots\right)=\left(v_{1}+2 v_{2} t+\cdots\right)\left(1+\bar{n}_{\alpha} t+\bar{n}_{\alpha^{2}} t^{2}+\cdots\right)$ или $n^{\prime}(t)=v^{\prime}(t) n(t)$, как и утверждается.

"Интегрирование" этого равенства приводит к соотношению $n(t)=\exp (v(t))$. Последнее равенство можно получить и непосредственно из (4). Действительно, если $\underline{\alpha}=\alpha^{r}$, то каждый член в правой части (4) имеет вид

$$
S_{\underline{\alpha}_{J_{1}}} \cdots S_{\underline{\alpha}_{J_{k}}}=m_{1} ! \cdots m_{k} ! v_{m_{1}} \cdots v_{m_{k}} \text {, где } m_{i}=\left|J_{i}\right| \text {. }
$$

Количество таких слагаемых с фиксированными $k, m_{1}, \ldots, m_{k}$ равно

$$
\frac{r !}{\# \operatorname{Aut}\left(m_{1}, \ldots, m_{k}\right) m_{1} ! \cdots m_{k} !}
$$

где \# $\operatorname{Aut}\left(m_{1}, \ldots, m_{k}\right)$ - порядок подгрупшы перестановок из $k$ элементов, сохраняюших мультииндекс $\left(m_{1}, \ldots, m_{k}\right)$. Таким образом, $\bar{n}_{r}=n_{r} / r$ ! представляется в виде линейной комбинации различных мономов вида $v_{m_{1}} \cdots v_{m_{k}}$ и коэффициент, с которым входит этот моном в сумму, равен $1 / \# \operatorname{Aut}\left(m_{1}, \ldots, m_{k}\right)$.

Вычислим теперь коэффициент при $t^{k}$ в ряду

$$
\exp (v(t))=1+v(t)+v^{2}(t) / 2 !+\cdots .
$$


Этот коэффищиент также представляется в виде линейной комбинации мономов $v_{m_{1}} \cdots v_{m_{k}}$. Заметим, что мономы (однородной) степени $k$ могут появиться только из слагаемого $v^{k}(t) / k$ !. Более того, коэффициент при мономе $v_{m_{1}} \cdots v_{m_{k}}$ равен \# $\operatorname{Perm}\left(m_{1}, \ldots, m_{k}\right) / k$ !, где \# $\operatorname{Perm}\left(m_{1}, \ldots, m_{k}\right)$ - количество различных последовательностей вида $\left(m_{\sigma_{1}}, \ldots, m_{\sigma_{k}}\right)$, получаемых всевозможными перестановками набора $\left(m_{1}, \ldots, m_{k}\right)$. Применив очевидное комбинаторное соотношение

$$
\# \operatorname{Perm}\left(m_{1}, \ldots, m_{k}\right)=\frac{k !}{\# \operatorname{Aut}\left(m_{1}, \ldots, m_{k}\right)},
$$

мы получаем доказьваемое равенство $n(t)=\exp (v(t))$.

Выражения для $\bar{m}_{\alpha^{i}}$ получаются аналогичным образом. Из (5) вытекает соотношение $m(t)=q(t) n(t)$, которое можно переписать в виде рекуррентной формуль для классов кратной особенности $\alpha$ :

$$
\bar{m}_{\alpha^{r}}=\sum_{i=0}^{r-1} q_{i} \bar{n}_{\alpha^{r-1-i}}, \quad \bar{n}_{\alpha^{r}}=r f_{*}\left(\bar{m}_{\alpha^{r}}\right) .
$$

Заметим, что если $\alpha=A_{0}$ - класс неособого отображения, то $q_{0}=q_{0}\left(A_{0}\right)=1$, ряд $q(t)$ обратим и последнее соотношение в случае классов кратных точек $m_{r}=m_{A_{0}^{r}}$,

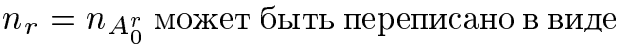

$$
n(t)=p(t) m(t), \quad p(t)=1+p_{1} t+p_{2} t^{2}+\cdots=\frac{1}{q(t)} \in \mathscr{C} \otimes \mathbb{Q}[[t]],
$$

или

$$
\bar{m}_{r}=f^{*}\left(\bar{n}_{r-1}\right)-\sum_{i=1}^{r-1} p_{i} \bar{m}_{r-i} .
$$

Существование многочленов $p_{r}$ от относительных классов Чженя $c_{i}\left(f^{*} T N-T M\right)$, удовлетворяюших соотношениям (11), было доказано в статьях Клеймана [30], [31] и Каца [21] в предположении, что отображение $f$ не имеет особенностей коранга, большего 1 (или когда этими особенностями можно пренебречь по соображениям размерности). Римани [47] показал, что при более удачном выборе многочленов $p_{6}, p_{7}$ (при $\ell=1)$ равенство (11) справедливо при $r \leqslant 7$ для любых устойчивых отображений. Мы утверждаем, что равенство (11) имеет место для любого собственного отображения и для любых $\ell, r \geqslant 1$. Более того, многочлены $p_{r}$ определяются этим условием однозначно.

\section{5. Определение остаточных многочленов $R_{\underline{\alpha}}$}

В этом пункте мы приводим алгоритм для вычисления остаточных многочленов $R_{\underline{\alpha}}$. Самый простой способ их вычислить для какой-либо конкретной мультиособенности $\underline{\alpha}$-применить метод, предложенньй Р. Римани для близкой задачи, см. [46], [47]. Этот метод основан на априорном сушествовании многочлена $R_{\underline{\alpha}}$, обеспечиваемом теоремой 3.2. А именно, мы определяем $R_{\underline{\alpha}}$ индуктивно как единственный многочлен, удовлетворяюший соотношению

$$
R_{\underline{\alpha}}=m_{\underline{\alpha}}-\sum_{J_{1} \sqcup \cdots \sqcup J_{l}=\{1, \ldots, r\}} R_{\underline{\alpha}_{J_{1}}} S_{\underline{\alpha}_{J_{2}}} \cdots S_{\underline{\alpha}_{J_{l}}} .
$$


Чтобы вычислить коэффициенты этого многочлена, мы рассматриваем набор примеров, в которых мы можем вычислить как классы Чженя-Ландвебера-Новикова, так и классы, двойственные циклам (мульти)особенностей. Каждый такой пример задает соотношения на коэффициенты многочлена $R_{\underline{\alpha}}$, и при достаточно разумном выборе примеров соотношения определяют многочлен однозначно.

Пусть задана группа Ли $H$, действующая линейно в пространствах $\mathbb{C}^{m}$ и $\mathbb{C}^{n}, n=$ $m+\ell$. Пусть $f_{0}: \mathbb{C}^{m} \rightarrow \mathbb{C}^{n}$ - собственное $H$-эквивариантное голоморфное отображение. Тогда в качестве $M$ и $N$ можно взять тотальные пространства векторных расслоений $\mathbb{C}_{H}^{n}, \mathbb{C}_{H}^{m}$ над классифицируюшим пространством группы $H$ (или его гладкой конечномерной аппроксимацией), ассоциированные с действием групшы $H$ в $\mathbb{C}^{m}, \mathbb{C}^{n}$ соответственно, и в качестве отображения $f: M \rightarrow N$-расслоенное отображение, совпадаюшее с $f_{0}$ на каждом слое. Тог да для этого конкретного отображения мы имеem:

(i) оба многообразия $M, N$ гомотопически эквивалентны $B H$, следовательно, мы можем отождествить когомологии пространств $M$ и $N$ и рассматривать все классы Чженя-Ландвебера-Новикова, а также классы мультиособенностей $m_{\underline{\alpha}}, n_{\underline{\alpha}}$ как элементы одного и того же кольца $H^{*}(M)=H^{*}(N)=H^{*}(B H)$ характеристических классов группы $H$;

(ii) относительньй класс Чженя равен $c(f)=c\left(\mathbb{C}_{H}^{n}\right) / c\left(\mathbb{C}_{H}^{m}\right)$, где $c\left(\mathbb{C}_{H}^{m}\right)$ и $c\left(\mathbb{C}_{H}^{n}\right)$ полные классы Чженя представлений группы $H$ в $\mathbb{C}^{m}$ и $\mathbb{C}^{n}$ соответственно; эти классы обычно легко вычисляются при помоши принципа расшепления;

(iii) классы Ландвебера-Новикова имеют вид

$$
s_{I}(f)=f_{*}\left(c^{I}(f)\right)=c^{I}(f) f_{*}(1),
$$

где $f_{*}(1)$ определяется из соотношения $c_{n}\left(\mathbb{C}_{H}^{n}\right)=c_{m}\left(\mathbb{C}_{H}^{m}\right) f_{*}(1)$ (это соотношение вытекает из коммутативности диаграммы

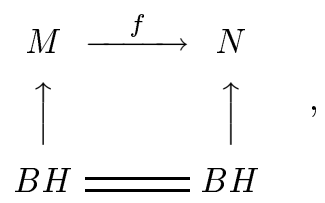

в которой вертикальные стрелки - вложения нулевого сечения, индуцируюшие в когомологиях умножение на старший класс Чженя соответствующего расслоения);

(iv) если $f_{0}$ имеет в начале координат особенность $\beta$, коразмерность которой не вьше коразмерности мультиособенности $\underline{\alpha}$, то $M(\underline{\alpha})$ пусто и $\bar{m}_{\underline{\alpha}}=0$, за исключением единственного случая, когда $r=1$ и $\underline{\alpha}=\{\beta\}$, и тогда $M(\underline{\alpha})=$ $M(\beta) \subset M-$ нулевое сечение расслоения $M \rightarrow B H$ и двойственньй класс когомологий $\bar{m}_{\underline{\alpha}}=\bar{m}_{\beta}=c_{m}\left(\mathbb{C}_{H}^{m}\right)$ - старший класс Чженя этого расслоения.

В качестве отображения $f_{0}: \mathbb{C}^{m} \rightarrow \mathbb{C}^{n}$ берется "устойчивая нормальная форма" одной из особенностей, определяемая как развертка миниверсальной деформации любого ее представителя вида $\mathbb{C}^{c} \rightarrow \mathbb{C}^{c+\ell}$, где $c$ - коранг особенности. В качестве $H$ берется группа симметрий этой особенности (см. [56], [46]). Для стабильной $\mathscr{K}$-классификашии група симметрий определяется следуюшим образом. 
Пусть $Q$ - локальная алгебра. Ее корангом назьвается минимальное количество образующих, или, что равносильно, минимальное число переменных $c$, такое, что эта алгебра реализуется как локальная алгебра некоторого ростка $\mathbb{C}^{c} \rightarrow \mathbb{C}^{c+\ell}$. Если алгебра порождена $m$ элементами, то минимальное необходимое число соотношений равно $m+d$, где $d$ не зависит от $m$ и назьвается дефектом алгебры.

Пусть $Q=Q(\alpha)$ - локальная алгебра, отвечающая данному классу $\alpha$ стабильной $\mathscr{K}$-эквивалентности. Выберем произвольного представителя $f:\left(\mathbb{C}^{c}, 0\right) \rightarrow\left(\mathbb{C}^{c+\ell}, 0\right)$ данного класса, где $c$ - коранг алгебры $Q$. Выберем произвольное достаточно большое $K$ и рассмотрим группу Ли $K$-струй $\mathscr{K}$-эквивалентностей.

5.1. ОПРЕДЕЛЕНИЕ. Группой симметрий $G_{\alpha}$ класса $\alpha$ называется максимальная компактная подгрупша в стабилизаторе $K$-струи ростка $f$.

Група симметрий не зависит, с точностью до изоморфизма, ни от представителя $f$, ни от выбора $K$, если $K$ достаточно велико. Ее явное описание дает следуюшее предложение.

5.2. ПРЕДЛОЖЕНИЕ. Группа симметрий особенности а изоморфна прямому произведению:

$$
G_{\alpha} \simeq \operatorname{Aut} Q \times U(\ell-d),
$$

где Aut $Q$ - максимальная компактная подгруппа в группе автоморфизмов алгебры $Q$.

ДокАЗАТЕЛЬСТво. Зададим алгебру $Q$ при помощи $c$ образуюших, где $c$ - ее коранг, и $c+\ell$ соотношений $f_{1}, \ldots, f_{c+\ell}$, последние $\ell-d$ из которых, $f_{c+d+1}, \ldots, f_{c+\ell}$, тождественно равны нулю. Поскольку всякое преобразование из $G_{\alpha}$ сохраняет идеал, порожденньй функциями $f_{i}$, имеется естественный гомоморфизм $G_{\alpha} \rightarrow \operatorname{Aut}(Q)$. Ясно, что всякое преобразование из ядра этого гомоморфизма сохраняет каждую из функций $f_{1}, \ldots, f_{c+d}$, а на функциях $f_{c+d+1}, \ldots, f_{c+\ell}$ действует всевозможными линейными заменами. С другой стороны, групу автоморфизмов алгебры $Q$ можно реализовать при помоши преобразований, в которых задействованы лишш функции $f_{1}, \ldots, f_{c+\ell}$. Таким образом, подгрупшы, участвуюшие в разложении теоремы, коммутируют, что завершает ее доказательство.

5.3. ЗАмЕчАниЕ. Отметим несколько соображений, облегчающих применение метода Римани. Первое замечание состоит в том, что группа симметрий $G_{\alpha}$ может быть заменена подгруппой $H \subset G_{\alpha}$ такой, что индуцированный гомоморфизм $H^{*}\left(B G_{\alpha}\right) \rightarrow$ $H^{*}(B H)$ инъективен. В частности, $G_{\alpha}$ может быть несвязной и в качестве $H$ можно взять компоненту единицы. Действительно, в этом случае ядро гомоморфизма $H^{*}\left(B G_{\alpha}\right) \rightarrow H^{*}(B H)$ состоит из элементов конечного порядка, так что замена $G_{\alpha}$ на $H$ не препятствует вычислению остаточного многочлена с рациональными коэффициентами. Но поскольку априорно известно, что в кольце характеристических классов векторных расслоений $H^{*}(B U)$ кручение отсутствует, найденный многочлен будет иметь только целые коэффициенты и будет представлять класс когомологий с целыми коэффициентами.

Второе замечание состоит в том, что система линейных уравнений на коэффициенты многочленов Тома, получаюшаяся применением этого метода, является чрезвычайно переопределенной. Чтобы исключить избыточные соотношения, можно воспользоваться следующим соображением. 
Предположим, что некоторый многочлен $P$ от относительных классов Чженя обращается в нуль, если отображение $f$ имеет только особенности коразмерности меньше $k$. Тогда для всякой особенности $\alpha$ коразмерности $k$ ограничение $P$ на соответствующее пространство расслоения над $B G_{\alpha}$ имеет виде $P^{\prime}$, где е - класс

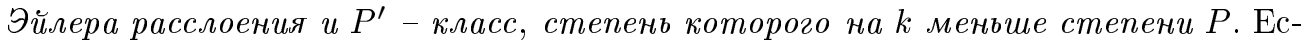
ли некоторые из коэффициентов многочлена $P$ неизвестны, то соотношения следует применять к классу $P^{\prime}=P /$ меньшей степени.

5.4. ЗАмЕчАниЕ. Больше соотношений можно получить, рассматривая нормальные формы мультиособенностей. Эти дополнительные соотношения позволяют непосредственно получить многочлены $m_{\underline{\alpha}}, n_{\underline{\alpha}}$ от классов Чженя-Ландвебера-Новикова без использования формул теоремы 3.2 . Однако в этом нет необходимости, поскольку теорема 3.2 сводит вычисление классов мультиособенностей к вычислению остаточных многочленов $R_{\underline{\alpha}}$.

5.5. ПРимеР. Пусть $\ell=1$. Рассмотрим в качестве "пробного отображения" $f: M \rightarrow N$ вложение нулевого сечения $M$ в тотальное пространство $N$ линейного расслоения с первым классом Чженя $t$. Тогда мы имеем $c_{1}(f)=t, c_{i}(f)=0$ при $i>1$, и $f^{*} f_{*}(1)=t$. Рассмотрения этого примера достаточно для определения коэффициента $а$ остаточного многочлена $R_{A_{0}^{2}}=a c_{1}$, входящего в формулу двойных точек $m_{A_{0}^{2}}=f^{*} f_{*}(1)+R_{A_{0}^{2}}$ : единственным возможным выбором для этого коэффициента является $a=-1$, т.е.

$$
m_{A_{0}^{2}}=f^{*} f_{*}(1)-c_{1}
$$

Этот пример показьвает преимущество метода: чтобы получить форму для класса двойных точек, справедливую для произвольного общего отображения, достаточно проверить ее на гладких вложениях.

Для мультиособенностей других типов рассмотрение данного примера приводит к следующему выводу: если тип мультиособенности $\underline{\alpha}$ содержит по крайней мере одну особенность, отличную от $A_{0}$, то коэффициент при $c_{1}^{\operatorname{codim} \underline{\alpha}}$ в остаточном многочлене $R_{\underline{\alpha}}$ обращается в нуль.

5.6. ПРимеР. Пусть $\ell=1$. Рассмотрим в качестве "пробного отображения" зонтик Кэли-Уитни - особенность, задаваемую отображением

$$
y_{1}=x_{1}^{2}, \quad y_{2}=x_{1} x_{2}, \quad y_{3}=x_{2}
$$

Эти формулы могут рассматриваться либо как отображение $\mathbb{C}^{2} \rightarrow\left[f_{0}\right] \mathbb{C}^{3}$ аффинных координатных пространств, либо как отображение $\xi \oplus \eta \rightarrow[f] \xi^{2} \oplus \xi \eta \oplus \eta$ тотальных пространств векторных расслоений $M \rightarrow B$ и $N \rightarrow B$ рангов 2 и 3 соответственно над некоторой базой $B$, где $\xi$ и $\eta$-некоторые линейные расслоения на $B$ с первыми классами Чженя $t_{1} \in H^{2}(B)$ и $t_{2} \in H^{2}(B)$ соответственно. Тогда для этого конкретного примера мы имеем

$$
\begin{aligned}
1+c_{1}(f)+c_{2}(f)+c_{3}(f)+\cdots & =\frac{\left(1+2 t_{1}\right)\left(1+t_{1}+t_{2}\right)\left(1+t_{2}\right)}{\left(1+t_{1}\right)\left(1+t_{2}\right)} \\
& =1+\left(2 t_{1}+t_{2}\right)+t_{1} t_{2}-t_{1}^{2} t_{2}+\cdots
\end{aligned}
$$


и $f_{*}(1)=\frac{2 t_{1}\left(t_{1}+t_{2}\right) t_{2}}{t_{1} t_{2}}=2\left(t_{1}+t_{2}\right)$. Рассмотрения этого примера достаточно для вычисления коэффициентов $a_{i}$ остаточного многочлена $R_{A_{0} A_{1}}=a_{1} c_{1}^{3}+a_{2} c_{1} c_{2}+$ $a_{3} c_{3}$, исходя из известных многочленов Тома $R_{A_{0}}=1, R_{A_{1}}=c_{2}$. Действительно, в этом примере цикл $M\left(A_{0} A_{1}\right)$ пуст и, подставив вычисленные классы в равенство $0=m_{A_{0} A_{1}}=R_{A_{0}} f_{*}\left(R_{A_{1}}\right)+R_{A_{0} A_{1}}$, мы получаем соотношение

$$
8 a_{1} t_{1}^{3}+\left(2+12 a_{1}+2 a_{2}-a_{3}\right) t_{1}^{2} t_{2}+\left(2+6 a_{1}+a_{2}\right) t_{1} t_{2}^{2}+a_{1} t_{2}^{3}=0 .
$$

Приравняв нулю коэффициенты, мы находим $a_{1}=0, a_{2}=a_{3}=-2$, иными словами, $R_{A_{0} A_{1}}=-2 c_{1} c_{2}-2 c_{3}$.

Отметим, что если принять во внимание равенство $a_{1}=0$ предыдушего примера, то последнее равенство принимает более простой вид

$$
\left(\left(2+2 a_{2}-a_{3}\right) t_{1}+\left(2+a_{2}\right) t_{3}\right) t_{1} t_{2}=0,
$$

что согласуется с утверждением замечания 5.3.

Интересно сравнить простые вычисления, проведенные вьше, с вычислением класса $R_{A_{0} A_{1}}$ (для $\ell=1$ ), предлагаемым теорией пересечений. Обозначим через $Z=M\left(A_{1}\right) \subset M$ множество критических точек отображения $f$ и положим $X=$ $f(Z) \subset N$. Тогда класс $-R_{A_{0} A_{1}}$ определяется как остаточньй класс к $m_{A_{0}, A_{1}}=$ $\left[p_{M}\left(M\left(A_{0}, A_{1}\right)\right)\right]$ в произведении-пересечении $X \cdot M=f^{*}(X)$. Для упрощения вычислений мы используем следующее наблюдение: если $\Sigma \subset M$ - алгебраическое подмножество коразмерности $c$, то включение $M \backslash \Sigma \rightarrow M$ индуцирует изоморфизм в когомологиях до (вешественной) размерности $2(c-1)$. Следовательно, мы можем всегда отбросить, без потери обшности, все вырождения высокой коразмерности и предполагать при вычислении класса $-R_{A_{0} A_{1}}$, что отображение $f: M \rightarrow N$ не имеет мультиособенностей комплексной коразмерности, большей 3.

Таким образом, мы предполагаем, что $Z \subset M$ и $X \subset N$ - гладкие вложенные подмногообразия коразмерностей 2 и 3 соответственно и $f$ отображает $Z$ на $X$ изоморфно. Полньй прообраз $f^{-1}(X)$ имеет две непересекаюшиеся компоненты, $f^{-1}(X)=$ $p_{M}\left(M\left(A_{0}, A_{1}\right)\right) \sqcup W$, где схема $W$ имеет носитель на $Z$. Тогда, по основной формуле теории пересечений, остаточный класс $-R_{A_{0} A_{1}}=f^{*}(X)-\left[p_{M}\left(M\left(A_{0}, A_{1}\right)\right)\right]$ имеет вид

$$
-R_{A_{0} A_{1}}=\left\{f^{*} c\left(\nu_{X} N\right) \frown s(W, M)\right\}_{m-3},
$$

где $\nu_{X} N$ - нормальное расслоение подмногообразия $X \subset N$. Довольно легко определить $f^{*} \nu_{X} N=T N-T X=T N-T Z=\nu_{f}+\nu_{Z} M$. Остается вычислить класс Сегре $s(W, M)$. Носитель $W$ совпадает с $Z$, но, как схема, $W$ отлична от $Z$, и даже не является приведенной. Локальные вычисления показьвают, что схема $W$ имеет кратность 2 вдоль $Z$ и "выпирает" в направлении линейного расслоения $\varkappa$ вдоль $Z$, задаваемого ядрами дифференщиала отображения $f$. Отсюда следует, что эта схема регулярно вложена и ее нормальное расслоение равно $\nu_{W} M=\nu_{Z} M-\varkappa+2 \varkappa$ (см. подробности в [26]). Следовательно,

$$
f^{*} c\left(\nu_{X} N\right) \frown s(W, M)=c\left(\nu_{X} M-\nu_{W} M\right) \frown[W]=c\left(\nu_{f}+\varkappa-2 \varkappa\right) \frown 2[Z],
$$


откуда мы получаем промежуточный ответ

$$
-R_{A_{0} A_{1}}=2 c_{1}\left(\nu_{f}+\varkappa-2 \varkappa\right) \frown[Z]=2\left(c_{1}(f)-c_{1}(\varkappa)\right) \frown[Z] .
$$

Осталось вычислить классы $[Z]=m_{A_{1}}$ и $c_{1}(\varkappa) \frown[Z]$. Для этих классов имеется формула

$$
\eta_{*}\left(-c_{1}(\varkappa)\right)^{k}=c_{\ell+k}(f),
$$

где $\eta: Z \rightarrow M-$ вложение множества критических значений (см., например, [20]). Применив эту формулу, мы получаем $[Z]=c_{2}(f)$ и $c_{1}(\varkappa) \frown[Z]=-c_{3}(f)$ соответственно, откуда, окончательно,

$$
-R_{A_{0} A_{1}}=2\left(c_{1}(f)-c_{1}(\varkappa)\right) \frown[Z]=2\left(c_{1}(f) c_{2}(f)+c_{3}(f)\right) .
$$

Отметим, что тот факт, что класс $R_{A_{0} A_{1}}$ выражается через относительные классы Чженя $c_{i}(f)$ (равно как и равенство $R_{A_{0} A_{1}}=R_{A_{1} A_{0}}$ ), получается при этом подходе как "неожиданньй” результат длинных вычислений.

Классы мультиособенностей и остаточные многочлены для $\ell=0$. При некоторых оговорках равенство теоремы 3.2 вьполняется и в случае $\ell=0$, т.е. для собственных отображений $f: M \rightarrow N$ многообразий одинаковой размерности, $\operatorname{dim} M=$ $\operatorname{dim} N$. Если $N$ связно, то корректно определена степень отображения $f$,

$$
d=f_{*}(1) \in H^{0}(N)=\mathbb{Z} .
$$

Пусть задан некоторый тип мультиособенности $\underline{\alpha}=\left(\alpha_{1}, \ldots, \alpha_{p}\right)$ такой, что $\operatorname{codim} \alpha_{i}>0$. Рассмотрим соответствуюший цикл мультиособенностей $p_{N}(M(\underline{\alpha})) \subset$ $N$ в многообразии области значений отображения. Полный прообраз всякой общей точки $y$ этого цикла состоит из $p$ точек $x_{1}, \ldots, x_{p}$, в которых $f$ имеет особенности $\alpha_{1}, \ldots, \alpha_{p}$, и еше $d-\mu$ точек, в которых $f$ имеет особенность $A_{0}$ (точки локального диффеоморфизма), где $\mu=\mu_{1}+\cdots+\mu_{p}$ и $\mu_{i}-$ локальная кратность отображения $f$ в $x_{i}$, определенная как размерность локальной алгебры ростка $f$ в точке $x_{i}$. Отсюда следует, что классы мультиособенностей $\left(\underline{\alpha}, A_{0}^{r}\right)=\left(\alpha_{1}, \ldots, \alpha_{p}, A_{0}, \ldots, A_{0}\right)$ (с $r$ вхождениями $A_{0}$ ) задаются равенством

$$
\begin{aligned}
& \bar{m}_{\underline{\alpha}, A_{0}^{r}}=C_{d-\mu}^{r} \bar{m}_{\underline{\alpha}}, \\
& m_{\underline{\alpha}, A_{0}^{r}}=(d-\mu)(d-\mu-1) \cdots(d-\mu-r+1) m_{\underline{\alpha}} .
\end{aligned}
$$

5.7. ПРЕДЛОЖЕНИЕ. Остаточные многочлены $R_{\underline{\alpha}}, A_{0}^{r}$ набора особенностей, содержащего классы $A_{0}$ локального диффеоморфизма, определяются остаточнымм многочленом $R_{\underline{\alpha}}$ остальных особенностей,

$$
R_{\underline{\alpha}, A_{0}^{r}}=(-1)^{(r-1)} \mu(\mu+1) \cdots(\mu+r-1) R_{\underline{\alpha}},
$$

где $\mu=\mu(\underline{\alpha})$ - сумма локальных кратностей особенностей, образующих набор $\underline{\alpha}$.

Доказательство предложения комбинаторное, оно состоит в проверке тождества (3) теоремы 3.2 для класса мультиособенности $\left(\alpha_{1}, \ldots, \alpha_{p}, A_{0}, \ldots, A_{0}\right)$. Заметим, что равенство (12) справедливо также, если набор $\underline{\alpha}$ содержит особенности $A_{0}$, т.е. вычисления достаточно проводить только для случая $r=1$, что совсем несложно. 
5.8. ПримеР. Числа, выражающие "классы кратных точек"

$$
\begin{aligned}
\bar{m}_{r} & =\bar{m}_{A_{0}^{r}}=C_{d-1}^{r-1} \in H^{0}(M)=\mathbb{Z} \\
\bar{n}_{r} & =\bar{n}_{A_{0}^{r}}=C_{d}^{r} \in H^{0}(N)=\mathbb{Z}
\end{aligned}
$$

выражаются через "остаточные классы" $q_{r}=\frac{1}{r !} R_{A_{0}^{r+1}}=(-1)^{r}$ по формулам п. 4 . В частности, мы получаем

$$
p(t)=\left(1+q_{1} t+q_{2} t^{2}+\cdots\right)^{-1}=1+t,
$$

и "формула кратных точек” в случае $\ell=0$ принимает вид равенства

$$
\bar{m}_{r+1}=f^{*}\left(\bar{n}_{r}\right)-\bar{m}_{r} \in H^{0}(M)=\mathbb{Z}
$$

которое эквивалентно очевидному тождеству $C_{d-1}^{r}=C_{d}^{r}-C_{d-1}^{r-1}$.

Предложение сводит задачу вычисления остаточных многочленов $R_{\underline{\alpha}}$ к случаю, когда все локальные особенности набора $\underline{\alpha}=\left(\alpha_{1}, \ldots, \alpha_{r}\right)$ имеют положительную коразмерность.

Список особенностей коразмерности, не превьшающей 8 , приведен в следующей таблице (см. [39], [46]). Эта таблища содержит также дополнительную информацию об особенностях, необходимую для вычисления остаточных многочленов.

\begin{tabular}{|c|c|c|c|c|}
\hline$\alpha$ & $Q_{\alpha}$ & $\operatorname{codim}$ & $H^{*}\left(B G_{\alpha}, \mathbb{Q}\right)$ & $c$ \\
\hline \hline$A_{p}, p \geqslant 0$ & $\frac{\mathbb{C}[x]}{\left\{x^{p+1}\right\}}$ & $p$ & $\mathbb{Q}[t]$ & $\frac{1+(p+1) t}{1+t}$ \\
\hline$I_{2,2}$ & $\frac{\mathbb{C}[x, y]}{\left\{x^{2}, y^{2}\right\}}$ & 4 & $\begin{array}{c}\mathbb{Q}[t, u] \\
\operatorname{deg} u=2\end{array}$ & $\frac{1+2 t+4 u}{1+t+u}$ \\
\hline $\begin{array}{c}I_{p, q}, 2 \leqslant p \leqslant q, \\
p+q>4\end{array}$ & $\frac{\mathbb{C}[x, y]}{\left\{x y, x^{p}+y^{q}\right\}}$ & $p+q$ & $\mathbb{Q}[t]$ & $\frac{(1+(p+q) t)(1+p q t)}{(1+p t)(1+q t)}$ \\
\hline & $\frac{\mathbb{C}[x, y]}{\left\{x^{2}, y^{3}\right\}}$ & 7 & $\mathbb{Q}\left[t, t_{1}\right]$ & $\frac{(1+2 t)\left(1+3 t_{1}\right)}{(1+t)\left(1+t_{1}\right)}$ \\
\hline & $\frac{\mathbb{C}[x, y]}{\left\{x^{2}+y^{3}, x y^{2}\right\}}$ & 8 & $\mathbb{Q}[t]$ & $\frac{(1+6 t)(1+7 t)}{(1+2 t)(1+3 t)}$ \\
\hline
\end{tabular}

Полный список соответствующих остаточных многочленов мультиособенностей можно найти в [28]. Этот список довольно обширен, и здесь мы его не приводим полностью. Ниже приведены остаточные многочлены особенностей вплоть до коразмерности 4, а также соответствующие им выражения для классов мультиособенностей. 
Остаточные классы $R_{\underline{\alpha}}$ коразмерности не выше 4 для $\ell=0$

$$
\begin{aligned}
R_{A_{1}} & =c_{1} \\
R_{A_{2}} & =c_{1}^{2}+c_{2} \\
R_{A_{1}^{2}} & =-2\left(2 c_{1}^{2}+c_{2}\right) \\
R_{A_{3}} & =c_{1}^{3}+3 c_{1} c_{2}+2 c_{3} \\
R_{A_{1} A_{2}} & =-6\left(c_{1}^{3}+2 c_{1} c_{2}+c_{3}\right) \\
R_{A_{1}^{3}} & =8\left(5 c_{1}^{3}+7 c_{1} c_{2}+3 c_{3}\right) \\
R_{A_{4}} & =c_{1}^{4}+6 c_{1}^{2} c_{2}+2 c_{2}^{2}+9 c_{1} c_{3}+6 c_{4} \\
R_{I_{22}} & =c_{2}^{2}-c_{1} c_{3} \\
R_{A_{1} A_{3}} & =-4\left(2 c_{1}^{4}+9 c_{1}^{2} c_{2}+2 c_{2}^{2}+11 c_{1} c_{3}+6 c_{4}\right) \\
R_{A_{2}^{2}} & =-3\left(3 c_{1}^{4}+12 c_{1}^{2} c_{2}+4 c_{2}^{2}+13 c_{1} c_{3}+8 c_{4}\right) \\
R_{A_{1}^{2} A_{2}} & =24\left(3 c_{1}^{4}+10 c_{1}^{2} c_{2}+2 c_{2}^{2}+10 c_{1} c_{3}+5 c_{4}\right) \\
R_{A_{1}^{4}} & =-48\left(14 c_{1}^{4}+37 c_{1}^{2} c_{2}+6 c_{2}^{2}+33 c_{1} c_{3}+15 c_{4}\right)
\end{aligned}
$$

Классы мультиособенностей $m_{\alpha_{1}, \underline{\alpha}^{\prime}}=\# \operatorname{Aut}\left(\underline{\alpha}^{\prime}\right) \bar{m}_{\alpha_{1}, \underline{\alpha}^{\prime}}$ коразмерности не выше 4 для $\ell=0$

$$
\begin{aligned}
m_{A_{1}, A_{1}}= & s_{1} c_{1}-4 c_{1}^{2}-2 c_{2} \\
m_{A_{1}, A_{2}}= & s_{2} c_{1}+s_{01} c_{1}-6 c_{1}^{3}-12 c_{1} c_{2}-6 c_{3} \\
m_{A_{2}, A_{1}}= & s_{1} c_{1}^{2}-6 c_{1}^{3}+s_{1} c_{2}-12 c_{1} c_{2}-6 c_{3} \\
m_{A_{1}, A_{1}^{2}}= & s_{1}^{2} c_{1}-4 s_{2} c_{1}-2 s_{01} c_{1}-8 s_{1} c_{1}^{2}+40 c_{1}^{3}-4 s_{1} c_{2}+56 c_{1} c_{2}+24 c_{3} \\
m_{A_{1}, A_{3}}= & s_{3} c_{1}+3 s_{11} c_{1}+2 s_{001} c_{1}-8 c_{1}^{4}-36 c_{1}^{2} c_{2}-8 c_{2}^{2}-44 c_{1} c_{3}-24 c_{4} \\
m_{A_{2}, A_{2}}= & s_{2} c_{1}^{2}+s_{01} c_{1}^{2}-9 c_{1}^{4}+s_{2} c_{2}+s_{01} c_{2}-36 c_{1}^{2} c_{2}-12 c_{2}^{2}-39 c_{1} c_{3}-24 c_{4} \\
m_{A_{3}, A_{1}}= & s_{1} c_{1}^{3}-8 c_{1}^{4}+3 s_{1} c_{1} c_{2}-36 c_{1}^{2} c_{2}-8 c_{2}^{2}+2 s_{1} c_{3}-44 c_{1} c_{3}-24 c_{4} \\
m_{A_{1}, A_{1} A_{2}=}= & s_{1} s_{2} c_{1}-6 s_{3} c_{1}+s_{1} s_{01} c_{1}-12 s_{11} c_{1}-6 s_{001} c_{1}-4 s_{2} c_{1}^{2}-4 s_{01} c_{1}^{2} \\
& -6 s_{1} c_{1}^{3}+72 c_{1}^{4}-2 s_{2} c_{2}-2 s_{01} c_{2}-12 s_{1} c_{1} c_{2}+240 c_{1}^{2} c_{2}+48 c_{2}^{2} \\
& -6 s_{1} c_{3}+240 c_{1} c_{3}+120 c_{4} \\
m_{A_{2}, A_{1}^{2}}= & s_{1}^{2} c_{1}^{2}-4 s_{2} c_{1}^{2}-2 s_{01} c_{1}^{2}-12 s_{1} c_{1}^{3}+72 c_{1}^{4}+s_{1}^{2} c_{2}-4 s_{2} c_{2}-2 s_{01} c_{2} \\
& -24 s_{1} c_{1} c_{2}+240 c_{1}^{2} c_{2}+48 c_{2}^{2}-12 s_{1} c_{3}+240 c_{1} c_{3}+120 c_{4} \\
m_{A_{1}, A_{1}^{3}=} & s_{1}^{3} c_{1}-12 s_{1} s_{2} c_{1}+40 s_{3} c_{1}-6 s_{1} s_{01} c_{1}+56 s_{11} c_{1}+24 s_{001} c_{1}-12 s_{1}^{2} c_{1}^{2} \\
& +48 s_{2} c_{1}^{2}+24 s_{01} c_{1}^{2}+120 s_{1} c_{1}^{3}-672 c_{1}^{4}-6 s_{1}^{2} c_{2}+24 s_{2} c_{2}+12 s_{01} c_{2} \\
& +168 s_{1} c_{1} c_{2}-1776 c_{1}^{2} c_{2}-288 c_{2}^{2}+72 s_{1} c_{3}-1584 c_{1} c_{3}-720 c_{4}
\end{aligned}
$$


Классы мультиособенностей $n_{\underline{\alpha}}=\# \operatorname{Aut}(\underline{\alpha}) \bar{n}_{\underline{\alpha}}$ коразмерности не выше 4 для $\ell=0$

$$
\begin{aligned}
n_{A_{1}}= & s_{1} \\
n_{A_{2}}= & s_{2}+s_{01} \\
n_{A_{1}^{2}}= & s_{1}^{2}-4 s_{2}-2 s_{01} \\
n_{A_{3}}= & s_{3}+3 s_{11}+2 s_{001} \\
n_{A_{1} A_{2}}= & s_{1} s_{2}-6 s_{3}+s_{1} s_{01}-12 s_{11}-6 s_{001} \\
n_{A_{1}^{3}}= & s_{1}^{3}-12 s_{1} s_{2}+40 s_{3}-6 s_{1} s_{01}+56 s_{11}+24 s_{001} \\
n_{A_{4}}= & s_{4}+2 s_{02}+6 s_{21}+9 s_{101}+6 s_{0001} \\
n_{I_{22}=}= & s_{02}-s_{101} \\
n_{A_{1} A_{3}=}= & s_{1} s_{3}-8 s_{4}-8 s_{02}+3 s_{1} s_{11}-36 s_{21}+2 s_{1} s_{001}-44 s_{101}-24 s_{0001} \\
n_{A_{2}^{2}}= & s_{2}^{2}-9 s_{4}+2 s_{2} s_{01}+s_{01}^{2}-12 s_{02}-36 s_{21}-39 s_{101}-24 s_{0001} \\
n_{A_{1}^{2} A_{2}=}= & s_{1}^{2} s_{2}-4 s_{2}^{2}-12 s_{1} s_{3}+72 s_{4}+s_{1}^{2} s_{01}-6 s_{2} s_{01}-2 s_{01}^{2}+48 s_{02}-24 s_{1} s_{11} \\
& +240 s_{21}-12 s_{1} s_{001}+240 s_{101}+120 s_{0001} \\
n_{A_{1}^{4}=} & s_{1}^{4}-24 s_{1}^{2} s_{2}+48 s_{2}^{2}+160 s_{1} s_{3}-672 s_{4}-12 s_{1}^{2} s_{01}+48 s_{2} s_{01}+12 s_{01}^{2} \\
& -288 s_{02}+224 s_{1} s_{11}-1776 s_{21}+96 s_{1} s_{001}-1584 s_{101}-720 s_{0001}
\end{aligned}
$$

Классы мультиособенностей и остаточные многочлены для $\ell=1$. Следующая таблица содержит список классов локальных особенностей ростков отображений $\left(\mathbb{C}^{m}, 0\right) \rightarrow\left(\mathbb{C}^{m+1}, 0\right)$ вплоть до коразмерности 8 вместе с разнообразной дополнительной информацией, необходимой для вычисления остаточных многочленов.

\begin{tabular}{|c|c|c|c|c|}
\hline$\alpha$ & $Q_{\alpha}$ & codim & $H^{*}\left(B G_{\alpha}, \mathbb{Q}\right)$ & $c$ \\
\hline \hline$A_{0}$ & 1 & 0 & $\mathbb{Q}[t]$ & $1+t$ \\
\hline$A_{k}$ & $\frac{\mathbb{C}[x]}{\left\{x^{k+1}\right\}}$ & $2 k$ & $\mathbb{Q}\left[t_{1}, t_{2}\right]$ & $\frac{\left(1+t_{1}\right)\left(1+(k+1) t_{2}\right)}{1+t_{2}}$ \\
\hline$I I I_{2,2}$ & $\frac{\mathbb{C}[x, y]}{\left\{x^{2}, y^{2}, x y\right\}}$ & 6 & $\begin{array}{c}\mathbb{Q}[t, u] \\
\operatorname{deg} u=2\end{array}$ & $\frac{(1+t)(1+2 t+4 u)}{(1+t+u)}$ \\
\hline$I_{2,2}$ & $\frac{\mathbb{C}[x, y]}{\left\{x^{2}, y^{2}\right\}}$ & 7 & $\begin{array}{c}\mathbb{Q}\left[t, t_{1}, u\right] \\
\operatorname{deg} u=2\end{array}$ & $\frac{\left(1+t_{1}\right)(1+2 t+4 u)}{(1+t+u)}$ \\
\hline$I I I_{2,3}$ & $\frac{\mathbb{C}[x, y]}{\left\{x^{2}, y^{3}, x y\right\}}$ & 8 & $\mathbb{Q}\left[t, t_{1}\right]$ & $\frac{(1+2 t)\left(1+t+t_{1}\right)\left(1+3 t_{1}\right)}{(1+t)\left(1+t_{1}\right)}$ \\
\hline
\end{tabular}


Остаточные многочлены малой степени и выражения для соответствующих классов мультиособенностей приведены ниже. Полньй список вычисленных многочленов имеется в [28].

Остаточные классы $R_{\underline{\alpha}}$ коразмерности не выше 5 для $\ell=1$

$$
\begin{aligned}
R_{A_{0}} & =1 \\
R_{A_{0}^{2}} & =-c_{1} \\
R_{A_{1}} & =c_{2} \\
R_{A_{0}^{3}} & =2\left(c_{1}^{2}+c_{2}\right) \\
R_{A_{0} A_{1}} & =-2\left(c_{1} c_{2}+c_{3}\right) \\
R_{A_{0}^{4}} & =-6\left(c_{1}^{3}+3 c_{1} c_{2}+2 c_{3}\right) \\
R_{A_{2}} & =c_{2}^{2}+c_{1} c_{3}+2 c_{4} \\
R_{A_{0}^{2} A_{1}} & =2\left(3 c_{1}^{2} c_{2}+2 c_{2}^{2}+7 c_{1} c_{3}+6 c_{4}\right) \\
R_{A_{0}^{5}} & =24\left(c_{1}^{4}+6 c_{1}^{2} c_{2}+2 c_{2}^{2}+9 c_{1} c_{3}+6 c_{4}\right) \\
R_{A_{0} A_{2}} & =-3\left(c_{1} c_{2}^{2}+c_{1}^{2} c_{3}+2 c_{2} c_{3}+4 c_{1} c_{4}+4 c_{5}\right) \\
R_{A_{1}^{2}} & =-2\left(2 c_{1} c_{2}^{2}+c_{1}^{2} c_{3}+4 c_{2} c_{3}+5 c_{1} c_{4}+6 c_{5}\right) \\
R_{A_{0}^{3} A_{1}} & =-24\left(c_{1}^{3} c_{2}+2 c_{1} c_{2}^{2}+4 c_{1}^{2} c_{3}+3 c_{2} c_{3}+8 c_{1} c_{4}+6 c_{5}\right) \\
R_{A_{0}^{6}} & =-120\left(c_{1}^{5}+10 c_{1}^{3} c_{2}+10 c_{1} c_{2}^{2}+25 c_{1}^{2} c_{3}+12 c_{2} c_{3}+38 c_{1} c_{4}+24 c_{5}\right)
\end{aligned}
$$

Классы мультиособенностей $m_{\alpha_{1}, \underline{\alpha}^{\prime}}=\# \operatorname{Aut}\left(\underline{\alpha}^{\prime}\right) \bar{m}_{\alpha_{1}, \underline{\alpha}^{\prime}}$ коразмерности не выше 4 для $\ell=1$

$$
\begin{aligned}
m_{A_{0}, A_{0}}= & s_{0}-c_{1} \\
m_{A_{0}, A_{0}^{2}}= & s_{0}^{2}-s_{1}-2 s_{0} c_{1}+2 c_{1}^{2}+2 c_{2} \\
m_{A_{0}, A_{1}}= & s_{01}-2 c_{1} c_{2}-2 c_{3} \\
m_{A_{1}, A_{0}}= & s_{0} c_{2}-2 c_{1} c_{2}-2 c_{3} \\
m_{A_{0}, A_{0}^{3}}= & s_{0}^{3}-3 s_{0} s_{1}+2 s_{2}+2 s_{01}-3 s_{0}^{2} c_{1}+3 s_{1} c_{1}+6 s_{0} c_{1}^{2} \\
& -6 c_{1}^{3}+6 s_{0} c_{2}-18 c_{1} c_{2}-12 c_{3} \\
m_{A_{0}, A_{0} A_{1}=} & s_{0} s_{01}-2 s_{11}-2 s_{001}-s_{01} c_{1}-2 s_{0} c_{1} c_{2}+6 c_{1}^{2} c_{2}+4 c_{2}^{2}-2 s_{0} c_{3} \\
& +14 c_{1} c_{3}+12 c_{4} \\
m_{A_{1}, A_{0}^{2}=} & s_{0}^{2} c_{2}-s_{1} c_{2}-4 s_{0} c_{1} c_{2}+6 c_{1}^{2} c_{2}+4 c_{2}^{2}-4 s_{0} c_{3}+14 c_{1} c_{3}+12 c_{4} \\
m_{A_{0}, A_{0}^{4}=} & s_{0}^{4}-6 s_{0}^{2} s_{1}+3 s_{1}^{2}+8 s_{0} s_{2}-6 s_{3}+8 s_{0} s_{01}-18 s_{11}-12 s_{001}-4 s_{0}^{3} c_{1} \\
& +12 s_{0} s_{1} c_{1}-8 s_{2} c_{1}-8 s_{01} c_{1}+12 s_{0}^{2} c_{1}^{2}-12 s_{1} c_{1}^{2}-24 s_{0} c_{1}^{3}+24 c_{1}^{4} \\
& +12 s_{0}^{2} c_{2}-12 s_{1} c_{2}-72 s_{0} c_{1} c_{2}+144 c_{1}^{2} c_{2}+48 c_{2}^{2}-48 s_{0} c_{3} \\
& +216 c_{1} c_{3}+144 c_{4}
\end{aligned}
$$




\section{Классы мультиособенностей $n_{\underline{\alpha}}=\# \operatorname{Aut}(\underline{\alpha}) \bar{n}_{\underline{\alpha}}$ коразмерности не выше 5 для $\ell=1$}

$$
\begin{aligned}
n_{A_{0}}= & s_{0} \\
n_{A_{0}^{2}}= & s_{0}^{2}-s_{1} \\
n_{A_{1}}= & s_{01} \\
n_{A_{0}^{3}}= & s_{0}^{3}-3 s_{0} s_{1}+2 s_{2}+2 s_{01} \\
n_{A_{0} A_{1}}= & s_{0} s_{01}-2 s_{11}-2 s_{001} \\
n_{A_{0}^{4}}= & s_{0}^{4}-6 s_{0}^{2} s_{1}+3 s_{1}^{2}+8 s_{0} s_{2}-6 s_{3}+8 s_{0} s_{01}-18 s_{11}-12 s_{001} \\
n_{A_{2}}= & s_{02}+s_{101}+2 s_{0001} \\
n_{A_{0}^{2} A_{1}=} & s_{0}^{2} s_{01}-s_{1} s_{01}+4 s_{02}-4 s_{0} s_{11}+6 s_{21}-4 s_{0} s_{001}+14 s_{101}+12 s_{0001} \\
n_{A_{0}^{5}}= & s_{0}^{5}-10 s_{0}^{3} s_{1}+15 s_{0} s_{1}^{2}+20 s_{0}^{2} s_{2}-20 s_{1} s_{2}-30 s_{0} s_{3}+24 s_{4}+20 s_{0}^{2} s_{01} \\
& \quad-20 s_{1} s_{01}+48 s_{02}-90 s_{0} s_{11}+144 s_{21}-60 s_{0} s_{001}+216 s_{101}+144 s_{0001}
\end{aligned}
$$

\section{6. Обоснование из теории кобордизмов}

В этом пункте мы приводим топологические соображения, обосновывающие теорему 2.2. Приведенные соображения довольно схематичны, так что в полной мере теорему 2.2 нельзя считать доказанной. При достаточном упорстве эти соображения несложно довести до полной строгости, однако это вряд ли стоит делать: окончательное доказательство, равно как и более строгая формулировка, уточняющая понятия классов особенностей и обшности положения, должны формулироваться в рамках алгебраической геометрии и теории пересечений, см. обсуждение этих вопросов в п. 7. Поэтому последуюшие топологические аргументы следует рассматривать как (довольно убедительную) мотивировку всей теории.

Классифицирующее пространство моноособенностей. Напомним определение классифицируюшего пространства моноособенностей и доказательство сушествования многочленов Тома. Приводимая ниже конструкция эквивалентна конструкции, использованной в [22]-[24].

Рассмотрим классификацию особенностей стабильной $\mathscr{K}$-эквивалентности ростков отображений $\left(\mathbb{C}^{m}, 0\right) \rightarrow\left(\mathbb{C}^{m+\ell}, 0\right)$, где $\ell>0$ фиксировано, а $m$ может быть различньг (см. п. 1). С этой классификацией мы связьваем так назьваемое классифицируюшее пространство $B \mathscr{K}_{\ell}$. Топология этого пространства отражает теорию характеристических классов векторных расслоений, в то время как его геометрия отражает геометрию разбиения пространства струй отображений $\left(\mathbb{C}^{m}, 0\right) \rightarrow\left(\mathbb{C}^{m+\ell}, 0\right)$ на классы $\mathscr{K}$-эквивалентности. Пространство $B \mathscr{K}_{\ell}$ определяется как предел его конечномерных приближений $B \mathscr{K}_{\ell}(m, d, K)$ при $m, d, K \rightarrow \infty$. В действительности нет необходимости рассматривать бесконечномерные пространства. Если мы интересуемся характеристическими классами, связанными с особенностями коразмерности не вьше заданного конечного числа $c$, то достаточно зафиксировать достаточно большие $m, d, K$ и положить $B \mathscr{K}_{\ell}=B \mathscr{K}_{\ell}(m, d, K)$. 
Зафиксируем некоторые $K \gg 0, d \gg m \gg 0$. Рассмотрим комплексный грассманиан $G_{m+\ell, d}=G_{m+\ell, d}^{\mathbb{C}}$ как апшроксимацию для классифицирующего пространства $B U(m+\ell)$ комплексных векторных расслоений ранга $(m+\ell)$. Пусть $E_{m+\ell, d} \rightarrow$ $G_{m+\ell, d}$ - соответствующее классифицирующее векторное расслоение. Мы отождествляем $G_{m+\ell, d}$ с нулевым сечением $G_{m+\ell, d} \subset E_{m+\ell, d}$, рассматриваемым как подмногообразие в тотальном пространстве.

6.1. ОПРЕДЕЛЕНИЕ. Классифицирующим пространством В $\mathscr{K}_{\ell}(m, d, K) \mathscr{K}_{\text {-осо- }}$ бенностей называется пространство $K$-струй ростков отображений $f:\left(\mathbb{C}^{m}, 0\right) \rightarrow$ $\left(E_{m+\ell, d}, G_{m+\ell, d}\right)$.

С точностью до выбора из стягиваемого пространства, всякий росток $f$ определяется образом начала координат $f(0) \in G_{m+\ell, d}$. Следовательно, когомологии классифицируюшего пространства $H^{*}\left(B \mathscr{K}_{\ell}(m, d, K)\right)=H^{*}\left(G_{m+\ell, d}\right)$ стабилизируются при $m, d \rightarrow \infty$ к кольцу характеристических классов векторных расслоений, т.е. к кольцу многочленов от классов Чженя $c_{1}, c_{2}, \ldots$ С другой стороны, с каждым ростком $f:\left(\mathbb{C}^{m}, 0\right) \rightarrow\left(E_{m+\ell, d}, G_{m+\ell, d}\right)$ можно связать класс $\mathscr{K}$-особенности, а именно особенности пересечения образа отображения $f$ с нулевым сечением в пространстве $E_{m+\ell, d}$, или, более точно, локальной алгебры прообраза $f^{-1}\left(G_{m+\ell, d}\right)$. Класс изоморфизма этой алгебры определяется однозначно по $K$-струе отображения $f$, и если $K$ достаточно велико, то коразмерность пространства ростков, локальные алгебры которых не определяются по $K$-струе, стремится к бесконечности с ростом $K$. Таким образом, каждый класс $\mathscr{K}$-особенностей $\alpha$ задает (гладкое полуалгебраическое незамкнутое) подмногообразие $B \alpha \subset B \mathscr{K}_{\ell}$.

6.2. ОПРЕДЕЛЕНИЕ. Многочленом Тома класса особенностей $\alpha$ называется полиномиальное выражение через образуюшие $c_{1}, c_{2}, \ldots$ для класса когомологий $[\overline{B \alpha}] \in$ $H^{*}\left(B \mathscr{K}_{\ell}\right)=H^{*}(B U)$, двойственного по Пуанкаре замыканию многообразия $B \alpha$.

Для доказательства существования многочлена Тома теоремы 1.2 мы по всякому гладкому отображению $f: M \rightarrow N, \operatorname{dim} N-\operatorname{dim} M=\ell$, строим классифицируюшее отображение $\varkappa: M \rightarrow B \mathscr{K}_{\ell}$. Классифицируюшее свойство этого отображения заключается в том, что разбиение многообразия $M$ на страты $\mathscr{K}$-особенностей отображения $f$ индуцировано отображением $\varkappa$ из соответствующего разбиения пространства $B \mathscr{K}_{\ell}$.

Мы предлагаем здесь две конструкции для отображения $\varkappa$. Первая конструкция является глобальной (ср. [22], [23]), в то время как вторая использует подробное изучение локальных особенностей (ср. [48]). В действительности, обе конструкции эквивалентны, поскольку $\varkappa$ определено однозначно с точностью до гомотопии (это нетрудно вьвести из любой из двух конструкций). Из первой конструкции вытекает, что

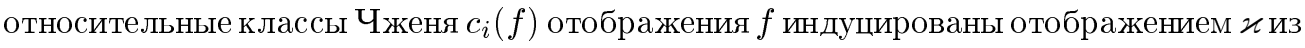
образуюших $c_{i} \in H^{*}\left(B \mathscr{K}_{\ell}\right) \simeq H^{*}(B U)$. Это завершает доказательство теоремы 1.2.

6.3. ЗАмЕчАниЕ. Приведенное вьше определение многочленов Тома, по сушеству, алгебраическое; его легко приспособить к произвольному алгебраически замкнутому основному полю и к кольцам Чжоу вместо когомологий. С другой стороны, классифицирующее отображение $\varkappa$ не является голоморфным. Мы можем добиться только того, чтобы оно было $C^{\infty}$-гладким. Поэтому приведенное доказательство существования многочленов Тома существенно топологическое. Насколько мне извест- 
но, доказательство теоремы 1.2, которое использовало бы лишь методы алгебраической геометрии, никогда не было опубликовано.

Первая конструкция классифицирующего отображения $\varkappa: M \rightarrow B \mathscr{K}_{\ell}$. Имеются гладкие "экспоненциальные” отображения $T M \rightarrow M, T N \rightarrow N$, определенные на трубчатой окрестности нулевого сечения в пространствах касательного расслоения, которые отображают изоморфно слой над каждой точкой на окрестность этой точки. Экспоненциальное отображение можно выбрать таким, чтобы его ограничение на слои было голоморфным, но глобально оно может быть сделано лишш $C^{\infty}$-гладким. Используя это отображение, можно поднять данное отображение $f: M \rightarrow N$ до расслоенного отображения пространств векторных расслоений $\widehat{f}: T M \rightarrow f^{*} T N$ таким образом, чтобы ограничение отображения $\widehat{f}$ на слои $T_{x} M \rightarrow T_{f(x)} N$ было голоморфным и имело тот же тип особенности в начале координат, что и росток $(M, x) \rightarrow(N, f(x))$ исходного отображения $f$ для каждой точки $x \in M$.

Для всякого вспомогательного комплексного векторного расслоения $F \rightarrow M$ можно рассмотреть расслоенное отображение пространств векторных расслоений $\widehat{f} \oplus \mathrm{id}: T M \oplus F \rightarrow f^{*} T N \oplus F$. Заметим, что ограничение отображения $\widehat{f} \oplus \mathrm{id}$ на каждый слой $T_{x} M \oplus F_{x} \rightarrow T_{f(x)} N \oplus F_{x}$ голоморфно и имеет $\mathscr{K}$-особенность в начале координат, стабильно эквивалентную особенности отображения $f$ в точке $x$.

Расслоение $F$ можно выбирать произвольно. Выберем его таким образом, чтобы $T M \oplus F$ было изоморфно тривиальному расслоению $\mathbb{C}^{m} \times M \rightarrow M$ (расслоение $F$ и тривиализация не являются обязательно голоморфными). В этом случае отображение $\widehat{f} \oplus$ id задает $C^{\infty}$-гладкое семейство голоморфных ростков $\left(\mathbb{C}^{m}, 0\right) \rightarrow$ $\left(T_{f(x)} N \oplus F_{x}, 0\right)$, параметризованное точками $x \in M$. В свою очередь, расслоение $f^{*} T N \oplus F$ ранга $m+\ell$ над $M$ индуцировано подходяшим классифицирующим отображением из классифицируюшего отображения над грассманианом $E_{m+\ell, d} \rightarrow G_{m+\ell, d}$, $d \gg 0$, что задает отображение тотальных пространств $f^{*} T N \oplus F \rightarrow E_{m+\ell, d}$. Взяв композицию построенных двух отображений, мы получаем желаемое отображение $\varkappa$. Согласно конструкции, классы $\varkappa^{*} c_{i}$ имеют вид

$$
\varkappa^{*} c_{i}=c_{i}\left(f^{*} T N \oplus F\right)=c_{i}\left(f^{*} T N-T M\right)=c_{i}(f) .
$$

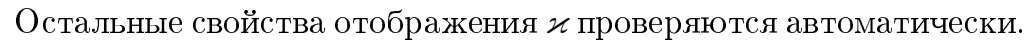

Вторая конструкция классифицирующего отображения $\varkappa: M \rightarrow B \mathscr{K}_{\ell}$ является вариантом обобщенной конструкции Понтрягина-Тома, использованной А. Сючем в некоторых близких задачах. Она основана на следующем наблюдении. Пусть $\alpha$ - класс особенностей, которьй состоит из единственного класса стабильной $\mathscr{K}$-эквивалентности (т.е. $\alpha$ является классом ростков, локальная алгебра которых изоморфна некоторой данной). Обозначим через $G_{\alpha}$ группу симметрий этого класса (см. п. 5).

6.4. Лемма. Многообразие В $\alpha \subset$ В $\mathscr{K}_{\ell}$ гомотопически әквивалентно классифичирующему пространству $B G_{\alpha}$ группь $G_{\alpha}$.

По-видимому, эта лемма не была нигде сформулирована именно в таком виде. Однако некоторые очень близкие формулировки можно найти в [22], и, поскольку доказательство в данном случае аналогично, здесь мы его не приводим. 
В конструкции классифицирующего пространства $\varkappa$ мы предполагаем, что отображение $f$ имеет лиш конечное число неэквивалентных особенностей и что струйное расширение отображения $f$ трансверсально разбиению пространства струй на классы особенностей (иными словами, все локальные особенности отображения $f$ устойчивые и простые). Тогда каждый цикл особенностей является гладким (возможно, незамкнутым) подмногообразием ожидаемой размерности. С использованием понятия клеточной классификации (см. [54], [23]) конструкцию можно без труда распространить на случай произвольных отображений.

Сперва выберем некоторьй цикл особенностей $M(\alpha) \subset M$ наименьшей возможной размерности. Он замкнут. Из определения групшы симметрий вытекает, что структурная группа нормального расслоения к $M(\alpha)$ редуцируется к $G_{\alpha}$. По приведенной лемме нормальное расслоение подмногообразия $M(\alpha)$ классифицируется непрерьвным отображением $M(\alpha) \rightarrow B \alpha$, которое определено однозначно с точностью до гомотопии, и это отображение продолжается до отображения трубчатой окрестности подмногообразия $M(\alpha)$ с сохранением разбиений пространств $M$ и $B \mathscr{K}_{\ell}$ на типы особенностей. Затем мы продолжаем отображение $\varkappa$ аналогичньм образом шаг за шагом на окрестности остальных стратов, сохраняя его в тех точках, где оно уже определено. Подробности этой конструкции (в несколько иной ситуации) можно найти в [48].

Классифицирующее пространство вещественных мультиособенностей. Рассмотрим гладкое собственное отображение общего положения $f: M \rightarrow N$ вещественных гладких многообразий, $\ell=\operatorname{dim}_{\mathbb{R}} N-\operatorname{dim}_{\mathbb{R}} M \geqslant 0$. Замькание цикла мультиособенностей $p_{N}(M(\underline{\alpha})) \subset N$ является локально полуалгебраическим подмножеством, и его двойственньй класс когомологий не всегда корректно определен (даже в когомологиях с коэффищиентами в $\mathbb{Z}_{2}$ ). Такие классы когомологий определены только для определенных линейных комбинаций мультиособенностей, удовлетворяющих некоторым граничным условиям (см. [54]). Пусть $\chi(f) \in H^{*}\left(N, \mathbb{Z}_{2}\right)$ - один из классов, определенньй таким способом.

Отображение $f$ может рассматриваться как представитель класса $\ell$-мерных кобордизмов на $N$. Легко увидеть, что класс $\chi$ является инвариантом класса кобордизма, другими словами, если два отображения $f: M \rightarrow N$ и $f^{\prime}: M^{\prime} \rightarrow N$ кобордантны, то $\chi(f)=\chi\left(f^{\prime}\right)$. Более того, имеет место следуюшее утверждение.

6.5. ПрЕДЛОЖЕнИЕ. Если $d$-мерный класс когомологий (для определенности, по модулю 2), двойственный некоторой линейной комбинации ииклов особенностей коразмерности d, корректно определен для всякого общего собственного отображсения $f: M \rightarrow N$ многообразий относительной размерности $\operatorname{dim} N-\operatorname{dim} M=\ell$, то он задает универсальную когомологическую операцию из $\ell$-мерных кобордизмов $O^{\ell}(\cdot)$ в $d$-мерныце когомологии $H^{d}\left(\cdot, \mathbb{Z}_{2}\right)$.

Классификация всех возможных операций (не обязательно являюшихся стабильными или аддитивными, но с фиксированньм $\ell$ ) может быть получена из классической конструкщии Тома [51]. Один из способов описания конструкшии Тома состоит в следуюшем. Имеется "универсальный $\ell$-мерньй кобордизм" $\mathbf{f}: \mathbf{M}_{\ell}^{\mathbb{R}} \rightarrow \mathbf{N}_{\ell}^{\mathbb{R}}$ такой, что всякий класс кобордизмов на всяком многообразии $N$ индуцируется из $\mathbf{f}$ некоторым непрерьвным отображением $\varkappa: N \rightarrow \mathbf{N}_{\ell}^{\mathbb{R}}$, определенным однозначно с точностью до гомотопии. В качестве пространства $\mathbf{N}_{\ell}^{\mathbb{R}}$ можно взять итерированное пространство 
петель $\Omega^{m} M O(m+\ell), m \gg 0$, где $M O(n)$ - пространство Тома классифицируюшего расслоения ранга $n$ над $B O(n)$ (пространства $B O(n), \mathbf{N}_{\ell}^{\mathbb{R}}$ всегда можно заменить их гладкими конечномерными аппроксимациями). Отображение $\varkappa: N \rightarrow \mathbf{N}_{\ell}^{\mathbb{R}}$ может рассматриваться как отображение $\tilde{\varkappa}: N \times S^{m} \rightarrow M O(m+\ell)$. Предположим, что это отображение гладко в окрестности нулевого сечения $B O(m+\ell) \subset M O(m+\ell)$ и трансверсально ему. Тогда мы можем положить $M=\tilde{\varkappa}^{-1}(B O(m+\ell))$, и $f: M \rightarrow N-$ естественная проекция. Всякое гладкое отображение $M \rightarrow N$ может быть реализовано этой конструкцией, и два отображения $f_{i}: M_{i} \rightarrow N, i=1,2$, кобордантны, если и только если соответствуюшие отображения $\varkappa_{i}: N \rightarrow \mathbf{N}_{\ell}^{\mathbb{R}}$ гомотопны (см., например, [51]). Применяя стандартные рассуждения, мы приходим к следующему результату.

6.6. ПРЕДЛОЖЕНИЕ. Элементы пространства всех возможниых когомологических операций $O^{\ell}(\cdot) \rightarrow H^{d}\left(\cdot, \mathbb{Z}_{2}\right)$ находятся во взаимно однозначном соответствии с классами когомологий группь $H^{d}\left(\Omega^{m} M O(m+\ell), \mathbb{Z}_{2}\right), m \gg 0$ (эти группы стабилизируются для достаточно больших т).

Как известно, пространства Тома $M O(m+\ell)$ в размерностях, не превосходящих $2(m+\ell)$, имеют гомотопический тип произведения пространств Эйленберга-Маклейна $K\left(\mathbb{Z}_{2}, n\right)$ для различных $n$. На пространствах Эйленберга-Маклейна операция перехода к пространству петель сводится к сдвигу числа $n$. Это приводит к явному описанию когомологий пространства $\Omega^{m} M O(m+\ell), m \gg 0$, а следовательно, возможных операций $O^{\ell}(\cdot) \rightarrow H^{d}\left(\cdot, \mathbb{Z}_{2}\right)$. Мы опускаем подробности, поскольку в дальнейшем нас будет интересовать, в основном, комплексньй случай.

6.7. ОПРЕДЕЛЕНИЕ. Классифицирующим пространством вещественных $\mathscr{K}$ мультиособенностей назьвается пространство $\mathbf{N}_{\ell}^{\mathbb{R}}=\Omega^{m} M O(m+\ell), m \gg 0$, или его конечномерная апшроксимация.

Элементами этого пространства являются непрерывные отображения $h:\left(S^{m}, *\right) \rightarrow$ $(M O(m+\ell), *)$, где * обозначает отмеченные точки в $S^{m}$ и $M O(m+\ell)$ соответственно. Без потери обшности, можно считать, что это отображение $h$ гладко в окрестности нулевого сечения $B O(m+\ell) \subset M O(m+\ell)$. Поскольку $\ell \geqslant 0$, то, за исключением подмножества бесконечной коразмерности, всякое такое отображение $h$ имеет конечное число прообразов нулевого сечения. Для каждой точки $x \in h^{-1}(B O(m+\ell))$ корректно определен ее тип $\mathscr{K}$-особенности (заданный как класс изоморфизма локальной алгебры 0-мерного особого многообразия $h^{-1}(B O(m+\ell))$ в точке $\left.x\right)$. Следовательно, точки классифицируюшего пространства классифищированы в соответствии с различными типами мультиособенностей.

6.8. Лемма. Если кажддий класс локальных особенностей $\alpha_{i}$, входящих в заданный тип мультиособенности $\underline{\alpha}=\left(\alpha_{1}, \ldots, \alpha_{r}\right)$, состоит из одной орбить $\mathscr{K}$-эквивалентности, то подпространство $\mathbf{N}(\underline{\alpha}) \subset \mathbf{N}_{\ell}^{\mathbb{R}}$ гомотопически эквивалентно классифицирующему пространству группы симметрий мультиособенности $\underline{\alpha}$.

Под группй симметрий мультиособенности $\underline{\alpha}$ мы понимаем полупрямое произведение групп симметрий индивидуальных особенностей $G_{\alpha_{1}} \times \cdots \times G_{\alpha_{r}}$ и подгрупшы автоморфизмов $\operatorname{Aut}\left(\alpha_{1}, \ldots, \alpha_{r}\right) \subset S(r)$ мультииндекса $\underline{\alpha}$. 
Лемма вытекает из аналогичной леммы 6.4 для случая локальных особенностей. Действительно, рассмотрим \# $\operatorname{Aut}(\underline{\alpha})$-листное накрытие $\widetilde{\mathbf{N}}(\underline{\alpha}) \rightarrow \mathbf{N}(\underline{\alpha})$, слои которого над точкой $h \in \mathbf{N}(\underline{\alpha})$ находятся во взаимно однозначном соответствии с различными упорядочиваниями точек из полного прообраза $h^{-1}(B O(m+\ell))$, в которых $h$ имеет эквивалентные локальные особенности. Пространство $\widetilde{\mathbf{N}}(\underline{\alpha})$ расслоено над конфигурационным пространством упорядоченных наборов из $r$ точек в $\mathbb{R}^{m}$, и по лемме 6.4 слои этого расслоения изоморфны $B G_{\alpha_{1}} \times \cdots \times B G_{\alpha_{r}}$.

Поскольку $m$ велико, конфигурационное пространство имеет тривиальную топологию (т.е. оно $k(m)$-связно, где $k(m)$ растет неограниченно с ростом $m$ ). Групша $\operatorname{Aut}(\underline{\alpha})$ действует на этом пространстве свободно, откуда и следует результат.

6.9. ЗАмечАниЕ. Конструкция Тома предоставляет классифицирующее отображение $\varkappa: N \rightarrow \mathbf{N}_{\ell}^{\mathbb{R}}$, ассоциированное с отображением $f: M \rightarrow N$. Отметим, что оно сохраняет разбиения $N$ и $\mathbf{N}_{\ell}^{\mathbb{R}}$ на страты различных типов мультиособенностей. Альтернативно, для построения классифицируюшего отображения $\varkappa$ можно использовать "обобщенную конструкцию Понтрягина-Тома" [48] (перенос которой со случая устойчивых отображений, рассмотренного в [48], на случай произвольных отображений представляет техническую, но не принципиальную трудность). Простое наблюдение об эквивалентности обеих конструкций отсутствует в [48].

6.10. ЗАмЕчАниЕ. Пространство $\mathbf{M}_{\ell}^{\mathbb{R}}$ области определения "универсального $\ell$ мерного кобордизма" $\mathbf{M}_{\ell}^{\mathbb{R}} \rightarrow \mathbf{N}_{\ell}^{\mathbb{R}}$ задается как подпространство прямого произведения $S^{m} \times \mathbf{N}_{\ell}^{\mathbb{R}}$, образованное парами вида $(x, h)$ такими, что $h(x) \in B O(m+\ell)$. Пространство $\mathbf{M}_{\ell}^{\mathbb{R}}$ также имеет естественное разбиение. Страты этого разбиения занумерованы наборами локальных особенностей $\underline{\alpha}=\left(\alpha_{1}, \ldots, \alpha_{r}\right)$, одна из которых, скажем, $\alpha_{1}$, отмечена. Эта классификация отражает классификацию классов мультиособенностей вида $p_{M}(M(\underline{\alpha}))$ на многообразии $M$ области определения гладкого отображения $M \rightarrow N$. Аналог леммы 6.8 также справедлив. Группа $\operatorname{Aut}\left(\alpha_{1}, \ldots, \alpha_{r}\right)$ должна быть заменена при этом на меньшую подгруппу $\operatorname{Aut}\left(\alpha_{2}, \ldots, \alpha_{r}\right)$, содержашую только те перестановки мультииндекса $\underline{\alpha}$, которые оставляют на месте отмеченный элемент $\alpha_{1}$.

Классифицирующее пространство комплексных мультиособенностей. Собственное голоморфное отображение $f: M \rightarrow N$ комплексных многообразий может рассматриваться как представитель унитарного $2 \ell$-мерного класса кобордизмов $[f] \in U^{2 \ell}(N)$, где $\ell=\operatorname{dim}_{\mathbb{C}} N-\operatorname{dim}_{\mathbb{C}} M$. Неверно, что каждый класс комплексных кобордизмов представляется голоморфным отображением. Тем не менее, можно ожидать, что, аналогично вешественному случаю, соответствие, сопоставляющее отображению $f: M \rightarrow N$ класс когомологий в образе $N$, двойственньй некоторому циклу мультиособенностей, продолжается до универсальной когомологической операции $U^{2 \ell}(\cdot) \rightarrow H^{*}(\cdot)$ (мы рассматриваем все операции, не обязательно являюшиеся стабильными или аддитивными, при этом число $\ell$ считается фиксированным). Предложение 6.14, сформулированное ниже, утверждает, что это действительно справедливо.

Рассуждения, аналогичные рассмотренным выше для вещественного случая, показывают, что возможные когомологические операции $U^{2 \ell}(\cdot) \rightarrow H^{*}(\cdot)$ находятся во взаимно однозначном соответствии с элементами кольца когомологий $H^{*}\left(\mathbf{N}_{\ell}^{\mathbb{C}}\right)$, где $\mathbf{N}_{\ell}^{\mathbb{C}}=\Omega^{2 m} M U(m+\ell), m \rightarrow \infty,-$ соответствуюшее итерированное пространство 
петель. Элементы пространства $\mathbf{N}_{\ell}^{\mathbb{C}}$ - непрерьвные отображения

$$
h:\left(S^{2 m}, *\right) \rightarrow(M U(m+\ell), *) .
$$

Обозначим через $\mathbf{M}_{\ell}^{\mathbb{C}} \subset S^{2 m} \times \mathbf{N}_{\ell}^{\mathbb{C}}$ подпространство, образованное парами вида $(x, h)$ такими, что $h(x) \in B U(m+\ell)$. Естественная проекция $\mathbf{f}: \mathbf{M}_{\ell}^{\mathbb{C}} \rightarrow \mathbf{N}_{\ell}^{\mathbb{C}}$ может рассматриваться как "универсальньй унитарный кобордизм".

Известно, что когомологии пространства $\Omega^{2 m} M U(m+\ell), m \gg 0$, не имеют кручения, тем не менее полное описание мультипликативной структуры в кольце целочисленных когомологий классифицирующего пространства довольно сложно, и здесь важную роль играют вопросы делимости, см. [10], [40], [45].

$\mathrm{K}$ счастью, ситуация сильно упрощается, если перейти к когомологиям с рациональными коэффициентами. Для всякого монома $c^{I}=c_{1}^{i_{1}} c_{2}^{i_{2}} \cdots$ соответствуюший ему класс Ландвебера-Новикова $s_{I}$ не зависит от отображения, представляющего данньй класс кобордизмов. Это дает большой запас искомых операций. Операции Ландвебера-Новикова хорошо известны в теории кобордизмов. В действительности, они определены в кобордизмах, здесь же мы рассматриваем их образы в когомологиях. Из операций Ландвебера-Новикова можно получать новые, переходя к произведению (произведение рассматривается в смысле кольцевой структуры в когомологиях, а не в смысле взятия композиции операций).

6.11. ТЕОРемА. Для всякого $\ell>0$ кольца когомологий $H^{*}\left(\mathbf{N}_{\ell}^{\mathbb{C}}, \mathbb{Q}\right)$ и $H^{*}\left(\mathbf{M}_{\ell}^{\mathbb{C}}, \mathbb{Q}\right)$ представляют собой кольца многочленов, образующими которьх служат абстрактные классы Ландвебера-Новикова $s_{I}$ и Чженя-Ландвебера-Новикова $c_{i}$, $s_{\text {I }}$ соответственно.

6.12. СЛЕДСТВИЕ. Для всякого $\ell>0$ имеется взаимно однозначное соответствие межсду операциями $U^{2 \ell}(\cdot) \rightarrow H^{*}(\cdot, \mathbb{Q})$ и әлементами кольиа многочленов от классов Ландвебера-Новикова $s_{I}$.

ДокАЗАТЕЛЬСТво. Как известно, стабильньй остов пространства $M U(m+\ell)$ при $m \rightarrow \infty$ имеет рациональный гомотопический тип произведения пространств Эйленберга-Маклейна $K(\mathbb{Z}, 2 n)$ для различных $n$. Более подробно, зафиксируем базис $c_{I}$ в группе когомологий пространства $B U(m+\ell)$. Например, в качестве такого базиса можно взять всевозможные мономы от классов Чженя тавтологического векторного расслоения ранга $m+\ell$. Обозначим через $\varphi_{I} \in H^{*}(M U(m+\ell))$ образ класса $c_{I}$ при изоморфизме Тома $H^{*}(B U(m+\ell)) \simeq H^{*}(M U(m+\ell))$. Класс $\varphi_{I}$ задает, в свою очередь, отображение $M U(m+\ell) \rightarrow K(\mathbb{Z}, 2(m+\ell+|I|))$, где $|I|-$ степень монома $c_{I}$ относительно градуировки, в которой $\operatorname{deg} c_{i}=i$. Переходя к произведению, мы получаем отображение

$$
M U(m+\ell) \longrightarrow \prod K(\mathbb{Z}, 2(m+\ell+|I|))
$$

Из теоремы Картана-Серра вытекает, что это отображение индуцирует изоморфизм в рациональньх когомологиях в степенях $2(m+\ell)+d$ при $m \gg d$, см., например, [41]. Переходя к пространствам петель, мы получаем отображение

$$
\Omega^{2 m} M U(m+\ell) \longrightarrow \prod K(\mathbb{Z}, 2(\ell+|I|)),
$$


которое также индуцирует изоморфизм в $d$-мерных рациональных когомологиях при фиксированном $d$ и достаточно большом $m$. Поскольку $2(\ell+|I|)$ четно, кольцо $H^{*}(K(\mathbb{Z}, 2(\ell+|I|)), \mathbb{Q})$ изоморфно кольцу многочленов от одной образующей $s_{I}$, в точности соответствуюшей операции Ландвебера-Новикова. Отсюда вытекает описание кольца когомологий пространства $H^{*}\left(\mathbf{N}_{\ell}^{\mathbb{C}}, \mathbb{Q}\right)=\Omega^{2 m} M U(m+\ell)$. Описание целочисленного кольца когомологий классифицирующего пространства сложнее. В [10] ответ формулируется в терминах характера Чженя-Дольда.

Структура рациональных когомологий пространства $\mathbf{M}_{\ell}^{\mathbb{C}}$ вычисляется аналогично. Элементы этого пространства образованы отображениями сферы $S^{2 m}$ с двумя отмеченньми точками в пространство $M U(m+\ell)$ такими, что первая отмеченная точка переходит в отмеченную точку пространства Тома, а вторая - в одну из точек нулевого сечения $B U(m+\ell) \subset M U(m+\ell)$. Отсюда следует, что $\mathbf{M}_{\ell}^{\mathbb{C}}$ расслоено над $B U(m+\ell)$, а когомологии слоя в стабильных размерностях не отличаются от когомологий пространства $\mathbf{N}_{\ell}^{\mathbb{C}}$. Таким образом, из рассмотрения спектральной последовательности этого расслоения мы получаем искомое равенство $H^{*}\left(\mathbf{M}_{\ell}^{\mathbb{C}}\right) \simeq H^{*}(B U) \otimes H^{*}\left(\mathbf{N}_{\ell}^{\mathbb{C}}\right)$. Теорема 6.11 полностью доказана.

Рассмотрим сферу $S^{2 m}$ как одноточечную компактификацию комплексного пространства $\mathbb{C}^{m}$. Тогда пространства областей определения и значения отображения $h:\left(S^{2 m}, *\right) \rightarrow(M U(m+\ell), *)$, представляющего элемент $h \in \mathbf{N}_{\ell}^{\mathbb{C}}$, являются гладкими комплексньми многообразиями всюду, за исключением отмеченных точек. Обозначим через $\mathbf{N}_{\ell}^{\text {hol }} \subset \mathbf{N}_{\ell}^{\mathbb{C}}$ подпространство, содержашее только такие отображения $h$, которые голоморфны в окрестности нулевого сечения $B U(m+l) \subset M U(m+\ell)$, и положим $\mathbf{M}_{\ell}^{\mathrm{hol}}=\mathbf{f}^{-1}\left(\mathbf{N}_{\ell}^{\mathrm{hol}}\right) \subset \mathbf{M}_{\ell}^{\mathbb{C}}$.

6.13. ОПРЕДЕЛЕНИЕ. Пространства $\mathbf{N}_{\ell}^{\text {hol }}, \mathbf{M}_{\ell}^{\text {hol }}, \ell>0$, назьваются классифииирующими пространствами комплексных $\mathscr{K}$-мультиособенностей.

6.14. ПРЕДЛОЖЕНИЕ. Для всякого класса мультиособенностей $\underline{\alpha}=\left(\alpha_{1}, \ldots\right.$, $\alpha_{r}$ ) соответствие $f \mapsto \bar{n}_{\underline{\alpha}} \in H^{*}(N)$, заданное на собственных голоморфных отображениях $f: M \rightarrow N$, продолжается до универсальной когомологической операции $U^{2 \ell}(\cdot) \rightarrow H^{*}(\cdot, \mathbb{Q})$, и, следовательно, $\bar{n}_{\underline{\alpha}}$ может быть выражено как универсальный многочлен с рациональными коэффициентами от классов Ландвебера-Новикова.

ДОКАЗАТЕЛЬСТВО ПРЕДЛОЖЕНИЯ 6.14 И ТЕОРЕМЫ 2.2. Можно Показать, что вложение $\mathbf{N}_{\ell}^{\text {hol }} \subset \mathbf{N}_{\ell}^{\mathbb{C}}$ индуцирует изоморфизм когомологий. Это утверждение является одним из проявлений общего принщипа Смейла-Хирша и может быть доказано методами, изложенными в книге [55]. Точки пространства $\mathbf{N}_{\ell}^{\text {hol }}$ классифицированы по типам мультиособенностей голоморфных отображений. Аналогично вешественному случаю, подпространство $\mathbf{N}(\underline{\alpha}) \subset \mathbf{N}_{\ell}^{\text {hol }}$, соответствуюшее фиксированному типу мультиособенностей $\underline{\alpha}$, гомотопически эквивалентно классифищирующему пространству $B G_{\underline{\alpha}}$ группы симметрий $G_{\underline{\alpha}}$ особенности $\underline{\alpha}$. По теореме 6.11 класс когомологий в $\mathbf{N}_{\ell}^{\text {hol }}$, дВойственньй по Пуанкаре замыканию цикла $\mathbf{N}(\underline{\alpha})$, является многочленом с рациональньми коэффициентами от классов Ландвебера-Новикова. 
Если $f: M \rightarrow N$ - собственное голоморфное отображение, то, используя обобщенную конструкцию Понтрягина-Тома, изложенную в [48], можно построить классифицирующее отображение $\varkappa: N \rightarrow \mathbf{N}_{\ell}^{\text {hol }}$, которое сохраняет разбиение на типы мультиособенностей. Следовательно, класс когомологий $n_{\underline{\alpha}} \in H^{*}(N)$, соответствующий мультиособенности $\underline{\alpha}$, индуцирован отображением $\varkappa$ из класса $[\mathbf{N}(\underline{\alpha})] \in H^{*}\left(\mathbf{N}_{\ell}^{\mathrm{hol}}\right)$. Композиция $\varkappa$ с вложением $\mathbf{N}_{\ell}^{\text {hol }} \subset \Omega^{2 m} M U(m+\ell)$ - отображение, предоставляемое классической конструкцией Тома. Следовательно, образующие $s_{I} \in H^{*}\left(\mathbf{N}_{\ell}^{\text {hol }}\right)$ переводятся гомоморфизмом $\varkappa^{*}$ в соответствуюшие классы Ландвебера-Новикова, ассоциированные с отображением $f$. Это доказьвает предложение.

Теорема 2.2 следует из подобных рассуждений, примененных к пространству $\mathbf{M}_{\ell}^{\text {hol }}$ вместо $\mathbf{N}_{\ell}^{\text {hol }}$.

6.15. ЗАмЕчАниЕ. Рассуждения этого пункта послужили начальной точкой наших исследований мультиособенностей. Удостоверившись в сушествовании многочленов предложения 6.14, мы вычислили их явно для большого количества мультиособенностей при помоши метода, предложенного Р. Римани для близкой задачи. Затем мы обнаружили между этими многочленами много интересных соотношений. Например, степень (однородная) многочлена равна числу индивидуальных особенностей, образующих данную мультиособенность; старшая однородная компонента равна, с точностью до множителя \# Aut $(\underline{\alpha})$, произведению классов, соответствуюших моноособенностям, и т. д. Исследуя эти соотношения, мы пришли в результате к окончательньм формулам (3)-(6).

\section{7. Целочисленная формула и другие обобщения}

В этом пункте мы обсуждаем различные гипотетические обобщения теорем 2.2 и 3.2 . В окончательном виде теория характеристических классов, двойственных циклам мультиособенностей, должна использовать, по-видимому, язык теории пересечений, и вместо когомологий $H^{*}(X)$ комплексного (квазипроективного, возможно, особого) многообразия $X$ следует использовать групшы Чжоу $A_{*} X$ линейных комбинаций алгебраических циклов по модулю рациональной эквивалентности. Заметим, однако, что всюду в этом пункте без большой потери группу $A_{k} X$ можно заменить на групшу $H_{2 k}(X) \simeq H^{2 m-2 k}(X), m=\operatorname{dim} X$, если $X-$ гладко, или на групш $H^{2 n-2 k}(W, W \backslash X), n=\operatorname{dim} W$, если $X$ особо и $W \supset X-$ произвольное гладкое объемлющее многообразие (эти групшы не зависят, с точностью до изоморфизма, от вложения $X \subset W)$. Если $X$ - гладко, то основная конструкция теории пересечений преврашает $A_{*} X$ в кольцо, в котором единищей служит фундаментальный класс $[X]$ многообразия.

Трансверсальность. Пусть задано гладкое собственное отображение $f: X \rightarrow Y$ и $\underline{\alpha}=\left(\alpha_{1}, \ldots, \alpha_{r}\right)$ - класс $\mathscr{K}$-мультиособенностей. В п. 2 мы определили цикл $X(\underline{\alpha}) \subset$ $X^{k}$ для "общего" отображения $f$ как подпространство, являюшееся замыканием множества наборов попарно различных точек $x_{1}, \ldots, x_{r}$ таких, что $f\left(x_{1}\right)=\cdots=f\left(x_{r}\right)$, и таких, что $f$ имеет особенность $\alpha_{i}$ в $x_{i}$. В этом подпункте мы уточняем условие обшности положения и приводим определение схемы мультиособенностей $X(\underline{\alpha})$ для произвольного $f$, не обязательно удовлетворяющего условию общности.

Схема $X(\underline{\alpha})$ определяется задающим ее пучком идеалов. Иньми словами, нам достаточно определить эту схему в окрестности каждой точки $\left(x_{1}, \ldots, x_{r}\right) \in X^{r}$. Мы 
можем предполагать, что среди точек $x_{i}$ имеется $k$ попарно различных $s_{1}, \ldots, s_{k}$ и что $f\left(s_{1}\right)=\cdots=f\left(s_{k}\right)$. Выбрав подходящие координаты в окрестности точек $s_{i}$ и $f\left(x_{i}\right)$, мы можем считать $f$ мультиростком $f:\left(\mathbb{C}^{m}, S\right) \rightarrow\left(\mathbb{C}^{n}, 0\right)$, где $S=\left\{s_{1}, \ldots, s_{r}\right\} \subset \mathbb{C}^{m}$. Рассмотрим деформацию $F$ этого мультиростка вида

$$
F:(x, P) \mapsto f(x)+P(x),
$$

где $P$ - точка пространства $P(m, n, K)$ глобально заданных полиномиальных отображений $\mathbb{C}^{m} \rightarrow \mathbb{C}^{n}$ степени не вьше $K$. Обозначим через $\widetilde{X}^{0}(\underline{\alpha})$ пространство наборов вида $\left(x_{1}, \ldots, x_{r}, P\right) \subset X^{r} \times P(m, n, K)$ таких, что:

1) точки $x_{i}$ попарно различны и каждая из них лежит в некоторой малой окрестности подмножества $S \subset \mathbb{C}^{m}$;

2) $F\left(x_{1}, P\right)=\cdots=F\left(x_{r}, P\right)$;

$3)$ росток отображения $F(\cdot, P)$ в точке $x_{i}$ имеет особенность $\alpha_{i}$.

Из теоремы о конечной определенности следует, что при достаточно больших $K$ ( $K$ определяется мультиособенностью $\underline{\alpha}$ и не зависит от $f)$ подпространство $\widetilde{X}^{0}(\underline{\alpha})$ является гладким (незамкнутьм) подмногообразием ожидаемой размерности. Пусть $\widetilde{X}(\underline{\alpha})$ - его замыкание, рассматриваемое как особое приведенное подмногообразие. Тогда определим $X(\underline{\alpha})$ как схемный прообраз многообразия $\widetilde{X}(\underline{\alpha})$ при естественном вложении $j: X^{r}=X^{r} \times\{0\} \hookrightarrow X^{r} \times P(m, n, K)$.

Нетрудно показать, что схема $X(\underline{\alpha})$ не зависит от выбора $K \gg 0$ и от остального произвола в ее определении, в частности, приведенные ее локальные описания склеиваются в глобально определенную схему.

Заметим, что если мультиросток $f:\left(\mathbb{C}^{m}, S\right) \rightarrow\left(\mathbb{C}^{n}, 0\right)$ конечноопределен, то вместо семейства $F$ можно было бы взять произвольную версальную деформацию мультиростка $f$. Однако приведенная вьше конструкция более общая и не требует конечной определенности особенностей отображения $f$.

Условие трансверсальности отображения $f$ по отношению к мультиособенности $\underline{\alpha}$ заключается в том, что схема $X(\underline{\alpha})$ приведенная, имеет ожидаемую размерность и, кроме того, замкнутое подмногообразие $j^{-1}\left(\widetilde{X}(\underline{\alpha}) \backslash \widetilde{X}^{0}(\underline{\alpha})\right)$ имеет строго меньшую размерность. При вьполнении этого условия определение п. 2, очевидно, эквивалентно приведенному вьше.

Кроме того, построенная схема удовлетворяет следуюшему свойству функториальности: пусть имеется расслоенньй квадрат

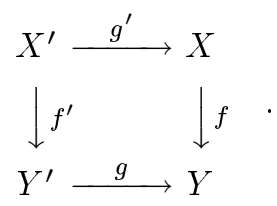

Предположим, что $Y^{\prime}$ гладко и что отображение $f \times g: X \times Y^{\prime} \rightarrow Y^{2}$ трансверсально диагонали $\Delta_{Y} \subset Y^{2}$. Тогда пространство расслоенного произведения $X^{\prime}=$ $X \times_{Y} Y^{\prime}=(f \times g)^{-1} \Delta_{Y}$ также гладко, проекция $f^{\prime}: X^{\prime} \rightarrow Y^{\prime}$ собственна и имеет ту же относительную размерность $\operatorname{dim} Y^{\prime}-\operatorname{dim} X^{\prime}=\operatorname{dim} Y-\operatorname{dim} X$. Мы скажем, 
что отображение $f^{\prime}: X^{\prime} \rightarrow Y^{\prime}$ индуцировано из отображения $f: X \rightarrow Y$ отображением $g$. Кроме того, проекция $g^{\prime}: X \rightarrow X^{\prime}$ индуцирует отображение $g^{\prime r}: X^{\prime r} \rightarrow X^{r}$. Свойство функториальности заключается в том, что схема $X^{\prime}(\underline{\alpha}) \subset X^{\prime r}$, предоставляемая приведенной конструкцией, примененной к отображению $f^{\prime}$, совпадает со схемньм прообразом $\left(g^{\prime r}\right)^{-1} X(\underline{\alpha})$.

Гипотетические обобщения основной теоремы. В дальнейшем нам потребуется следующая лемма. Пусть задан набор гладких отображений $f_{i}: X_{i} \rightarrow Y$, $i=1, \ldots, r$. Положим $\widehat{X}=X_{1} \times_{Y} \cdots \times_{Y} X_{r}=\widehat{f}^{-1} \Delta_{Y}$, где $\widehat{f}=f_{1} \times \cdots \times f_{r}: X_{1} \times$ $\cdots \times X_{r} \rightarrow Y$ и $\Delta_{Y} \simeq Y \subset Y^{r}$ - диагональ. Пусть все отображения $f_{i}$ собственнbl. Тогда проектирование на первьй множитель $p_{1}: \widehat{X} \rightarrow X_{1}$ также собственно, и определен соответствующий гомоморфизм прямого образа

$$
p_{1 *}: A_{*} \widehat{X} \rightarrow A_{*} X_{1}
$$

(Заметим, что проекцию $p_{1}$ можно разложить в композицию $\widehat{X} \hookrightarrow X_{1} \times \cdots \times X_{r} \rightarrow X_{1}$, однако гомоморфизм $p_{1 *}$ раскладьвается в соответствующую композицию гомоморфизмов прямых образов, только если все многообразия $X_{i}$ полны.) Конструкщия произведения-пересечения задает для всякого набора классов $a_{i} \in A_{*} X_{i}$ элемент

$$
a_{1} \times_{Y} \cdots \times_{Y} a_{r}=\Delta_{Y} \cdot\left(a_{1} \times \cdots \times a_{r}\right)=\left(a_{1} \times \cdots \times a_{r}\right) \frown \widehat{f}^{*}\left[\Delta_{Y}\right] \in A_{d} \widehat{X}
$$

где $d=\sum_{i=1} \operatorname{deg} a_{i}-(r-1) \operatorname{dim} Y$.

7.1. ЛЕммА. Если $f_{i}: X_{i} \rightarrow Y, i=1, \ldots, r$, - собственные гладкие отображения, то для всякого набора элементов $a_{i} \in A_{*} X_{i}$ выполняется равенство

$$
p_{1 *} a_{1} \times_{Y} \cdots \times_{Y} a_{r}=a_{1}\left(f_{1}^{*} f_{2 *} a_{2}\right) \cdots\left(f_{1}^{*} f_{r *} a_{r}\right) \in A_{*} X_{1} .
$$

ДоКАЗАТЕЛЬСТВО. В действительности, для корректности определения классов $a_{1} \times_{Y} \cdots \times_{Y} a_{r}$ и справедливости леммы гладкость $X_{i}$ не нужна. Необходимо только, чтобы $Y$ было гладким. Заменив, при необходимости, $X_{i}$ на циклы, представляющие классы $a_{i}$, можно свести лемму к случаю, когда $a_{i}=\left[X_{i}\right]$. Далее, из функториальности конструкции вытекает, что лемму достаточно доказать только для случая $r=2$, т.е. осталось проверить равенство

$$
p_{1 *}\left(\Delta_{Y} \cdot\left(X_{1} \times X_{2}\right)\right)=f_{1}^{*} f_{2 *}\left[X_{2}\right] .
$$

Это равенство доказано, фактически, в [16] (см. доказательство теоремы 9.3).

Вернемся к теории характеристических классов мультиособенностей. Эта теория, в своем окончательном варианте, для гладкого собственного отображения ${ }^{1} f: X \rightarrow Y$ и для всякого набора локальных типов $\mathscr{K}$-особенностей $\underline{\alpha}=\left(\alpha_{1}, \ldots, \alpha_{r}\right)$ должна предоставлять конструкцию класса $\widehat{m}_{\underline{\alpha}} \in A_{d} X(\underline{\alpha})$, где $X(\underline{\alpha})$ - определенная выше схема мультиособенности и $d=\operatorname{dim} X-\operatorname{codim} \underline{\alpha}-$ ее ожидаемая размерность. Этот класс

\footnotetext{
${ }^{1}$ Класс отображений, к которым теория должна быть применима, достаточно широк. Например, при $\ell=\operatorname{dim} Y-\operatorname{dim} X>0$ можно допускать даже отображения, не являющиеся конечными.
} 
должен удовлетворять свойству функториальности по отношению к операции индуцирования отображений, кроме того, если $X(\underline{\alpha})$ имеет ожидаемую размерность, например, в случае выполнения условий трансверсальности, то $\widehat{m}_{\underline{\alpha}}$ должен совпадать с фундаментальньм циклом многообразия $X(\underline{\alpha})$.

Предположим, что конструкция для классов $\widehat{m}_{\underline{\alpha}}$, обладающая перечисленными свойствами, имеется. Заметим, что по построению $X(\underline{\alpha}) \subset X[r]$, где схема $X[r]=X \times_{Y} \cdots \times_{Y} X \subset X^{r}$ определяется как прообраз диагонали при отображении $f^{r}: X^{r} \rightarrow Y^{r}$. Поэтому корректно определены гомоморфизмы прямого образа ограничения на $X(\underline{\alpha})$ отображения $p_{X}: X[r] \rightarrow X$, задаваемого проекцией на первый множитель, и структурного отображения $p_{Y}=f p_{X}: X[r] \rightarrow Y$, и мы можем положить

$$
m_{\underline{\alpha}}=p_{X *} \widehat{m}_{\underline{\alpha}} \in A_{*} X, \quad n_{\underline{\alpha}}=p_{Y *} \widehat{m}_{\underline{\alpha}}=f_{*} m_{\underline{\alpha}} \in A_{*} Y .
$$

В случае выполнения условия трансверсальности для отображения $f$ эти определения классов $m_{\underline{\alpha}}, n_{\underline{\alpha}}$ совпадают с определениями п. 2 .

Пусть $\delta^{r}: X \rightarrow X[r]$ - диагональное вложение. Всякое разбиение $\{1, \ldots, r\}=$ $J_{1} \sqcup \cdots \sqcup J_{k}$ множества индексов $\{1, \ldots, r\}$ в несвязное объединение неупорядоченных непустых подмножеств $J_{1}, \ldots, J_{k}$ мошностей $\left|J_{1}\right|, \ldots,\left|J_{k}\right|$ задает отождествление $X[r]=X\left[\left|J_{1}\right|\right] \times_{Y} \cdots \times_{Y} X\left[\left|J_{k}\right|\right]$ и соответствуюшее этому отождествлению мультидиагональное вложение $\delta^{J_{1}, \ldots, J_{k}}=\delta^{\left|J_{1}\right|} \times_{Y} \cdots \times_{Y} \delta^{\left|J_{r}\right|}: X[k] \rightarrow X[r]$. Это вложение задает для произвольного набора классов $a_{1}, \ldots, a_{k} \in A_{*} X$ их относительное произведение

$$
\left(\delta_{*}^{\left|J_{1}\right|} a_{1}\right) \times_{Y} \cdots \times_{Y}\left(\delta_{*}^{\left|J_{k}\right|} a_{k}\right)=\delta_{*}^{J_{1}, \ldots, J_{k}}\left(a_{1} \times_{Y} \cdots \times_{Y} a_{k}\right) \in A_{*} X[r] .
$$

7.2. ГИПОТЕЗА. Для всякого гладкого собственного отображения $f: X \rightarrow Y$ при надлежащем определении классов $\widehat{m}_{\underline{\alpha}} \in A_{*} X(\underline{\alpha})$ следующая комбинация классов мультиособенностей

$$
\sum_{\{1, \ldots, r\}=J_{1} \sqcup \cdots \sqcup J_{k}}(-1)^{k-1}(k-1) ! \widehat{m}_{\underline{\alpha}_{J_{1}}} \times_{Y} \cdots \times_{Y} \widehat{m}_{\underline{\alpha}_{J_{k}}} \in A_{*} X[r]
$$

представляется ииклом, имеющим носитель на диагонали $\delta^{r} X \subset X[r]$, и выражается в виде $\delta_{*}^{r} R_{\underline{\alpha}}$, где класс $R_{\underline{\alpha}} \in A_{*} X$ задается универсальным многочленом от относительных классов Чженя отображения $f$. Этот многочлен, называемый остаточным многочленом мультиособенности $\underline{\alpha}=\left(\alpha_{1}, \ldots, \alpha_{r}\right)$, зависит только от (неупорядоченного) набора локальных типов $\alpha_{i}$ особенностей ( $и$ числа $\ell=\operatorname{dim} Y-\operatorname{dim} X)$.

При помоши леммы 3.4 из соотношения гипотезы вытекают равенства

$$
\begin{aligned}
\widehat{m}_{\underline{\alpha}} & =\sum_{\{1, \ldots, r\}=J_{1} \sqcup \ldots \sqcup J_{k}} \delta_{*}^{J_{1}, \ldots, J_{k}} R_{\underline{\alpha}_{J_{1}}} \times_{Y} \cdots \times_{Y} R_{\underline{\alpha}_{J_{k}}} \\
& =\delta_{*}^{r} R_{\underline{\alpha}}+\sum_{1 \in J \subsetneq\{1, \ldots, r\}}\left(\delta_{*}^{|J|} R_{\underline{\alpha}_{J}}\right) \times_{Y} \widehat{m}_{\underline{\alpha}}, \quad \bar{J}=\{1, \ldots, r\} \backslash J .
\end{aligned}
$$

Суммирование в этих формулах производится по тем же множествам, что и в формулах (3)-(6). Формулы (3), (5) и (4), (6) получаются из формул гипотезы применением гомоморфизмов $p_{X *}$ и $p_{Y *}$ соответственно с использованием леммы 7.1. 
Утверждение гипотезы и равенства (13), (14) справедливы, по-видимому, при произвольных $\ell=\operatorname{dim} Y-\operatorname{dim} X$, не обязательно положительных. Условие собственности отображения $f$ в указанной формулировке также, по-видимому, не существенно.

Целочисленные формулы. Остаточньй многочлен $R_{\underline{\alpha}}$ гипотезы 7.2 имеет целые коэффициенты, и формулы (13), (14), а следовательно, (3)-(6) справедливы с учетом кручения. Этот факт можно вьвести из гипотезы, поскольку класс в формуле гипотезы целочисленный. Однако имеются более тонкие гипотетические целочисленные равенства, аналогичные формулам [31] для циклов кратных точек. Эксперименты показывают, что в случае, ког да среди особенностей набора $\underline{\alpha}=\left(\alpha_{1}, \ldots, \alpha_{r}\right)$ имеются повторяюшиеся, остаточный многочлен имеет большой числовой множитель.

7.3. ОПРЕДЕЛЕНИЕ. Приведенным остаточным многочленом мультиособенности $\underline{\alpha}=\left(\alpha_{1}, \ldots, \alpha_{r}\right)$ называется многочлен

$$
R_{\underline{\alpha}}^{\mathrm{red}}=R_{\alpha_{1} ; \underline{\alpha}^{\prime}}^{\mathrm{red}}=\frac{1}{\# \operatorname{Aut}\left(\alpha^{\prime}\right)} R_{\underline{\alpha}}
$$

где $\underline{\alpha}^{\prime}=\left(\alpha_{2}, \ldots, \alpha_{r}\right)$. Заметим, что этот многочлен, в отличие от $R_{\underline{\alpha}}$, инвариантен относительно только тех перестановок в наборе $\left(\alpha_{1}, \ldots, \alpha_{r}\right)$, которые сохраняют элемент $\alpha_{1}$. Первый элемент $\alpha_{1}$ набора $\underline{\alpha}=\left(\alpha_{1}, \underline{\alpha}^{\prime}\right)$ мы назовем отмеченныл.м.

Рассмотрим соотношение (5). Левая часть этого равенства является кратным другого целочисленного класса, поскольку $m_{\underline{\alpha}}=\# \operatorname{Aut}\left(\alpha^{\prime}\right) \bar{m}_{\underline{\alpha}}$, где $\bar{m}_{\underline{\alpha}}-$ класс, двойственный соответствующему приведенному циклу мультиособенностей. Правая часть равенства также является кратным, поскольку из симметрии мультииндекса $\underline{\alpha}$ следует, что в правой части имеется много повторяюшихся слагаемых. Разделив обе части (5) на \# $\operatorname{Aut}\left(\alpha_{2}, \ldots, \alpha_{r}\right)$, мы получаем равенство

$$
\bar{m}_{\underline{\alpha}}=R_{\underline{\alpha}}^{\mathrm{red}}+\sum_{\alpha_{1} \in \underline{\alpha}_{J} \subsetneq\left(\alpha_{1}, \ldots, \alpha_{r}\right)} R_{\underline{\alpha}_{J}}^{\mathrm{red}} \bar{n}_{\underline{\alpha}_{J}}
$$

где суммирование производится по всем существенно различным разбиениям набора $\left(\alpha_{1}, \ldots, \alpha_{r}\right)$ на два поднабора, обозначаемые нами через $\underline{\alpha}_{J}$ и $\underline{\alpha}_{\bar{J}}$. При этом мы считаем, что $\underline{\alpha}_{J}$ содержит элемент $\alpha_{1}$ и что этот элемент - отмеченньй. Действительно, каждому слагаемому в равенстве (15) соответствует количество слагаемых равенства (5), равное $\frac{\# \operatorname{Aut}\left(\underline{\alpha}^{\prime}\right)}{\# \operatorname{Aut}\left(\underline{\alpha}_{J}^{\prime}\right) \# \operatorname{Aut}\left(\underline{\alpha}_{J}\right)}$, и каждое из этих слагаемых равно $R_{\underline{\alpha}_{J}} n_{\underline{\alpha}_{J}}=\# \operatorname{Aut}\left(\underline{\alpha}_{J}^{\prime}\right) \# \operatorname{Aut}\left(\underline{\alpha}_{J}\right) \bar{R}_{\underline{\alpha}_{J}} \bar{n}_{\underline{\alpha}}$, откуда и следует равенство (15).

7.4. ГИПОТЕЗА. Приведенные остаточные многочлены имеют иелье коэффициенты, и соотношение (15) выполняется с учетом кручения.

Эта гипотеза тоже имеет обобщение, аналогичное гипотезе 7.2. Оно формулируется в виде соотношений между классами "утонченных" многообразий мультиособенностей, получаемых факторизацией многообразий $X(\underline{\alpha})$ по действию групшы $\mathrm{Aut}(\underline{\alpha})$ автоморфизмов мультииндекса $\underline{\alpha}$ или некоторой ее подгруппы.

Для всех гипотез этого пункта имеется прямое доказательство в частном случае отображений, не имеющих особенностей коранга больше 1, см. [26]. 


\section{8. Изолированные особенности гиперповерхностей и лежандровы характеристические классы}

Почти все результаты о многочленах Тома особенностей и мультиособенностей отображений переносятся на параллельную теорию лежандровых (лагранжевых) особенностей и, как частньй случай, на теорию изолированных (мульти)особенностей гиперповерхностей. Главное отличие состоит в том, что кольцо $\mathscr{C}=H^{*}(B U)$ многочленов от классов Чженя заменяется на кольцо $\mathscr{L}$ универсальных лежандровых характеристических классов. Теория особенностей вещественных лежандровых отображений изложена, например, в [5]. В комплексном случае ситуация аналогична. Напомним кратко определения. Подробности см. в [25].

8.1. ОПРЕДЕЛЕНИЕ. Голоморфное отображение комплексных многообразий $M \rightarrow N$ называется лежандровым, если оно представлено в виде композиции $M \rightarrow P T^{*} N \rightarrow N$, где первое отображение - лежандрова иммерсия, а второе естественная проекция.

В частности, $\operatorname{dim} N-\operatorname{dim} M=1$. Лежандровы отображения образуют специальньй класс отображений, типичные особенности которых отличаются от типичных особенностей общих отображений. Определение лежандровой иммерсии можно найти, например, в [5], [6]. Для приложений часто бьвает достаточно рассматривать лишь частный случай лежандрова отображения, изложенный в следующем примере.

8.2. ПримеР. Рассмотрим гладкое локально тривиальное расслоение и гладкую гиперповерхность в его тотальном пространстве,

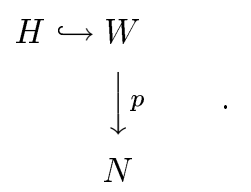

Обозначим через $M \subset H$ подмножество точек, в которых $H$ касается слоев расслоения $p$. Тогда при выполнении условий трансверсальности $M$ является гладким многообразием и его проекция $f$ на базу $N$ расслоения $p$ естественно раскладьвается в композицию $M \rightarrow P T^{*} N \rightarrow N$, в которой первое отображение - лежандрова иммерсия. Эта иммерсия сопоставляет точке $x \in M$ гиперплоскость $p_{*} T_{x} H \subset T_{p(x)} N$, рассматриваемую как точка проективного пространства $P T_{p(x)}^{*} N$.

Точки многообразия области определения лежандрова отображения классифицируются в соответствии с классами изоморфизма изолированных особенностей гиперповерхностей, т.е. классами стабильной $\mathscr{K}$-эквивалентности функций (два ростка функций от, возможно, различного числа переменных назьваются стабильно $\mathscr{K}$-эквивалентными, если они становятся $\mathscr{K}$-эквивалентньми после добавления подходящих невырожденных квадратичных форм от дополнительных переменных). В случае примера 8.2 точке $x \in M \subset H$ сопоставляется особенность гиперповерхности в слое $p^{-1} p(x)$, высекаемой гиперповерхностью $H$. Соответственно, точки многообразия области значения $N$ классифищируются классами изоморфизма мультиособенностей гиперповерхностей. Начало иерархии изолированных особенностей гиперповерхностей приведено на следуюшей диаграмме: 


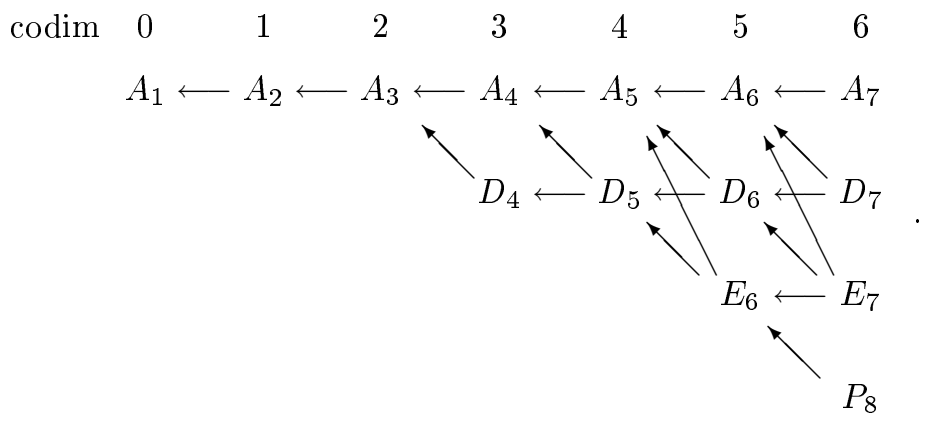

В случае лежандровых отображений кольцо универсальных характеристических классов определяется способом, отличньм от случая обычных отображений.

8.3. ОПРЕДЕЛЕНИЕ [25]. Кольцом универсальных лежсандровых характеристических классов $\mathscr{L}$ назьвается факторкольцо кольца многочленов от образующих $u$, $a_{1}, a_{2}, \ldots$ степеней $\operatorname{deg} u=1, \operatorname{deg} a_{i}=i$ по идеалу соотношений, вытекающих из тождества

$$
\left(1+a_{1}+a_{2}+\cdots\right)\left(1-\frac{a_{1}}{1+u}+\frac{a_{2}}{(1+u)^{2}}-\cdots\right)=1 .
$$

Если положить формально $u=c_{1}(I)$ для некоторого линейного расслоения $I$ и $a_{i}=c_{i}(U)$ для некоторого (виртуального) расслоения $U$ виртуального ранга 0 , то соотношение (16) можно переписать в виде формального равенства

$$
c\left(U^{*} \otimes I+U\right)=1
$$

Кольцо $\mathscr{L}$ не имеет кручения. Аддитивный базис в нем образован мономами вида $u^{k} a_{1}^{i_{1}} a_{2}^{i_{2}} \cdots$, где $i_{j} \in\{0,1\}$.

Для всякого лежандрова многообразия $M \subset P T^{*} N$ имеется канонический гомоморфизм $\mathscr{L} \rightarrow H^{*}(M)$, задаюший на $M$ лежандровы характеристические классы. А именно, нужно положить

$$
u=c_{1}(I), \quad a_{i}=c_{i}\left(f^{*} T N-T M-I\right),
$$

где линейное расслоение $I$ - нормальное расслоение контактной структуры (оно совпадает также с расслоением, сопряженньм тавтологическому линейному на $\left.P T^{*} N\right)$ и $f: M \rightarrow N$ - ограничение на $M$ естественной проекции $P T^{*} N \rightarrow N$.

В случае отображения примера 8.2 лежандровы характеристические классы, соответствующие построенной лежандровой иммерсии, равны

$$
u=[H]=c_{1}(I), \quad a_{i}=c_{i}\left(V^{*} \otimes I-V\right)
$$

где линейное расслоение $I$ определено на всем $W$ как расслоение дивизора $H$ (его ограничение на $H$ совпадает с нормальным расслоением гиперповерхности $H$ ) и $V=\operatorname{ker} p_{*}-$ подрасслоение в касательном расслоении к $W$, состояшее из векторов, 
касающихся слоев расслоения $p$. Соотношение (16) в этом случае вытекает из несложной выкладки

$$
\left(V^{*} \otimes I-V\right)^{*} \otimes I+\left(V^{*} \otimes I-V\right)=0 .
$$

В обшем случае соотношение (16) вытекает из следуюших соображений. Касательное расслоение на $P T^{*} N$ имеет естественное подрасслоение $E$ коразмерности 1 , называемое канонической контактной структурой. Слой этого расслоения над точкой $y \in P T_{x}^{*} N$ определяется равенством $E_{y}=\pi^{-1} \widehat{y}$, где $\widehat{y} \subset T_{x} N$ - отвечающая точке $y$ касательная гиперплоскость и где отображение $\pi: T_{y}\left(P T^{*} N\right) \rightarrow T_{x} N$ индуцировано естественной проекцией. Обозначим через $I=T\left(P T^{*} N\right) / E$ соответствуюшее линейное факторрасслоение. Коммутатор векторных полей задает кососимметрическое билинейное спаривание $E \times E \rightarrow I$. Это спаривание невырожденно (это равносильно невырожденности контактной структуры). Кроме того, плоскость $T_{y} M \subset E_{y}$, касательная к лежандрову подмногообразию, является лагранжевым подпространством (т.е. изотропным половинной размерности) относительно этого спаривания. Из невырожденности спаривания вытекает изоморфизм $E / T M \simeq T^{*} M \otimes I$. С другой стороны, расслоение $E$ выражается в терминах многообразия $N$ при помоши точных последовательностей

$$
0 \rightarrow E \rightarrow T\left(P T^{*} N\right) \rightarrow I \rightarrow 0, \quad 0 \rightarrow \mathbb{C} \rightarrow T^{*} N \otimes I \rightarrow T\left(P T^{*} N\right)-T N \rightarrow 0 .
$$

Собирая вместе полученные соотношения, мы получаем, что в групе $K(M)$ виртуальных векторных расслоений на $M$ выполняется равенство

$$
E=T M+T^{*} M \otimes I=T N+T^{*} N \otimes I-\mathbb{C}-I,
$$

т.е.

$$
(T N-T M-I)^{*} \otimes I+(T N-T M-I)=0,
$$

что и утверждает тождество (16).

В действительности, аналогично случаю обычных отображений и их особенностей, имеется конструкция классифищирующего пространства лежандровых особенностей [25]. Это пространство гомотопически эквивалентно стабильному лежандрову грассманиану, и его кольцо когомологий изоморфно $\mathscr{L}$. Точки классифищируюшего пространства разбиты на страты, отвечающие различным типам лежандровых особенностей (т.е. различным типам изолированных особенностей гиперповерхностей). Для всякого лежандрова многообразия $M \subset P T^{*} N$ имеется конструкция классифицируюшего отображения многообразия $M$ в классифицируюшее пространство, которое индуцирует как разбиение на различные типы особенностей, так и характеристические классы. Классы $u, a_{i}$ на лежандровом многообразии - это обратные образы стандартных образуюших когомологий классифицируюшего пространства.

Применение гомоморфизма Гизина $f_{*}: H^{*}(M) \rightarrow H^{*}(N)$ позволяет переносить лежандровы характеристические классы с лежандрова многообразия $M$ на $N$. Аналогично случаю обычных отображений, возникает коммутативная диаграмма

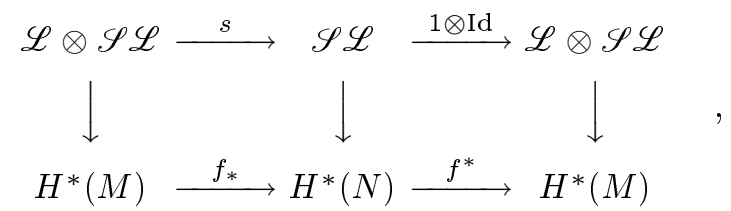


где $\mathscr{S} \mathscr{L}$ - полиномиальное кольцо

$$
\mathscr{S} \mathscr{L}=\operatorname{Sym} \mathscr{L}=1 \oplus \mathscr{L} \oplus \operatorname{Sym}^{2} \mathscr{L} \oplus \cdots
$$

(без дополнительных соотношений).

(Мульти)особенности лежандровых отображений, равно как и изолированные (мульти)особенности гиперповерхностей, нумеруются наборами вида $\underline{\alpha}=$ $\left(\alpha_{1}, \ldots, \alpha_{r}\right)$, где $\alpha_{i}-$ класс стабильной $\mathscr{K}$-эквивалентности функций. Аналогично случаю обычных отображений, всякий тип $\underline{\alpha}$ мультиособенности задает соответствуюшие циклы $M(\underline{\alpha}) \subset M^{r}, p_{M}(M(\underline{\alpha})) \subset M, p_{N}(M(\underline{\alpha})) \subset N$ и двойственные им классы

$$
\bar{m}_{\underline{\alpha}}=\left[p_{M}(M(\underline{\alpha}))\right] \in H^{*}(M), \quad \bar{n}_{\underline{\alpha}}=\left[p_{N}(M(\underline{\alpha}))\right] \in H^{*}(N),
$$

где многообразия $p_{M}(M(\underline{\alpha})), p_{N}(M(\underline{\alpha}))$ рассматриваются с приведенной структурой, а также кратные им классы

$$
m_{\underline{\alpha}}=\# \operatorname{Aut}\left(\underline{\alpha}^{\prime}\right) \bar{m}_{\underline{\alpha}}, \quad n_{\underline{\alpha}}=\# \operatorname{Aut}(\underline{\alpha}) \bar{n}_{\underline{\alpha}}
$$

где $\operatorname{Aut}(\underline{\alpha})$ - группа автоморфизмов мультииндекса $\underline{\alpha}=\left(\alpha_{1}, \ldots, \alpha_{r}\right)$ и $\operatorname{Aut}\left(\underline{\alpha}^{\prime}\right)=$ $\operatorname{Aut}\left(\alpha_{2}, \ldots, \alpha_{r}\right)$ - подгруппа в $\operatorname{Aut}(\underline{\alpha})$, содержашая только те перестановки, которые оставляют на месте первый элемент.

8.4. ТЕОРемА. Для всякого набора классов изолированных особенностей гиперповерхностей $\underline{\alpha}=\left(\alpha_{1}, \ldots, \alpha_{r}\right)$ существует универсальный остаточный лежандров характеристический класс $R_{\underline{\alpha}} \in \mathscr{L}$ такой, что классы $m_{\underline{\alpha}}, n_{\underline{\alpha}}$ лежсандровых мультиособенностей лежсандрова отображения $f: M \rightarrow \overline{P T}^{*} \bar{N} \rightarrow N$ общего положения задаются формулами, аналогичными формулам теоремы 3.2 ,

$$
\begin{aligned}
& m_{\underline{\alpha}}=\sum_{J_{1} \sqcup \cdots \sqcup J_{k}=\{1, \ldots, r\}} R_{\underline{\alpha}_{J_{1}}} S_{\underline{\alpha}_{J_{2}}} \cdots S_{\underline{\alpha}_{J_{k}}}, \\
& n_{\underline{\alpha}}=s\left(m_{\underline{\alpha}}\right)=\sum_{J_{1} \sqcup \cdots \sqcup J_{k}=\{1, \ldots, r\}} S_{\underline{\alpha}_{J_{1}}} \cdots S_{\underline{\alpha}_{J_{k}}},
\end{aligned}
$$

где $S_{\underline{\alpha}}=s\left(R_{\underline{\alpha}}\right)$ и суммирование производится по всевозможсным разбиениям мноэествв $\{1, \ldots, r\}$ в несвязное облединение непустых неупорядоченных подмножеств $\{1, \ldots, r\}=J_{1} \sqcup \cdots \sqcup J_{k}, k \geqslant 1$. Подмножсество, содерхащее әлемент $1 \in\{1, \ldots, r\}$, обозначается через $J_{1}$.

Кроме того, остаточный класс $R_{\underline{\alpha}}$ не зависит от порядка локальных особенностей $\alpha_{i}$, входящих в набор $\underline{\alpha}=\left(\alpha_{1}, \ldots, \alpha_{r}\right)$.

Топологическое обоснование этой теоремы проводится по той же схеме, что и аналогичные рассуждения п. 6, использовавшиеся при обосновании соответствуюшего результата для обычных отображений. Основное отличие состоит в том, что пространство Тома $M U(n)$ классифицирующего векторного расслоения над $B U(n)$ нужно заменить на пространство Тома тавтологического векторного расслоения над соответствующим лагранжевым (или лежандровым) грассманианом. Мы не приводим подробностей этих рассуждений, поскольку, как и в случае теорем $2.2,3.2$, мы надеемся получить доказательство, основанное на методах теории пересечений. 
8.5. ПримеР. Аналогично случаю обычных отображений, мы получаем, в частности, следующую формулу для кратных точек лежсандровых отображсний:

$$
\bar{m}_{r}=f^{*}\left(\bar{n}_{r-1}\right)-\sum_{i=1}^{r-1} p_{r-i} \bar{m}_{r}
$$

где $p_{i} \in \mathscr{L}$ - универсальные лежандровы характеристические классы, определенные соотношением

$$
\begin{gathered}
1+p_{1} t+p_{2} t^{2}+\cdots=\left(1+\frac{1}{1 !} R_{A_{1}^{2}} t+\frac{1}{2 !} R_{A_{1}^{3}} t^{2}+\cdots\right)^{-1}, \\
p_{1}=u+3 a_{1}, \quad p_{2}=-4\left(u a_{1}+3 a_{2}\right), \quad p_{3}=16 u^{2} a_{1}+59 u a_{2}+45 a_{1} a_{2}+78 a_{3}, \ldots
\end{gathered}
$$

Большое количество других примеров будет приведено в последующих пунктах.

\section{9. Вычисление лежандровых остаточных классов}

Для классов моноособенностей вплоть до коразмерности 6 лежандровы характеристические классы вычислены в [25]. Эти вычисления проведены двумя независимыми способами, при помоши классических методов разрешения, использования гомоморфизма Гизина и т. д., а также при помощи метода Римани использования симметрий. Метод Римани менее геометричен и полагается на теорему об априорном сушествовании соответствуюших многочленов. Однако этот метод требует меньшего объема вычислений и может быть с успехом приспособлен к вычислению классов мультиособенностей. Для особенностей критических точек вплоть до коразмерности 6 необходимая информация для вычисления остаточных классов этим методом приведена ниже. Все особенности этой таблищы квазиоднородны, поэтому группа симметрий каждой из них - конечное расширение группы $U(1)$, и $H^{*}\left(B G_{\alpha}, \mathbb{Q}\right) \simeq \mathbb{Q}[t]$. Особенность $P_{8}$ образует однопараметрическое семейство неэквивалентных функций, зависящих от параметра $a$. Индивидуальные члены этого семейтсва имеют коразмерность 7, но весь класс целиком имеет коразмерность 6.

\begin{tabular}{|c|c|c|c|c|}
\hline$\alpha$ & Нормальная форма & $\operatorname{codim}$ & $u$ & $a$ \\
\hline$A_{k}$ & $x^{k+1}$ & $k-1$ & $(k+1) t$ & $\frac{1+k t}{1+t}$ \\
\hline$D_{k}$ & $y^{k-1}+x^{2} y$ & $k-1$ & $(2 k-2) t$ & $\frac{(1+(2 k-4) t)(1+k t)}{(1+2 t)(1+(k-2) t)}$ \\
\hline$E_{6}$ & $x^{3}+y^{4}$ & 5 & $12 t$ & $\frac{(1+9 t)(1+8 t)}{(1+3 t)(1+4 t)}$ \\
\hline$E_{7}$ & $x^{3}+x y^{3}$ & 6 & $9 t$ & $\frac{(1+7 t)(1+6 t)}{(1+2 t)(1+3 t)}$ \\
\hline$P_{8}$ & $x^{3}+y^{3}+z^{3}+a x y z$ & 6 & $3 t$ & $\frac{(1+2 t)^{3}}{(1+t)^{3}}$ \\
\hline
\end{tabular}


Список вычисленных остаточных классов имеется в [28]. Для мультиособенностей вплоть до коразмерности 4 эти классы приведены в следующей таблище.

\section{Остаточные классы $R_{\underline{\alpha}}$ коразмерности не выше 4 лежандровых отображений}

$$
\begin{aligned}
R_{A_{1}} & =1 \\
R_{A_{2}} & =a_{1} \\
R_{A_{1}^{2}} & =-u-3 a_{1} \\
R_{A_{3}} & =u a_{1}+3 a_{2} \\
R_{A_{1} A_{2}} & =-6\left(u a_{1}+2 a_{2}\right) \\
R_{A_{1}^{3}} & =2\left(u^{2}+19 u a_{1}+30 a_{2}\right) \\
R_{A_{4}} & =u^{2} a_{1}+4 u a_{2}+3 a_{1} a_{2}+6 a_{3} \\
R_{D_{4}} & =-u a_{2}+a_{1} a_{2}-2 a_{3} \\
R_{A_{1} A_{3}} & =-4\left(2 u^{2} a_{1}+5 u a_{2}+6 a_{1} a_{2}+3 a_{3}\right) \\
R_{A_{2}^{2}} & =-3\left(3 u^{2} a_{1}+8 u a_{2}+7 a_{1} a_{2}+6 a_{3}\right) \\
R_{A_{1}^{2} A_{2}} & =24\left(3 u^{2} a_{1}+7 u a_{2}+6 a_{1} a_{2}+3 a_{3}\right) \\
R_{A_{1}^{4}} & =-6\left(u^{3}+111 u^{2} a_{1}+239 u a_{2}+171 a_{1} a_{2}+78 a_{3}\right) \\
R_{A_{5}} & =u^{3} a_{1}-4 u^{2} a_{2}+16 u a_{1} a_{2}-12 u a_{3}+27 a_{1} a_{3}+6 a_{4} \\
R_{D_{5}} & =-2\left(2 u^{2} a_{2}-2 u a_{1} a_{2}+7 u a_{3}-3 a_{1} a_{3}+6 a_{4}\right) \\
R_{A_{1} A_{4}} & =-10\left(u^{3} a_{1}-4 u^{2} a_{2}+14 u a_{1} a_{2}-16 u a_{3}+21 a_{1} a_{3}-6 a_{4}\right) \\
R_{A_{1} D_{4}} & =4\left(5 u^{2} a_{2}-5 u a_{1} a_{2}+16 u a_{3}-6 a_{1} a_{3}+12 a_{4}\right) \\
R_{A_{2} A_{3}} & =-6\left(2 u^{3} a_{1}-10 u^{2} a_{2}+28 u a_{1} a_{2}-39 u a_{3}+39 a_{1} a_{3}-18 a_{4}\right) \\
R_{A_{1}^{2} A_{3}} & =2\left(56 u^{3} a_{1}-220 u^{2} a_{2}+684 u a_{1} a_{2}-951 u a_{3}+891 a_{1} a_{3}-522 a_{4}\right) \\
R_{A_{1} A_{2}^{2}} & =18\left(7 u^{3} a_{1}-20 u^{2} a_{2}+74 u a_{1} a_{2}-96 u a_{3}+95 a_{1} a_{3}-50 a_{4}\right) \\
R_{A_{1}^{3} A_{2}} & =-48\left(28 u^{3} a_{1}-55 u^{2} a_{2}+250 u a_{1} a_{2}-318 u a_{3}+300 a_{1} a_{3}-180 a_{4}\right) \\
R_{A_{1}^{5}} & =24\left(u^{4}+671 u^{3} a_{1}-701 u^{2} a_{2}+4863 u a_{1} a_{2}-5844 u a_{3}+5490 a_{1} a_{3}-3420 a_{4}\right) \\
& =-10
\end{aligned}
$$

\section{0. Приложения к исчислительной геометрии}

Необъятное количество (несколько сотен) конкретных исчислительных результатов, вытекающих из формул для классов мультиособенностей, невозможно привести здесь полностью ввиду огромного количества занимаемого ими места (см. страницу [28] в интернете). Здесь мы приведем только несколько примеров.

Исчисление особых кривых на поверхностях. Простейшим приложением описанной вьше теории является исчисление особых кривых на поверхностях. Рассмотрим $n$-мерную линейную систему $|L|$ дивизоров на компактной неособой комплексной поверхности $S$. Тогда формула теоремы 8.4 предсказывает число особых кривых этой системы, имеющих предписанньй набор $\underline{\alpha}$ особенностей и проходящих через 
общие $n-\operatorname{codim} \underline{\alpha}$ точек. Пространством параметров $N=\mathbb{C} P^{n}$ в этой задаче служит пространство параметров системы. Расслоение $W \rightarrow N-$ тривиальное расслоение $N \times S \rightarrow N$, гиперповерхность $H \subset N \times S$ - многообразие инцидентности, состоящее из пар вида (кривая семейства, точка на этой кривой); $M \subset H$-многообразие всех особых точек всех кривых семейства.

Лежандровы характеристические классы, отвечающие за эту ситуацию, следуюшие. Пусть $h \in H^{2}(S)$ - первьй класс Чженя системы, $1+c_{1}+c_{2}=c\left(T^{*} S\right)$, и пусть $t$ - класс гиперплоскости в $N=\mathbb{C} P^{n}$. Тогда

$$
u=c_{1}(I)=[H]=t+h, \quad a=c\left(T^{*} S \otimes I-T S\right)=\frac{(1+t+h)^{2}+(1+t+h) c_{1}+c_{2}}{1-c_{1}+c_{2}},
$$

иньми словами,

$$
\begin{gathered}
a_{1}=2\left(h+t+c_{1}\right), \quad a_{2}=\left(h+t+c_{1}\right)\left(h+t+2 c_{1}\right), \quad a_{3}=t\left(2 h c_{1}+t c_{1}+3 c_{1}^{2}-2 c_{2}\right), \\
a_{4}=t^{2}\left(c_{1}^{2}-c_{2}\right), \quad a_{k}=0 \text { при } k \geqslant 5 .
\end{gathered}
$$

Гомоморфизм $f_{*}: H^{*}(M) \rightarrow H^{*}(N)$ можно разложить в композицию $f_{*}=p_{*} i_{*}$, где $i: M \rightarrow N \times S$ - вложение и $p: N \times S \rightarrow N$ - проекция на первьй множитель. Тогда $i_{*}$ задается умножением на класс подмногообразия $M$ в $S \times N$,

$$
\begin{aligned}
{[M] } & =i_{*}(1)=c_{2}\left(T^{*} S \otimes I\right) \cdot[H]=\left((t+h)^{2}+c_{1}(t+h)+c_{2}\right)(t+h) \\
& =t^{3}+t^{2}\left(3 h+c_{1}\right)+t\left(3 h^{2}+2 h c_{1}+c_{2}\right),
\end{aligned}
$$

а гомоморфизм $p_{*}$ определяется следуюшим образом. Он коммутирует с умножением на $t$, на мономах степени 2 от классов $h, c_{1}, c_{2}$ он равен соответствующему характеристическому числу,

$$
\begin{array}{ll}
d:=p_{*}\left(h^{2}\right)=L \cdot L, & k:=p_{*}\left(h c_{1}\right)=L \cdot K_{S}, \\
s:=p_{*}\left(c_{1}^{2}\right)=K_{S} \cdot K_{S}, & x:=p_{*}\left(c_{2}\right)=c_{2} \frown[S]
\end{array}
$$

на остальных мономах от классов $h, c_{1}, c_{2}$ гомоморфизм $p_{*}$ обрашается в нуль. В качестве следствия мы получаем, что для произвольной мультиособенности $\underline{\alpha}$ класс $S_{\underline{\alpha}}=f_{*} R_{\underline{\alpha}}$ является универсальной линейной комбинацией чисел $d, k, s, x$, умноженной на $t^{\overline{\operatorname{co}} \operatorname{dim}} \underline{\alpha}$, и равенства теоремы 8.4 сводятся к следующему результату, частными случаями которого являются гипотеза Гёттше [18] инекоторые из известных формул Клеймана и Пино [34], [35].

10.1. ТЕОРемА. Для всякого типа мультиособенностей плоских кривьх $\underline{\alpha}=$ $\left(\alpha_{1}, \ldots, \alpha_{r}\right)$ существует универсальная челочисленная линейная комбинация $S_{\underline{\alpha}}$ чисел $d, k, s, x$ такая, что для всякой поверхности и для всякой достаточно общей линейной системы L на ней число особых кривых системы, имеющих заданный набор $\underline{\alpha}$ особенностей и проходящих через заданные $n-\operatorname{codim} \underline{\alpha}$ точек общего положения, дается формулами теоремы 8.4:

$$
N_{\underline{\alpha}}=\frac{1}{\# \operatorname{Aut}(\underline{\alpha})} \sum_{J_{1} \sqcup \ldots \sqcup J_{l}=\{1, \ldots, r\}} S_{\underline{\alpha}_{J_{1}}} \cdots S_{\underline{\alpha}_{J_{l}}} .
$$


Условие общности системы формулируется следующим образом. Обозначим через $N_{\text {reg }} \subset N$ подмножество тех кривых $C$ системы, которые имеют конечное число особых точек и удовлетворяют следующему условию трансверсальности: деформация кривой $C$, индуцированная системой, является версальной деформацией для мультиростка особенностей кривой $C$. Обозначим через $\Sigma=N \backslash N_{\text {reg }}$ его дополнение. Тогда формулы теоремы справедливы для всех классов $\underline{\alpha}$ коразмерности

$$
\operatorname{codim} \underline{\alpha}<\operatorname{codim} \Sigma \text {. }
$$

10.2. ПРИмеР. Обозначим через $N_{i}=N_{A_{1}, \ldots, A_{1}}$ степень соответствующего многообразия нодальных кривых, имеющих $i$ простых двойных точек. Составим из этих чисел формальньй ряд $T(t)=\sum_{i} N_{i} t^{i}$. Тогда, по формулам п. 4, эта производящая функция имеет вид

$$
T(t)=\exp \left(V_{1}(t) d+V_{2}(t) k+V_{3}(t) s+V_{4}(t) x\right)
$$

где коэффициент при $t^{i}$ в ряду, представленном показателем экспоненты, равен

$$
\frac{1}{i !} S_{A_{1}, \ldots, A_{1}}
$$

( $i$ вхождений особенности $A_{1}$ простого двойного пересечения). Приведенная формула является одной из формулировок гипотезы Гёттше [18] (в этой работе ряды $V_{i}(t)$ обозначаются через $A_{i}$ ). Необходимо отметить, что хотя аргументы п. 6 дают топологическое обоснование теоремы 10.1, а вместе с ней и гипотезы Гёттше, все же остается открытым вопрос о получении доказательства методами алгебраической геометрии.

Для мультиособенностей малых коразмерностей числа $S_{\underline{\alpha}}$ приведены ниже. Эти выражения совпадают с соответствующими выражениями, вычисленньми Клейманом и Пино [34], [35] для нодальных мультиособенностей $A_{1}^{r}$ (полный список этих чисел для всех мультиособенностей вплоть до коразмерности 8 приведен в [28]).

$$
\begin{gathered}
\quad \text { codim }=1: \\
S_{A_{1}}=3 d+2 k+x \\
\quad \operatorname{codim}=2: \\
S_{A_{2}}=12 d+12 k+2 s+2 x \\
S_{A_{1}^{2}}=-42 d-39 k-6 s-7 x \\
\quad \operatorname{codim}=3: \\
S_{A_{3}}=50 d+64 k+17 s+5 x \\
S_{A_{1} A_{2}}=-240 d-288 k-72 s-24 x \\
S_{A_{1}^{3}}=1380 d+1576 k+376 s+138 x \\
\quad \operatorname{codim}=4: \\
S_{A_{4}}=180 d+280 k+100 s \\
S_{D_{4}}=15 d+20 k+5 s+5 x
\end{gathered}
$$




$$
\begin{aligned}
S_{A_{1} A_{3}}= & -1260 d-1820 k-596 s-60 x \\
S_{A_{2}^{2}}= & -1260 d-1800 k-588 s-48 x \\
S_{A_{1}^{2} A_{2}} & =9000 d+12360 k+3864 s+456 x \\
S_{A_{1}^{4}}= & -72360 d-95670 k-28842 s-3888 x \\
& \quad \operatorname{dim}=5: \\
S_{A_{5}}= & 630 d+1140 k+498 s-60 x \\
S_{D_{5}}= & 84 d+132 k+44 s+20 x \\
S_{A_{1} A_{4}}= & -5460 d-9240 k-3740 s+200 x \\
S_{A_{1} D_{4}}= & -420 d-624 k-196 s-100 x \\
S_{A_{2} A_{3}}= & -6300 d-10332 k-4044 s+60 x \\
S_{A_{1}^{2} A_{3}}= & 52920 d+84180 k+31816 s+240 x \\
S_{A_{1} A_{2}^{2}}= & 53676 d+84456 k+31716 s+72 x \\
S_{A_{1}^{3} A_{2}}= & -505008 d-770112 k-279792 s-5616 x \\
S_{A_{1}^{5}}= & 5225472 d+7725168 k+2723400 s+84384 x \\
& \cdots \\
S_{A_{1}^{6}}= & -481239360 d-778065120 k-308078520 s+7918560 x \\
S_{A_{1}^{7}}= & 53917151040 d+93895251840 k+40747613760 s-2465471520 x
\end{aligned}
$$

Например, в частном случае полной линейной системы плоских кривых степени $m$ мы имеем $S=\mathbb{C} P^{2}, n=\frac{(m+1)(m+2)}{2}-1, d=m^{2}, k=3 m, s=9, x=3$. Формула теоремы справедлива, предположительно, при

$$
\operatorname{codim} \underline{\alpha} \leqslant 2(m-1)
$$

(аналогичная оценка сформулирована в виде гипотезы в [18]). Ограничение codim $\Sigma \leqslant$ $2 m-1$ вытекает из того, что $\Sigma$ содержит подмногообразие коразмерности $2 m-1$, состоящее из кривых, которые распадаются на кривую степени $m-2$ и двойную прямую. Такие кривые имеют неизолированные особенности, т.е. особенности, которые бесконечно вырождены с точки зрения теории особенностей.

10.3. ПримеР. Для плоских проективных кривых степени $m=3$ приведенные формулы справедливы при $\operatorname{codim} \underline{\alpha} \leqslant 4$, и мы получаем:

\begin{tabular}{|c||c|c|c|c|c|c|c|c|c|c|c|c|}
\hline$\underline{\alpha}$ & $A_{1}$ & $A_{2}$ & $A_{1}^{2}$ & $A_{3}$ & $A_{1} A_{2}$ & $A_{1}^{3}$ & $A_{4}$ & $D_{4}$ & $A_{1} A_{3}$ & $A_{2}^{2}$ & $A_{1}^{2} A_{2}$ & $A_{1}^{4}$ \\
\hline$N_{\underline{\alpha}}$ & 12 & 24 & 21 & 42 & 0 & 15 & 0 & 15 & 0 & 0 & 0 & 0 \\
\hline
\end{tabular}

Все эти числа легко проверить элементарными прямыми вычислениями. Например, $N_{A_{1}}=12$ - число рациональных кривых степени 3 , проходяших через обшие 8 точек, $N_{D_{4}}=15$ - число конфигураций из трех прямых, пересекающихся в одной точке и проходящих через заданные 5 точек плоскости и т. д. 
10.4. ПримеР. Количество плоских кривых степени 4 с 3 двойными точками, проходяших через обшие 11 точек плоскости, равно $\left.N_{A_{1}^{3}}\right|_{m=4}=675$. Это число включает $\left(\begin{array}{c}11 \\ 2\end{array}\right)=55$ приводимых кривых, распадаюшихся на прямую и кубику, и 620 рациональных кривых.

10.5. ПримеР. Подставляя $m=5$ в формулу для $N_{A_{1}^{6}}$, мы получаем число 109781 кривых степени 5 с 6 двойньми точками, проходящих через 14 заданных точек плоскости. Для каждой такой кривой $C$ мы имеем одну из трех следуюших возможностей: а) кривая $C$ распадается на кубику и квадрику; б) кривая $C$ распадается на прямую и кривую степени 4 с двумя двойньми точками; в) $C$-неприводимая рациональная кривая. Число кривых типа а) равно $\left(\begin{array}{c}14 \\ 5\end{array}\right)=2002$. Число кривых типа б) равно $225\left(\begin{array}{c}14 \\ 2\end{array}\right)=20475$ (число $225=\left.\operatorname{deg} A_{1}^{2}\right|_{m=4}$ кривых степени 4 с двумя двойными точками, проходяших через заданные 12 точек плоскости, берется из той же таблицы). Следовательно, число рациональных кривых типа в) равно 109781 - 2002 $20475=87304$, что согласуется с известными вычислениями из [36].

Более общим образом, рассмотрим число $N_{m, g}$ неприводимых плоских кривых степени $m$ и рода $g$, проходящих через заданные общие $3 m-1-g$ точек. Эти числа изучаются в теории инвариантов Громова-Виттена. Они связаны с числами $M_{m, k}$ всех плоских кривых степени $m$ с $k$ двойньми точками, проходяших через общие $\frac{m(m+3)}{2}-k$ точек, при помощи следующего соотношения, которое обобщает вычисления, проведенные выше для $m \leqslant 5$ :

$$
1+\sum_{m, k} M_{m, k} \frac{x^{m} y^{\frac{m(m+3)}{2}-k}}{\left(\frac{m(m+3)}{2}-k\right) !}=\exp \left(\sum_{m, g} N_{m, g} \frac{x^{m} y^{3 m+g-1}}{(3 m+g-1) !}\right) .
$$

$\mathrm{K}$ сожалению, наш метод для вычисления чисел $M_{m, k}$, а следовательно, чисел $N_{m, g}$, дает правильный ответ только при $k \leqslant 2(m-1)$. При больших же $k$ нужно учитьвать вклад неизолированных особенностей.

Исчисление касаний проективных многообразий с гиперплоскостями. Рассмотренная вьше задача исчисления плоских особых кривых является частньм случаем следующей более общей задачи. Пусть на неособом многообразии $V$ задана $n$-мерная линейная система дивизоров. Параметры этой системы образуют проективное пространство $N \simeq \mathbb{C} P^{n}$. Требуется определить степень подмногообразия параметров, для которых дивизор системы имеет предписанный набор особенностей. Для простоты будем считать, что эта система задает проективное вложение. Тогда в качестве дивизоров системы выступают гиперплоские сечения. (Например, в случае задачи исчисления плоских кривых степени $m$ подмногообразием $V$ служит вложение Веронезе $\mathbb{C} P^{2} \rightarrow \mathbb{C} P^{\frac{(d+1)(d+2)}{2}-1}$.) Легко видеть, что мы находимся в ситуации примера 8.2 , где $N=\mathbb{C} P^{n *}$ - многообразие гиперплоскостей в проективном пространстве $\mathbb{C} P^{n}, W=V \times N, p: V \times N \rightarrow N$ - тривиальное расслоение и $H \subset V \times N$ - гиперповерхность инцидентности, образованная парами вида (точка $x \in V$; проходящая через нее гиперплоскость в $\left.\mathbb{C} P^{n}\right)$. Лежандровьм многообразием $M$ соответствующего лежандрова отображения является пространство проективизированного конормального расслоения на $V$, и лежандровы характеристические классы легко вычисляются при помоши подходяших формул присоединения. 
Пусть, например, $V \subset \mathbb{C} P^{n}$ - гиперповерхность степени $d$. Тогда $M=V$. Конормальное расслоение к $V$ является $d$-й тензорной степенью линейного тавтологического расслоения на $\mathbb{C} P^{n}$, откуда

$$
u=c_{1}(I)=c_{1}\left(\nu_{V}\right)=[V]=d t,
$$

где $t=c_{1}(\mathscr{O}(1))$ - класс гиперплоского сечения. Далее, из точных последовательностей

$$
0 \rightarrow T V \rightarrow T_{V} \mathbb{C} P^{n} \rightarrow \nu_{V} \rightarrow 0, \quad 0 \rightarrow \mathbb{C} \rightarrow \mathscr{O}^{n+1}(1) \rightarrow T \mathbb{C} P^{n} \rightarrow 0
$$

мы получаем $c(T V)=c\left(\mathscr{O}^{n+1}(1)-\nu_{V}-\mathbb{C}\right)=(1+t)^{n+1} /(1+d t)$ и

$$
a=c\left(T^{*} V \otimes I-T V\right)=\left(\frac{1+(d-1) t}{1+t}\right)^{n+1} .
$$

Числа особых гиперплоских сечений различных типов гиперповерхности $V$ степени $d$ в $\mathbb{C} P^{n}$ для малых $n$ приведены ниже. ${ }^{2}$

\section{Мультикасательные прямые к кривой в $\mathbb{C} P^{2}$}

$$
\begin{aligned}
& \# A_{2}=3 d(d-2) \\
& \# A_{1}^{2}=\frac{1}{2} d(d-3)(d-2)(d+3)
\end{aligned}
$$

Мультикасательные плоскости к поверхности в $\mathbb{C} P^{3}$

$$
\begin{aligned}
\# A_{3} & =2 d(11 d-24)(d-2) \\
\# A_{1} A_{2} & =4 d(d-3)(d-2)\left(d^{3}+3 d-16\right) \\
\# A_{1}^{3} & =\frac{1}{6} d(d-2)\left(d^{7}-4 d^{6}+7 d^{5}-45 d^{4}+114 d^{3}-111 d^{2}+548 d-960\right)
\end{aligned}
$$

\section{Мультикасательные гиперплоскости к гиперповерхности в $\mathbb{C} P^{4}$}

$$
\begin{aligned}
\# A_{4}= & 5 d(17 d-36)(3 d-7)(d-2) \\
\# D_{4}= & 20 d(d-2)^{3} \\
\# A_{1} A_{3}= & 5 d(d-2)\left(7 d^{6}-43 d^{5}+102 d^{4}-118 d^{3}-245 d^{2}+1309 d-1404\right) \\
\# A_{2}^{2}= & \frac{5}{2} d(d-3)(d-2)\left(5 d^{5}-15 d^{4}+25 d^{3}-5 d^{2}-273 d+452\right) \\
\# A_{1}^{2} A_{2}= & \frac{5}{2} d(d-2)\left(d^{10}-8 d^{9}+28 d^{8}-56 d^{7}-6 d^{6}+398 d^{5}-1028 d^{4}\right. \\
& \left.+1196 d^{3}+1317 d^{2}-8034 d+8424\right) \\
\# A_{1}^{4}= & \frac{1}{24} d(d-2)\left(d^{14}-10 d^{13}+46 d^{12}-128 d^{11}+143 d^{10}+442 d^{9}+\cdots\right. \\
& \left.+50434 d^{4}-59080 d^{3}-40415 d^{2}+287430 d-296280\right)
\end{aligned}
$$

\footnotetext{
${ }^{2}$ Точками обозначены члены, которые мы опустили для экономии места. Полньй список полученных формул можно найти в [28], где приведены также выражения для степеней соответствующих циклов мультиособенностей в случае, когда они имеют положительную размерность.
} 


\section{Мультикасательные гиперплоскости к гиперповерхности в $\mathbb{C} P^{5}$}

$$
\begin{aligned}
\# A_{5}= & 6 d(d-2)\left(746 d^{3}-5012 d^{2}+11207 d-8340\right) \\
\# D_{5}= & 140 d(4 d-9)(d-2)^{3} \\
\# A_{1} A_{4}= & 4 d(d-2)\left(114 d^{8}-1068 d^{7}+4173 d^{6}-8835 d^{5}+10980 d^{4}\right. \\
& \left.-16324 d^{3}+57313 d^{2}-118138 d+85140\right) \\
\# A_{1} D_{4}= & 35 d(d-2)^{3}\left(d^{6}-5 d^{5}+10 d^{4}-10 d^{3}+5 d^{2}-69 d+144\right) \\
\# A_{2} A_{3}= & 18 d(d-2)\left(17 d^{8}-155 d^{7}+592 d^{6}-1230 d^{5}+1505 d^{4}-3091 d^{3}\right. \\
& \left.+13376 d^{2}-27964 d+20040\right) \\
\# A_{1}^{2} A_{3}= & \frac{3}{2} d(d-2)\left(17 d^{13}-206 d^{12}+1125 d^{11}-3660 d^{10}+7890 d^{9}+\cdots\right. \\
& \left.-196245 d^{4}+233696 d^{3}-643864 d^{2}+1256408 d-882000\right) \\
\# A_{1} A_{2}^{2}= & 18 d(d-2)\left(d^{13}-12 d^{12}+65 d^{11}-210 d^{10}+450 d^{9}-818 d^{8}+\cdots\right. \\
& \left.-12940 d^{4}+16853 d^{3}-51677 d^{2}+102274 d-71820\right) \\
\# A_{1}^{3} A_{2}= & d(d-2)\left(d^{18}-15 d^{17}+105 d^{16}-455 d^{15}+1365 d^{14}-3168 d^{13}+\cdots\right. \\
& \left.-1129608 d^{3}+2730828 d^{2}-5141832 d+3548160\right) \\
\# A_{1}^{5}= & \frac{1}{120} d(d-2)\left(d^{23}-18 d^{22}+154 d^{21}-832 d^{20}+3181 d^{19}-9332 d^{18}+\cdots\right. \\
& \left.+73475394 d^{3}-156527928 d^{2}+284455368 d-193415040\right)
\end{aligned}
$$

\section{Мультикасательные гиперплоскости к гиперповерхности в $\mathbb{C} P^{6}$}

$$
\begin{aligned}
\# A_{6}= & 7 d(d-2)\left(15926 d^{4}-142734 d^{3}+479213 d^{2}-714314 d+398856\right) \\
\# D_{6}= & 224 d(d-2)^{3}\left(46 d^{2}-209 d+237\right) \\
\# E_{6}= & 42 d(d-2)^{3}\left(97 d^{2}-426 d+468\right) \\
\# A_{1} A_{5}= & 7 d(d-2)\left(1240 d^{10}-15630 d^{9}+85751 d^{8}-268904 d^{7}+531693 d^{6}+\cdots\right. \\
& \left.+881076 d^{3}-3841807 d^{2}+5752566 d-3162744\right) \\
\# A_{1} D_{5}= & 56 d(d-2)^{3}\left(19 d^{8}-156 d^{7}+537 d^{6}-1010 d^{5}+1125 d^{4}-744 d^{3}\right. \\
& \left.-1617 d^{2}+8182 d-8952\right) \\
\# A_{2} A_{4}= & 7 d(d-2)\left(742 d^{10}-9135 d^{9}+49070 d^{8}-151025 d^{7}+293748 d^{6}+\cdots\right. \\
& \left.+1132215 d^{3}-4197325 d^{2}+6167502 d-3364920\right) \\
\# A_{2} D_{4}= & 56 d(d-2)^{3}\left(7 d^{8}-56 d^{7}+189 d^{6}-350 d^{5}+385 d^{4}-252 d^{3}+\cdots\right. \\
& \left.-1205 d^{2}+5626 d-6144\right) \\
\# A_{3}^{2}= & \frac{7}{2} d(d-2)\left(700 d^{10}-8540 d^{9}+45507 d^{8}-139076 d^{7}+268849 d^{6}+\cdots\right. \\
& \left.+1299760 d^{3}-4672757 d^{2}+6818290 d-3701976\right)
\end{aligned}
$$




$$
\begin{aligned}
\# A_{1}^{2} A_{4}= & \frac{7}{2} d(d-2)\left(106 d^{16}-1729 d^{15}+12972 d^{14}-59386 d^{13}+185482 d^{12}+\cdots\right. \\
& \left.-6493787 d^{3}+35146830 d^{2}-52506056 d+28436160\right) \\
\# A_{1}^{2} D_{4}= & 28 d(d-2)^{3}\left(d^{14}-12 d^{13}+66 d^{12}-220 d^{11}+495 d^{10}-792 d^{9}+\cdots\right. \\
& \left.+9090 d^{5}-10224 d^{4}+6750 d^{3}+8471 d^{2}-46098 d+49500\right) \\
\# A_{1} A_{2} A_{3}= & 7 d(d-2)\left(70 d^{16}-1127 d^{15}+8358 d^{14}-37870 d^{13}+117194 d^{12}+\cdots\right. \\
& \left.-8489681 d^{3}+38495186 d^{2}-56595816 d+30458304\right) \\
\# A_{2}^{3}= & \frac{7}{6} d(d-2)\left(49 d^{16}-784 d^{15}+5782 d^{14}-26068 d^{13}+80311 d^{12}+\cdots\right. \\
& \left.-9304861 d^{3}+37829206 d^{2}-55148904 d+29638080\right) \\
\# A_{1}^{3} A_{3}= & \frac{7}{6} d(d-2)\left(10 d^{22}-201 d^{21}+1908 d^{20}-11373 d^{19}+47736 d^{18}+\cdots\right. \\
& \left.+56118029 d^{3}-358281042 d^{2}+532314216 d-284437440\right) \\
\# A_{1}^{2} A_{2}^{2}= & \frac{7}{4} d(d-2)\left(7 d^{22}-140 d^{21}+1323 d^{20}-7854 d^{19}+32844 d^{18}+\cdots\right. \\
& \left.+64936185 d^{3}-356391246 d^{2}+525011976 d-280089792\right) \\
\# A_{1}^{4} A_{2}= & \frac{7}{24} d(d-2)\left(d^{28}-24 d^{27}+276 d^{26}-2024 d^{25}+10626 d^{24}+\cdots\right. \\
& \left.-494311612 d^{3}+3664055868 d^{2}-5430061008 d+2876929920\right) \\
\# A_{1}^{6}= & \frac{1}{720} d(d-2)\left(d^{34}-28 d^{33}+379 d^{32}-3302 d^{31}+20801 d^{30}+\cdots\right. \\
& \left.-287028053004 d^{2}+426393893520 d-224430998400\right)
\end{aligned}
$$

Формулы для прямых на плоскости и плоскостей в трехмерном пространстве - классические результаты Плюккера и Сальмона. По-видимому, подобные формулы для гиперповерхностей в $\mathbb{C} P^{n}, n \geqslant 4$, не были известны ранее. Простейший случай - 495 гиперплоскостей в $\mathbb{C} P^{4}$, касающихся в 4 точках заданную гиперповерхность степени 3 (число \# $A_{1}^{4}$ из соответствующей таблицы при $d=3$ ).

Исчисление касаний проективных гиперповерхностей с проективными подпространствами. Очень близкая задача состоит в следующем. Рассмотрим гладкую проективную гиперповерхность $V \subset \mathbb{C} P^{n}$ степени $d$. Развитая вьше теория позволяет вычислить число $k$-мерных проективных подпространств $K$, имеюших заданньй набор особенностей пересечения $K \cap V$. Лежандровым многообразием в этой задаче служит $M=G_{k}(T V)$, грассманово расслоение над $V$, образованное $k$-мерными касательньми подпространствами, а базой лежандрова расслоения грассманиан $N=G_{k+1, n+1}$ всех $k$-мерных проективных подпространств в $\mathbb{C} P^{n}$. Отображение $f: M \rightarrow N$ состоит в забывании точки касания $k$-мерного проективного пространства с поверхностью $V$. Ассоциированные лежандровы характеристические классы на $M$ и гомоморфизм Гизина $f_{*}: H^{*}(M) \rightarrow H^{*}(N)$ легко вычисляются при помоши разнообразных формул присоединения аналогично случаю, рассмотренному в предыдущем пункте. Некоторые из результатов этих вычислений приведены ниже ${ }^{3}$.

\footnotetext{
${ }^{3}$ См. сноску 2 .
} 
$k=1, n=3$ (прямые в пространстве)

$$
\begin{aligned}
\# A_{4} & =5 d(7 d-12)(d-4) \\
\# A_{1} A_{3} & =2 d(d-5)(3 d-5)(d-4)(d+6) \\
\# A_{2}^{2} & =\frac{1}{2} d(d-5)(d-4)\left(d^{3}+3 d^{2}+29 d-60\right) \\
\# A_{1}^{2} A_{2} & =\frac{1}{2} d(d-6)(d-5)(d-4)\left(d^{3}+9 d^{2}+20 d-60\right) \\
\# A_{1}^{4} & =\frac{1}{12} d(d-7)(d-6)(d-5)(d-4)\left(d^{3}+6 d^{2}+7 d-30\right)
\end{aligned}
$$$$
k=1, n=4\left(\text { прямые в } \mathbb{C} P^{4}\right)
$$

$$
\begin{aligned}
& \# A_{6}=35 d(7 d-12)(3 d-10)(d-6) \\
& \# A_{1} A_{5}=5 d(d-7)(d-6)\left(17 d^{3}+69 d^{2}-662 d+840\right) \\
& \# A_{2} A_{4}=5 d(d-7)(d-6)\left(2 d^{4}+6 d^{3}+91 d^{2}-678 d+840\right) \\
& \# A_{3}^{2}=\frac{1}{2} d(d-7)(d-6)\left(d^{5}+d^{4}+65 d^{3}+395 d^{2}-3350 d+4200\right) \\
& \# A_{1}^{2} A_{4}=\frac{5}{2} d(d-8)(d-7)(d-6)\left(2 d^{4}+23 d^{3}+13 d^{2}-598 d+840\right) \\
& \# A_{1} A_{2} A_{3}=d(d-8)(d-7)(d-6)\left(d^{5}+11 d^{4}+95 d^{3}+115 d^{2}-3030 d+4200\right) \\
& \# A_{2}^{3}=\frac{1}{6} d(d-8)(d-7)(d-6)\left(d^{5}+21 d^{4}+40 d^{3}+225 d^{2}-3110 d+4200\right) \\
& \# A_{1}^{3} A_{3}=\frac{1}{6} d(d-9)(d-8)(d-7)(d-6)\left(d^{5}+21 d^{4}+125 d^{3}-165 d^{2}\right. \\
& -2710 d+4200) \\
& \# A_{1}^{2} A_{2}^{2}=\frac{1}{4} d(d-9)(d-8)(d-7)(d-6)\left(2 d^{5}+22 d^{4}+105 d^{3}\right. \\
& \left.-115 d^{2}-2750 d+4200\right) \\
& \# A_{1}^{4} A_{2}=\frac{1}{24} d(d-10)(d-9)(d-8)(d-7)(d-6)\left(3 d^{5}+33 d^{4}+115 d^{3}\right. \\
& \left.-345 d^{2}-2470 d+4200\right) \\
& \# A_{1}^{6}=\frac{1}{144} d(d-11)(d-10)(d-9)(d-8)(d-7)(d-6)\left(d^{5}+9 d^{4}+21 d^{3}\right. \\
& \left.-105 d^{2}-446 d+840\right) \\
& k=2, n=4\left(\text { плоскости в } \mathbb{C} P^{4}\right) \\
& \# A_{6}=35 d\left(187 d^{3}-1495 d^{2}+3794 d-3072\right) \\
& \# D_{6}=10 d(d-2)\left(71 d^{2}-376 d+456\right) \\
& \# E_{6}=15 d(19 d-36)(d-3)(d-2) \\
& \# A_{1} A_{5}=5 d\left(236 d^{6}-2116 d^{5}+7092 d^{4}-22919 d^{3}+98239 d^{2}-222282 d+172656\right) \\
& \# A_{1} D_{5}=10 d(d-2)\left(15 d^{5}-89 d^{4}+181 d^{3}-917 d^{2}+3752 d-4224\right) \\
& \# A_{2} A_{4}=5 d\left(40 d^{7}-312 d^{6}+864 d^{5}-976 d^{4}-12859 d^{3}+\cdots-237618 d+184320\right)
\end{aligned}
$$




$$
\begin{aligned}
\# A_{2} D_{4}= & 10 d(d-4)(d-2)\left(d^{5}+d^{4}+d^{3}+21 d^{2}-460 d+732\right) \\
\# A_{3}^{2}= & \frac{1}{2} d\left(256 d^{7}-2336 d^{6}+8981 d^{5}-19846 d^{4}+\cdots-1317380 d+1022520\right) \\
\# A_{1}^{2} A_{4}= & 10 d(d-4)\left(10 d^{8}-56 d^{7}+117 d^{6}-950 d^{5}+\cdots-105884 d+97860\right) \\
\# A_{1}^{2} D_{4}= & \frac{5}{2} d(d-2)\left(3 d^{8}-20 d^{7}+47 d^{6}-319 d^{5}+\cdots-43272 d+46800\right) \\
\# A_{1} A_{2} A_{3}= & 2 d\left(16 d^{10}-137 d^{9}+499 d^{8}-2274 d^{7}+\cdots+5600880 d-4213440\right) \\
\# A_{2}^{3}= & \frac{4}{3} d\left(d^{11}-12 d^{10}+75 d^{9}-302 d^{8}+354 d^{7}+\cdots+1356750 d-1022400\right) \\
\# A_{1}^{3} A_{3}= & \frac{1}{6} d\left(32 d^{12}-316 d^{11}+994 d^{10}-4051 d^{9}+\cdots-107808480 d+78883200\right) \\
\# A_{1}^{2} A_{2}^{2}= & d(d-4)\left(d^{12}-4 d^{11}+5 d^{10}-110 d^{9}+\cdots+5382180 d-4849740\right) \\
\# A_{1}^{4} A_{2}= & \frac{1}{12} d(d-4)\left(2 d^{14}-10 d^{13}-18 d^{12}+\cdots-113728680 d+99802800\right) \\
\# A_{1}^{6}= & \frac{1}{144} d\left(d^{17}-12 d^{16}+24 d^{15}+155 d^{14}-405 d^{13}+1082 d^{12}+\cdots\right. \\
& \left.-415820104 d^{3}+1293514896 d^{2}-2517392160 d+1781049600\right)
\end{aligned}
$$

Эти формулы согласуются с результатами, которые получили Ле Барц [38] и Колли [14], [15] в задаче исчисления мультикасательных прямых к проективньм многообразиям, а также Вайнзенкер [53], Клейман и Пино [34], [35] в задаче исчисления плоских особых кривых.

\section{1. Заключительные замечания}

1) Преимущество подхода теории особенностей к задачам исчислительной геометрии заключается в том, что он позволяет рассматривать с единой точки зрения совершенно разные задачи. Действительно, исчисление особых кривых на поверхностях, или исчисление мультикасательных прямых к гиперповерхности, или исчисление особых гиперплоских сечений заданного проективного многообразия рассматриваются здесь как различные переформулировки одной и той же задачи исчисления лежандровых особенностей и мультиособенностей (но, скажем, за исчисление мультикасательных прямых к подмногообразию коразмерности больше 1 отвечает другая классификационная задача теории особенностей, а именно классификация особенностей полных пересечений).

2) Многообразия, расслоения, и т. д., возникаюшие в геометрических задачах, обладают различными характеристическими классами. Априорные формулы, подобные формулам этой статьи, показьвают, что окончательньй результат формулируется в терминах определенных комбинаций этих характеристических классов. Этот факт не всегда виден при геометрическом рассмотрении задачи; однако учет этого факта сушественно упрощает нахождение желаемой формулы.

3) Чтобы придать смысл формулам п. 10, мы требуем выполнения некоторых условий трансверсальности. Эти условия обязательно выполняются, если степень $d$ (входящая в качестве параметра в эти формулы) достаточно велика. Случаи малых степеней требуют более подробного изучения, подобного предпринятому в [35]. Например, 
бессмысленно подсчитьвать мультикасательные прямые к кубической поверхности, поскольку она содержит прямые целиком.

4) Даже если существование остаточных многочленов и универсальных формул для классов мультиособенностей установлены, нет никакой надежды получить в замкнутом виде формулу для серии особенностей (скажем, для классов особенностей $A_{r}$, $r=1,2, \ldots$, или для классов кратных точек $\left.\bar{m}_{r}, r=1,2, \ldots\right)$. Причина заключается в том, что полная классификация особенностей ведет себя крайне нерегулярньм образом. Вычисление этих классов (либо при помоши методов теории пересечений с использованием раздутий, схем Гильберта и остаточных пересечений, либо при помощи более простого метода Римани использования симметрий) основано на полной классификации особенностей, которая неизвестна.

5) Тем не менее, можно надеяться получить соответствуюшие формулы для серий классов особенностей, если рассматривать полученные многочлены после некоторой факторизации. Это значит, что желаемая формула может быть получена с точностью до слагаемых, которые обрашаются в нуль для данной геометрической задачи. Типичньм примером служит исчисление рациональных нодальных кривых на поверхностях, см. результаты Концевича-Манина [36] и Браена-Леунга [7]-[9] (обрашение в нуль старших классов Чженя $c_{i}, i>2$, для поверхности убивает большую часть кольца лежандровых характеристических классов). Результаты Клеймана [30], [31], Каца [21], Колли [14] и автора [26] об отображениях, допускаюших лишш особенности коранга 1, служат еше одним примером. Рассуждения, используюшиеся для нахождения таких формул, зависят в сильной мере от специфики задачи. Тем не менее, даже в этом случае знание сушествования универсальной формулы может оказаться чрезвычайно полезным.

\section{СПИСОК ЛИТЕРАТУРЫ}

[1] Y. Ando. On the higher Thom polynomials of Morin singularities // Publ. Res. Inst. Math. Sci. 1987. V. 23. № 1. P. 195-207.

[2] Y. Ando. On Thom polynomials of the singularities $D_{k}$ and $E_{k} / /$ J. Math. Soc. Japan. 1996. V. 48. № 3. Р. 593-606.

[3] В.И. Арнольд. Лагранжевы и лежандровы кобордизмы // УМН. 1982. Т. 37. №2. C. 179.

[4] В.И. Арнольд. Пространство функций с умеренными особенностями // Функц. анализ и его прил. 1989. Т. 23. № 3. С. 1-10.

[5] В.И. Арнольд, А.Б. Гивенталь. Симплектическая геометрия // Итоги науки и техники. Совр. пробл. матем., Фунд. напр. Т. 4. Динамические системы - 4. М.: ВИНИТИ, 1985. C. $7-139$.

[6] В.И. Арнольд, В. В. Горюнов, О.В. Ляшко, В. А. Васильев. Особенности. І. Локальная и глобальная теория. Итоги науки и техники. Совр. пробл. матем. Фунд. напр. Т. 6. Динамические системы - 6. М.: ВИНИТИ, 1985.

[7] J. Bryan, C. Leung. The enumerative geometry of $K 3$ surfaces and modular forms // alg-geom/9711031, J. Amer. Math. Soc. 2000. V. 13. № 2. P. 371-410.

[8] J. Bryan, C. Leung. Generating functions for the number of curves on Abelian surfaces // Duke Math. J. 1999. V. 99. №2. P. 311-28; math.AG/9802125.

[9] J. Bryan, C. Leung. Counting curves on irrational surfaces // Surv. Differ. Geom. 1999. V. 5. P. 313-39.

[10] В. М. Бухштабер. Характер Чженя-Дольда в кобордизмах // Матем. сб. 1970. Т. 83. № 4. C. $576-595$. 
[11] P. J. Cameron. Combinatorics: Topics, Techniques, Algorithms. Cambridge: Cambridge Univ. Press, 1994.

[12] L. Caporaso, J. Harris. Counting plane curves of any genus // Invent. Math. 1998. V. 131. № 2. P. 345-392.

[13] D.S. Chess. A note on classes $\left[S_{1}^{k}(f)\right]$ // Singularities, Part 1 (Arcata, CA, 1981). Providence, RI: Amer. Mat. Soc., 1983. P. 221-224. (Proc. Sympos. Pure Math. V. 40.)

[14] S. J. Colley. Multiple-point formulas and line complexes // Lecture Notes in Math. 1988. V. 1311. P. 9-22.

[15] S. J. Colley. Lines having specified contact with projective varieties // Proceedings of the 1984 Vancouver Conference in Algebraic Geometry / ed. J. Carrell, A. V. Geramita, P. Russell. P. 47-70. (CMS Conf. Proc. V. 6.)

[16] W. Fulton. Intersection Theory. Berlin: Springer-Verlag, 1984. (Ergeb. Math. Grenzgeb. (3). V. 2.)

[17] T. Gaffney. The Thom polynomial of $\overline{\Sigma^{1111}} / /$ Singularities, Part 1 (Arcata, CA, 1981). Providence, RI: Amer. Mat. Soc., 1983. P. 399-408. (Proc. Sympos. Pure Math. V. 40.)

[18] L. Göttsche. A conjectural generating function for numbers of curves on surfaces // Comm. Math. Phys. 1998. V. 196. № 3. P. 523-533.

[19] A Haefliger, A. Kosiński. Un théorème de Thom sur les singularités des applications différentiables // Séminaire Henri Cartan; 9e anné: 1956/57. Quelques questions de topologie. Exp. № 8, 1958.

[20] J. Harris, L. Tu. Chern numbers of kernel and cokernel bundles // Invent. Math. 1984. V. 75. №3. P. 467-475.

[21] S. Katz. Iteration of multiple point formulas and applications to conics // Lecture Notes in Math. 1988. V. 1311. P. 147-155.

[22] М. Э. Казарян. Характеристические классы лагранжевых и лежандровых особенностей // УМH. 1995. Т. 50. № 4. C. 45-70.

[23] M. Kazarian. Characteristic classes of singularity theory // The Arnold-Gelfand Mathematical Seminars. Boston: Birkhäuser, 1997. P. 325-340.

[24] M. Kazarian Classifying spaces of singularities and Thom polynomials // New Developments in Singularity Theory (Cambridge 2000). Dordrecht: Kluwer, 2001. P. 117-134. (NATO Sci. Ser. II Math. Phys. Chem. V. 21.)

[25] M. Kazarian. Thom polynomials for Lagrange, Legendre, and critical point function singularities // Proc. London Math. Soc. (3). 2003. V. 86. Part 3. P. 707-734.

[26] М.Э. Казарян. Особенности коранга 1 и их характеристические классы (в печати).

[27] М. Э. Казарян. Локализованные многочлены Тома мультиособенностей полных пересечений (в печати).

[28] Персональная страница автора http://www.mi.ras.ru/ kazarian.

[29] С. Л. Клейман. Численная теория особенностей // УМН. 1980. Т. 35. №6. С. 69-148.

[30] S. L. Kleiman. Multiple-point formulas. I: Iteration // Acta Math. 1981. V. 147. № 2. P. $13-49$.

[31] S. L. Kleiman. Multiple-point formulas. II: The Hilbert scheme // Enumerative Geometry (Sitges, 1987). Berlin: Springer-Verlag, 1990. P. 101-138. (Lecture Notes in Math. V. 1436.)

[32] S.L. Kleiman. Intersection theory and enumerative geometry: a decade in review. With the collaboration of Anders Thorup on Sect. 3. // Algebraic Geometry (Brunswick/Maine, 1985). Providence, RI: Amer. Math. Soc., 1987. P. 321-370. (Proc. Sympos. Pure Math., 46, Part 2.)

[33] S. L. Kleiman, J. Lipman, B. Ulrich. The multiple-point schemes of a finite curvilinear map of codimension one // Ark. Mat. 1996. V. 34. № 2. P. 285-326.

[34] S. L. Kleiman, R. Piene. Enumerating singular curves on surfaces // Algebraic Geometry. Hirzebruch 70 / ed. P. Pragacz. Providence, RI: Amer. Math. Soc., 1999. P. 209-238. (Contemp. Math. V. 241.) Corrections: math.AG/9903192

[35] S.L. Kleiman, R. Piene. Node polynomials for families: results and examples // math. AG/0111299. 
[36] M. Kontsevich, Yu. Manin. Gromov-Witten classes, quantum cohomology, and enumerative geometry // Comm. Math. Phys. 1994. V. 164. № 3. P. 525-562.

[37] P.S. Landweber. Cobordism operations and Hopf algebras // Trans. Amer. Math. Soc. 1967. V. 129. № 1. P. 94-110.

[38] P. Le Barz. Géométrie énumérative pour les multisécantes // Lecture Notes in Math. 1978. V. 683. P. $116-167$.

[39] J. Mather. Stability of $C^{\infty}$-mappings. I-VI // Ann. of Math. (2). 1968. V. 87. P. 89-104; 1969. V. 89. P. 254-291; // Inst. Hautes Études Sci. Publ. 1968. V. 35. P. 279-308; 1969. V. 37. P. 223-248; // Adv. Math. 1970. V. 4. P. 301-336; // Lecture Notes in Math. 1971. V. 192. P. 207-253.

[40] Дж. Мэй. Геометрия итерированных пространств петель // Дополнение в кн.: Дж. Бордман, Р. Фогт. Гомотопические инвариантные алгебраические структуры на топологических пространствах. М.: Мир, 1977. С. 267-392.

[41] С. П. Новиков. Теорема Картана-Серра и внутренние гомологии // УМН. 1966. Т. 21. № 5. C. $217-232$.

[42] С. П. Новиков. Методы алгебраической топологии с точки зрения теории кобордизмов // Изв. АН СССР. Сер. матем. 1967. Т. 31. № 4. С. 855-951.

[43] I. R. Porteous. Simple singularities of maps // Proceedings of Liverpool Singularity Symposium I / ed. C. T. C. Wall. New York: Springer-Verlag, 1971. P. 286-307. (Lecture Notes in Math. V. 192.)

[44] I. R. Porteous. The second-order decomposition in $\Sigma^{2} / /$ Topology. 1972. V. 11. № 4. P. $325-334$

[45] D. C. Ravenel, W.S. Wilson. The Hopf ring for complex cobordism // Bull. Amer. Math. Soc. 1974. V. 80. №6. P. 1185-1189.

[46] R. Rimányi. Thom polynomials, symmetries and incidences of singularities // Invent. Math. 2001. V. 143. № 3. P. 499-521.

[47] R. Rimányi. Multiple point formulas-a new point of view // Pacific J. Math. 2002. V. 202. № 2. P. 475-490.

[48] R. Rimányi, A. Szücs. Pontrjagin-Thom-type construction for maps with singularities // Topology. 1998. V. 37. №6. P. 1177-1191.

[49] F. Ronga. Le calcul des classes duales aux singularités de Boardman d'ordre deux // Comment. Math. Helv. 1972. V. 47. № 1. P. 15-35.

[50] F. Ronga. On the number of planes triply tangent to a surface in projective space // Proceedings of the 1984 Vancouver Conference in Algebraic Geometry, July 1984 / ed. J. Carrell, A. V. Geramita, P. Russell. Providence, RI: Amer. Math. Soc., 1986. P. 389-397. (CMS Conf. Proc. V. 6.)

[51] Р. Том. Некоторые свойства "в целом" дифференцируемых многообразий // Расслоенные пространства и их приложения. М.: ИЛ, 1958. С. 293-351.

[52] R. Thom. Les singularités des applications différentiables // Ann. Inst. Fourier (Grenoble). 1955-1956. V. 6. P. 43-87.

[53] I. Vainsencher. Enumeration of $n$-fold tangent hyperplanes to a surface // J. Algebraic Geom. 1995. V. 4. № 3. P. 503-526.

[54] В. А. Васильев. Лагранжевы и лежандровы характеристические классы. М.: МЦНМО, 2000.

[55] В. А. Васильев. Топология дополнения к дискриминантам. М.: Фазис, 1997.

[56] C. T. C. Wall. A second note on symmetry of singularities // Bull. London Math. Soc. 1980. V. 12. № 5. P. 347-354. 